\title{
ANÁLISE DE EXPERIMENTOS EM PARCELAS SUBDIVIDI- DAS COM TRATAMENTOS PRINCIPAIS DISPOSTOS EM BLOCOS INCOMPLETOS BALANCEADOS
}

\author{
ANTONIO FRANCISCO IEMMA
}

Orientador: Prof. Dr. HUMBERTO DE CAMPOS

Tese apresentada à Escola Superior de Agricultura "Luiz de Queiroz", da Universidade de São Paulo, para obtenção do título de Doutor em Agronomia. Área de concentração: Estatística e Experimentação Agronômica.

PIRACICABA

Estado de São Paulo, Brasil

Abril, 1981 
ii.

A Deus,

OFEREÇO.

As minhas quatro Anas,

aos meus pais

$e$ ao meu irmão,

DEDICO. 


\section{AGRADECIMENTOS}

Ao Dr. Humberto de Campos, Professor Adjunto do Departamento de Ma temática e Estatística da ESALQ/USP, pela orientação segura e amiga na execução deste trabalho.

Aos Professores do Curso de Pös-Graduação em Estatística e Experimentação Agronômica da ESALQ/USP, na pessoa de seu Coordenador Prof. Humberto de Campos, por sua acolhida, seus ensinamentos e pela solicitude com a qual invariavelmente nos atenderam dentro e fora das salas de aula.

Aos Professores Carlos Roberto Padovani, Clarice Garcia Borges Demé trio, Décio Barbin, Frederico Pimentel Gomes, Paulo Roberto Curi, Ubirajara Dorival Diniz e Waldenir Queiroz Ribeiro, pelas va liosas sugestões.

Aos Professores Rui Vieira de Morais e Sheila Zambello de Pinho, pelo auxílio na execução dos programas para o computador IBM 1130 da UNESP - Campus de Botucatu, utilizados neste estudo.

Aos Professores do Departamento de Bioestatística, à Diretoria do Instituto Básico de Biologia Médica e Agrícola do Campus de Botucatu, à Reitoria da UNESP, bem como aos seus respectivos órgãos colegiados, pelo afastamento concedido.

A CAPES - Coordenação do Aperfeiçoamento do Pessoal do Nível Superior, pela bolsa de estudos concedida.

Ao Prof. Antonio Joaquim Rocha, ao Dr. Antonio Carlos Vilella Braga e ao Dr. Electro Bonini, pela confiança depositada em nossos primeiros passos. 
Ȧs Srtas. Maria Izalina Ferreira Alves e Rosa Maria Alves, bem como ao Sr. Octávio Frassetto, pela datilografia e impressão.

A Eng. Agrå. Eliza Helena de Souza Faria, pela revisão final do tra balho.

Aos companheiros do Curso de Pós-Graduação em Estatística e Experimentação Agronômica, pelo convívio.

Aos meus amigos Abraão Adolpho Angeli, Humberto Spinetti Jr., José Paulo Peter Lacorte e José Roberto Braga de Camargo,bem como às respectivas famílias, pelo apoio.

A minha família, pela paciência e dedicação.

A todos que direta ou indiretamente contribuiram na execução deste trabalho. 
Pàg.

RESUMO

$\mathbf{X}$

SUMMARY xili

1. INTRODUÇÃO

1.1 - Localização do Problema $\ldots \ldots \ldots \ldots \ldots \ldots \ldots \ldots \ldots \ldots$

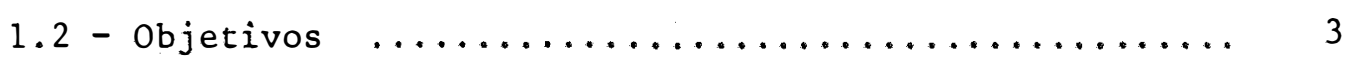

2. REVISÃO BIBLIOGRฐFICA $\ldots \ldots \ldots \ldots \ldots \ldots \ldots \ldots \ldots \ldots$

2.1 - Ensaios em Parcelas Subdivididas ............. 4

2.1 .1 - Generalidades $\ldots \ldots \ldots \ldots \ldots \ldots \ldots \ldots \ldots . \ldots . \ldots 4$

2.1 .2 - Natureza do erro experimental ......... 7

2.1 .3 - Funções lineares estimáveis e suas variâncias 8

2.1 .4 - Anālise de variância $\ldots \ldots \ldots \ldots \ldots \ldots . . \ldots 11$

2.2 - Ensaios em Blocos Incompletos Balanceados ....... 14

2.2.1 - Generalidades ....................... 14

2.2.2 - Matriz de variâncias e covariâncias ...... 16

2.2 .3 - Variância da função linear estimável ..... 17

2.2 .4 - Anālise de variância $\ldots \ldots \ldots \ldots \ldots \ldots \ldots .618$

2.3 - Ensaios com Parcelas Subdivididas em Blocos Incomple tos .................................... 19

2.3.1 - Generalidades $\ldots \ldots \ldots \ldots \ldots \ldots \ldots \ldots . .6 . \ldots$

2.3 .2 - o erro experimental ............... 21

2.3 .3 - Funções lineares estimáveis e suas variâncias 23

2.3 .4 - Anālise de variância ................. 25 
Pảg.

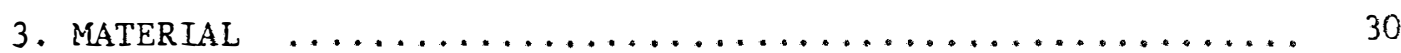

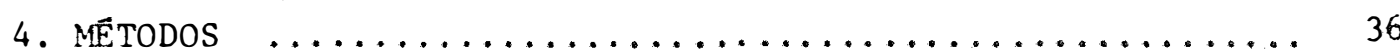

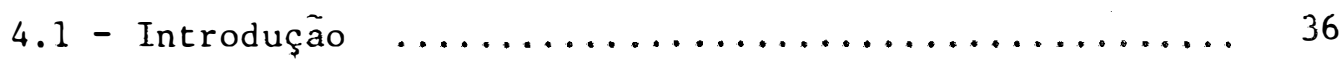

4.2 - Modelo Matemätico ..................... 39

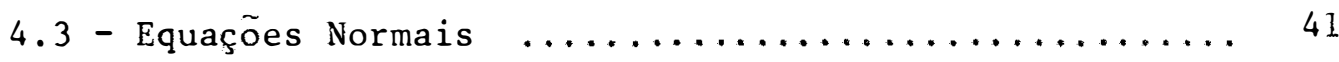

4.4 - Solução do Sistema ................... 49

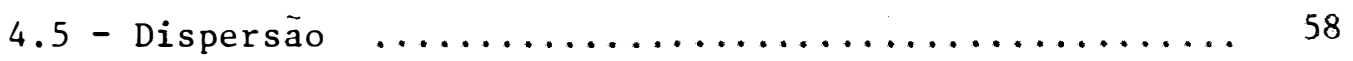

4.5 .1 - Dispersão para tratamentos principais .... 60

4.5 .2 - Dispersão para tratamentos secundärios ... 66

4.5 .3 - Dispersão para a interação :... $^{\prime} \ldots . . \ldots .668$

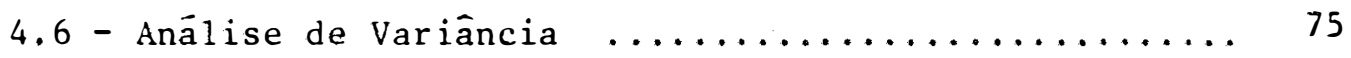

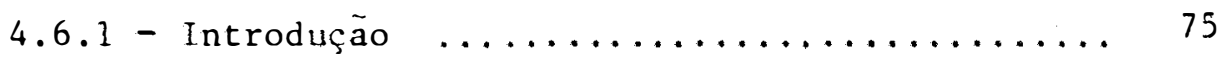

4.6.2 - Esperanças dos quadrados médios para os efei tos fixos ..................... 79

4.6.2.1 - Soma de quadrados dos parâmetros.. 79

4.6.2.2 - Somas de quadrados por parâmetro.. 83

4.6.2.2.1 - Média geral ...... 83

4.6.2.2.2 - Tratamentos principais 87

$4.6 .2 .2 .3-$ Blocos $\ldots \ldots \ldots \ldots .69$

4.6.2.2.4 - Tratamentos secundārios 91

4.6 .2 .2 .5 - Interação $t \times t^{\prime} \ldots . .99$

4.6 .3 - Soma de quadrados devida às parcelas ..... 98 
vii.

2å.

4.6 .4 - Soma de quadrados devida ao total ....... 100

4.6.5 - Componentes de variância devidos aos efeitos aleatórios ....................... 102

4.6 .5 .1 - Resíduo (a) $\ldots \ldots \ldots \ldots \ldots . \ldots 102$

4.6 .5 .2 - Resíduo (b) $\ldots \ldots \ldots \ldots \ldots \ldots \ldots 2$

4.6.6 - Testes de hipóteses e quadros de análise .. 103

4.6 .7 - Comparações mültiplas ............ 114

4.6.7.1 - Entre tratamentos principais ... 115

4.6.7.2 - Entre tratamentos secundārios ... 115

4.6.7.3 - Entre tratamentos secundārios dentro do i-ésimo tratamento principal $\ldots \ldots \ldots \ldots \ldots \ldots \ldots \ldots \ldots \ldots$

4.6.7.4 - Entre tratamentos principais dentro do s-ésimo tratamento secundārio

4.7 - Testes Sobre a Estrutura da Matriz de Covariâncias..

4.7.1 - Teste de homogeneidade das matrizes de covariâncias para todos os tratamentos ...... 118

4.7.2 - Teste de uniformidade da matriz comum de covariâncias ....................... 120

5. ILUSTRAÇÃO DO METODO PROPOSTO $\ldots \ldots \ldots \ldots \ldots \ldots \ldots \ldots \ldots \ldots . \ldots . \ldots . \ldots 22$

5.1 - Testes Sobre a Estrutura da Matriz de Covariân-

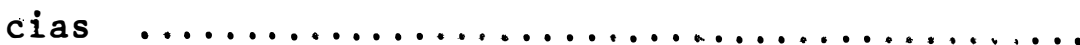


viii.

Pàg.

5.1.1 - Teste de homogeneidade das matrizes de covariâncias $\ldots \ldots \ldots \ldots \ldots \ldots \ldots . \ldots \ldots \ldots$

5.1 .2 - Teste de uniformidade da matriz comum de covariâncias $\ldots \ldots \ldots \ldots \ldots \ldots \ldots \ldots \ldots$

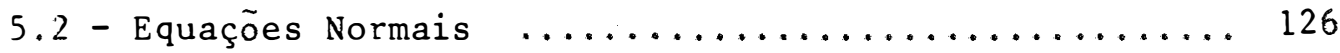

5.3 - Solução do Sistema $\ldots \ldots \ldots \ldots \ldots \ldots \ldots \ldots \ldots \ldots \ldots \ldots$

5.3.1 - Efeitos estimados de tratamentos principais 130

5.3.2 - Efeitos estimados de tratamentos secundários 130

5.3 .3 - Efeitos estimados dos pares $\left(t t^{\prime}\right)$ is $\ldots . . .131$

5.4 - Anālise de Variância ................... 132

5.4 .1 - Obtenção das somas de quadrados ........ 132

5.4 .2 - Testes de hipōteses e quadros de análise .. 133

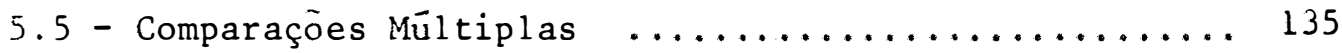

5.5.1 - Entre médias de efeitos estimados de tratamentos principais ............... 135

5.5.2 - Entre médias de efeitos estimados de tratamentos secundārios ................. 135

5.5.3 - Entre médias de efeitos estimados de tratamentos secundários dentro do i-ésimo tratamento principal ................ 136

5.5.4 - Entre médias de efeitos estimados de tratamentos principais, dentro do s-ésimo trata-

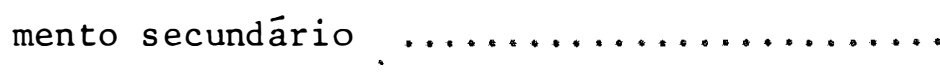


pàg.

6. CONCLUSŎES $\ldots \ldots \ldots \ldots \ldots \ldots \ldots \ldots \ldots \ldots \ldots \ldots \ldots \ldots \ldots \ldots$

6.1 - Em Relação ao Modelo Matemático .............. 138

6.2 - Em Relaçào ao Sistema de Equações Normais ....... 139

6.3 - Em Relação às Variâncias das Funções Lineares Esti-

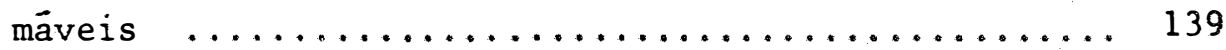

6.4 - Em Relação à Análise de Variância ............. 140

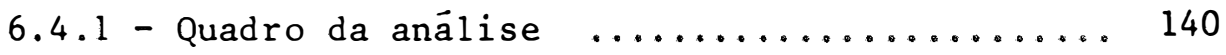

6.4 .2 - Testes de hipöteses ................ 140

6.5 - Em Relação às Comparações Múltiplas ........... 141

7. REFERENCIAS BIBL IOGRAFICAS $\ldots \ldots \ldots \ldots \ldots \ldots \ldots \ldots \ldots \ldots \ldots$ 


\section{ANAL ISE DE EXPERIMENTOS EM PARCELAS SUBDIVIDIDAS COM TRATAMENTOS PRINCIPAIS DISPOSTOS EM BLOCOS INCOMPLETOS BALANCEADOS}

$\begin{array}{ll}\text { Autor: } & \text { Antonio Francisco Iemma } \\ \text { Orientador: } & \text { Prof. Dr. Humberto de Campos }\end{array}$

RESUMO

Neste estudo, analisou-se o comportamento dos experimentos em parcelas subdivididas com tratamentos principais dispostos em blocos incompletos balanceados.

Para tanto, adotou-se o modelo matemático:

$$
y_{i j s}=m+t_{i}+b_{j}+e_{k(i j)}+t_{s}^{\prime}+\left(t t^{\prime}\right)_{i s}+e_{i j s}
$$

onde, para $i=1,2, \ldots, v ; j=1,2, \ldots, a ; s=1,2, \ldots, u ; k=1,2, \ldots, k$,

$y_{i j s}$ é o valor observado na subparcela que recebeu o s-ésimo tratamento secundário, dentro do i-ésimo tratamento prin cipal, no bloco $\underline{j}$;

m è o efeito da média geral;

$t_{i}$ è o efeito do i-ésimo tratamento principal;

$b_{j}$ è o efeito do j-ésimo bloco; 
$e_{k(i j)}$ é o erro atribuỉdo à k-ésima parcela do bloco $\underline{j}_{\text {, que }}$ recebeu o tratamento principal $\underline{i}$ : caracterizado como componente do erro (a);

$t_{s}^{\prime}$ é o efeito do s-ésimo tratamento secundärio;

$\left(t t^{\prime}\right)$ is é o efeito da interação entre o i-ésimo tratamento prin cipal e o s-ésimo tratamento secundārio;

$e_{i j s}$ é o erro atribuỉdo à observação y $y_{i j s}$, caracterizado co mo componente do erro (b).

Ademais, considerou-se a existência de correlação constante, $p$, entre duas subparcelas de uma mesma parcela, e independência entre subparcelas de parcelas distintas, resultando

$$
\operatorname{Cov}\left(y_{i j s^{\prime}} y_{i^{\prime} j^{\prime} s^{\prime}}\right)= \begin{cases}\sigma^{2}, & \text { se } i=i^{\prime}, j=j^{\prime}, s=s^{\prime} \\ \rho \sigma^{2}, & \text { se } i=i^{\prime}, j=j^{\prime}, s \neq s^{\prime} \\ 0, & \text { em outros casos }\end{cases}
$$

No desenvolvimento da metodologia, supôs-se um ensaio com parcelas subdivididas no qual os $\underline{v}$ tratamentos principais estivessem presentes em $\underline{r}$ dos $\underline{a}$ blocos incompletos balanceados $(r<a)$; cada bloco apresentasse $k$ parcelas e cada par de tratamentos princi pais ocorresse em $\lambda$ blocos.

Sob essas condições, foram determinados: o sistema de equações normais, as matrizes de dispersão para os parâmetros bạ sicos, os componentes de variância para os efeitos fixos e aleatórios do modelo proposto, os critérios para os testes das hipóteses 
de nulidade usuais e os critërios para comparações mültiplas pelo teste de Tukey baseados nas variâncias das funções lineares estimáveis.

A menos de constantes inerentes ao modelo, duas situações distintas ficaram caracterizadas, no tocante às determinaçôes citadas. Assim, as estimativas referentes a tratamentos princi pais e blocos, obtidas neste estudo, foram análogas àquelas encontradas na bibliografia dos ensaios em blocos incompletos balanceados; enquanto que as estimativas referente a tratamentos secundários e à interação $t \times t^{\prime}$, portaram-se de modo semelhante às suas corres pondentes em ensaios com parcelas subdivididas em blocos casualizados. 
xiii.

\section{ANALYSIS OF SPLIT-PLOT EXPERIMENTS WITH MAIN TREATMENTS DISPOSED IN BALANCED \\ INCOMPLETE BLOCK}

Author: Antonio Francisco Iemma

Adviser: Prof. Or. Humberto de Campos

SUMMARY

In this paper, the conduct of the split-plot designs with the main treatments disposed in balanced incomplete blocks, was analised.

For this, the following mathematical model, was used:

$$
y_{i j s}=m+t_{i}+b_{j}+e_{k(i j)}+t_{s}^{\prime}+\left(t t^{\prime}\right)_{i s}+e_{i j s}
$$

where, for $i=1, \ldots, v ; j=1, \ldots, a ; s=1, \ldots, u ; k=1, \ldots, k$,

$y_{i j s}$ denotes the observation of the subplot containing the $s$ th subtreatment of the $i$ th main treatment in the $j$ th block;

m denotes the effect of general mean;

$t_{i}$ denotes the effect of the $i$ th main treatment;

$b_{j}$ denotes the effect of the $j$ th block; 
xiv.

$e_{k(i j)}$ denotes the error inherent at the $k$ th whole-plot of the $j$ th block in the $i$ th mean treatment, characterized with a error (a) component;

$t_{s}^{\prime}$ denotes the effect of the $s$ th sub treatment;

$\left(t t^{\prime}\right)$ is denotes the effect for the interaction between $i$ th main treatment and $s$ th sub treatment;

$e_{i j s}$ denotes the error inherent at the $i-j-s$ th sub plot, characterized with a error (b) component.

Moreover, the presence of constant correlaction, $\rho$, between two subplots of same plot, and independence among subplots of the distincts plots, was considered, resulting:

$$
\operatorname{Cov}\left(y_{i, j s}, y_{i^{\prime} j^{\prime} s^{\prime}}\right)= \begin{cases}\sigma^{2}, & \text { if } i=i^{\prime}, j=j^{\prime}, s=s^{\prime} \\ \rho \sigma^{2}, & \text { if } i=i^{\prime}, j=j^{\prime}, s \neq s^{\prime} \\ 0, & \text { otherwise }\end{cases}
$$

In methodology one split-plot design with the $\underline{v}$ main

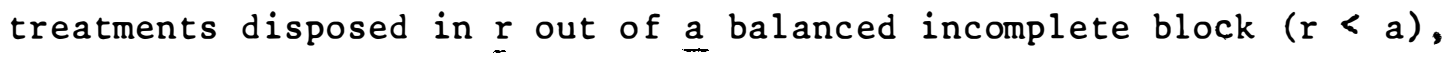
where every block was formed by $k$ plots, and every pair of main treatments occurred in $\lambda$ blocks, was considered.

Under these assumptions, the solution of the normal equations, the dispersion matrix for the effects in the mathematical model proposed, the criterion for the usual null hypothesis tests and the criterion of the multiple comparisions by Tukey's test based on 1 inear functions variance, was obtained. 
Except for constants inherents in the model, two main conclusions were obtained: estimates of main treatments and blocks, obtained in this study, were analogous to those existent in the $11-$ terature of the balanced incomplete block design; while other estimates were analogous to those correspondents in the split-plot randomized block design. 
1. INTRODUÇAOO

1.1 - Localizaçăo do Problema

Os experimentos em parcelas subdivididas, que segundo LEONARD e CLARK (1939) foram propostos por Yates, em 1933, têm si do de grande valia para os estudiosos das mais diversas áreas de pes quisa. No tocante à pesquisa agropecuária, sua aplicação vem sendo ressaltada por autores consagrados, comp STELL e TORRIE (1960), SNE DECOR e COCHRAN (1967), PIMENTEL GOMES (1976), COCHRAN e COX (1976), dentre outros.

A farta bibliografia existente em relação a tais ensaios tem mostrado uma certa tendência dos autores para a disposição dos tratamentos principais em blocos casualizados e esse fato pode, de certo modo, ser justificądo pela grande simplicidade e al- 
ta eficiéncia desses delineamentos.

No entanto, se por um lado os blocos casualizados são recomendáveis, por outro eles podem apresentar o inconveniente de fugir ao controle do experimentador, quando num ensaio em parcelas subdivididas o número de tratamentos secundärios for relativamente grande. E natural que se o efeito de blocos for significativo eles devem ser preferidos sobre um ensaio inteiramente casualizado, mesmo que o número de tratamentos secundārios seja grande, apesar do risco da crescente perda de eficiência diretamente associada ao tamanho dos blocos.

Alēm do inconveniente citado, existem outros que, na pesquisa agropecuāria, podem ser de caráter restritivo à formação dos blocos casualizados. Como ilustração, tomemos um ensaio com sui nos no qual se deseja testar um grande número de tratamentos. Considerando-se que para experimentos desse tipo está consagrado o uso de leitegadas como blocos, o experimentador tem diante de si um impasse: ou diminui o número de tratamentos ou não usa leitegada como bloco. Na primeira escolha perderá a vantagem de comparar os tratamentos eliminados com os demais, na segunda fatalmente perderá em eficiência, dada a menor homogeneidade dos "novos" blocos.

Assim como esta, existem inúmeras outras situações, na pesquisa agropecuāria, que sugerem uma nova utilização dos experimentos em parcelas subdivididas em blocos, que se torne mais geral e menos restritiva, permitindo ao experimentador uma maior abran 
gência, no tocante a suas aplicações: os experimentos em parcelas subdivididas em blocos incompletos.

\section{2 - objetivos}

No desenvolvimento da revisão bibliogräfica, notou- se uma lacuna quanto aos ensaios com parcelas subdivididas em blocos incompletos. Assim, propôs-se como um dos objetivos deste traba 1ho, apresentar-se um estudo sobre esse tema. No entanto,dado a sua extensão, optou-se pelo caso em que os tratamentos principais estivessem dispostos em blocos incompletos balanceados, ficando os casos de blocos parcialmente balanceados, bem como o estudo dos trata mentos secundärios em blocos incompletos e os casos não balanceados, para estudos posteriores.

Ademais, preocupou-se em redigir um trabalho que pudesse ser entendido e usado por profissionais da ārea agropecuária. Desse modo, apesar de adotar-se a sólida estrutura da Teoria dos Mo delos Lineares, ela foi muitas vezes, dissimulada pelo uso da termi nologia tradicional e já familiar para tais profissionais. 
2. REVISAOO BIBLIOGRAFICA

\section{1 - Ensaios em Parcelas Subdivididas}

\subsection{1 - General idades}

Segundo LEONARD e CLARK (1939), os Ensaios em Parcelas Subdivididas tiveram seu início com Yates, por volta de 1933,se guido de Le Clerg, em 1937, e Goulden em 1939.

Dentre suas mültiplas aplicações, PIMENTEL GOMES (1976) destaca o estudo de dois tipos de tratamentos, como por exem plo, variedades e esdąamentos. Nesse caso. cada Darcela semeada com uma certa variedade seria redartida em subparcelas. cada qual com um dos esdaçamentos de interesse.

COCHRAN e COX (1976). definindo os Ensaios em Parcelas Subdivididas através dos ensaios em fatorial com confundimento 
de efeitos princidais, ressaltam sua eficiência em exoerimentos de campo, auando se deseia introduzir um fator adicional dentro do ensaio. Assim, Dor exemolo, se existe o plano inicial dara testar-se um Eator A em I niveis, entăo a divisão de cada parcela, digamos, ao meio, permite a introdução de um novo fator $B$, a dois niveis que serão distribuıdos casualmente dentro de cada parcela dos níveis de A.

Por sua vez, LEAL (1979) enfoca o uso dos experimentos em parcelas subdivididas, na análise dos ensaios com medidas re petidas sobre unidades experimentais, como uma alternativa para o uso da Anälise Multivariada, quando se constata a uniformidade da matriz de variâncias e covariâncias. Sob esse prisma, concorda com STEEL e TORRIE (1960), CALZADA BENZA (1970) e LiTTLE e HILLS (1972). que arqumentam que os experimentos nos quais observações sucessivas são feitas sob uma mesma unidade experimental durante um certo intervalo de tempo, em muitos aspectos se assemelham a experimentos em parcelas subdivididas, nos quais cada unidade experimental é dividi da em subunidades distintas.

No entanto, Dara STEEL e TORRIE (1960) existem alsumas diferenças fundamentais entre as anālises desses dois tipos de experimentos. Por exemplo, na anālise de ensaios com medidas repeti das, quando o delineamento é o de blocos casualizados, a soma de qua drados da interação blocos $x$ medidas no tempo deve sempre ser separada do erro (b), dada a sua importância nas conclusões sobre o ensaio. 
Outra diferença importante, também citada em LEAL (1979), consiste na composiçào da matriz de variâncias e covariâncias, que para medials repetidas no tempo é do tipo

$$
\therefore=\left[\begin{array}{cccc}
\sigma_{11} & \sigma_{12} & \ldots & \sigma_{1 p} \\
\sigma_{12} & \sigma_{22} & \ldots & \sigma_{2 p} \\
\ldots & \ldots & \ldots & \ldots \\
\sigma_{1 p} & \sigma_{2 p} & \ldots & \sigma_{p p}
\end{array}\right]
$$

correspondendo muito mais à estrutura exigida para a análise multivariada, enquanto que, para ensaios com parcelas subdivididas, exis te a pressuposição mais restritiva que as observações têm variâncias homogêneas e sao igualmente correlacionadas, gerando uma matriz de variâncias e covariâncias do tipo uniforme. Então

$$
c_{k k^{\prime}}=\left\{\begin{array}{lll}
\sigma^{2}, & \text { se } & k=k^{\prime} \\
\rho \sigma^{2}, & \text { se } & k \neq k^{\prime}
\end{array}\right.
$$

resultando:

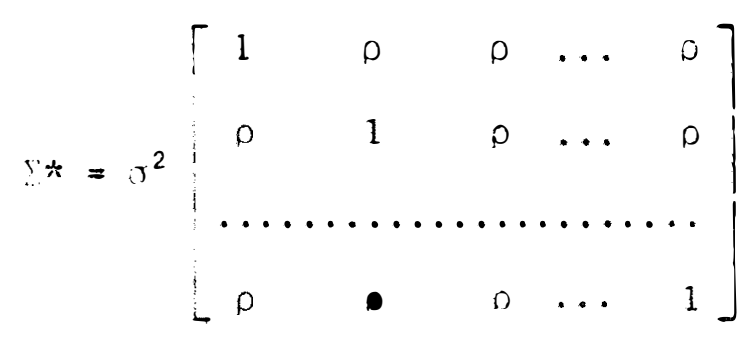

DINIZ (1980) cita Gill e Hafs (1971), ao salientar a necessidade de se verificar se as matrizes de dispersão para tratamentos são homogêneas, assim como, se a matriz de dispersão do expe 
rimento é do tipo uniforme, para que seja adequado o uso da técníca das parcelas subdivididas.

\subsection{2 - Natureza do erro experimental}

Segundo COCHRAN e COX (1976), na anālise estatística dos experimentos em parcelas subdivididas, deve ser considerado o fa to de que as observações das diferentes subparcelas de uma mesma parcela podem estar correlacionadas. Para ressaltar sua colocação exemplificam alegando que, em experimentos de campo, essa correlaçào pode ser motivada pela proximidade das plantas, que nessas condições tendem a ser similares em suas propriedades agronômicas. Assim como em CHAKRABARTI (1962), LEAL (1979) e DINIZ (1980), dentre outros, COCHRAN e COX (1976) mostram que se supõe existir uma correlação, $\rho$, constante entre erros experimentais do tipo $e_{i j k}$ e $e_{i j k}$ ', para quaisquer subparcelas de uma mesma parce la, tal que

$$
E\left(e_{i j k}\right)=0, V\left(e_{i j k}\right)=\sigma^{2}
$$

e

$$
\operatorname{Cov}\left(e_{i j k}, e_{i} j^{\prime} k^{\prime}\right)= \begin{cases}\rho \sigma^{2}, & \text { se } i=i^{\prime}, j=j^{\prime}, k=k^{\prime} \\ 0, & \text { em outros casos }\end{cases}
$$

Desse modo, exemplificam que, para duas subparcelas por parcela, a variância do erro de uma parcela total è

$$
E\left(e_{i j_{1}}+e_{i j_{2}}\right)^{2}=\sigma^{2}+\sigma^{2}+2 \rho \sigma^{2}=2(1+\rho) \sigma^{2} .
$$


Por outro lado, mostram que para o tratamento princi pal, a variância é do tipo $\left[1+(\beta-1) \rho j \sigma^{2}\right.$.

No tocante à eficiência dos testes para tratamentos principais e secundários, KEMPTHORNE (1950), TAYLOR (1950), FEDERER (1955) e PIMENTEL GOMES (1976), dentre outros, são unânimes em afirmar a maior precisão existente no teste de tratamentos secundários. Sob esse aspecto, PIMENTEL GOMES (1976) ressalta que essa diferença de eficiências pode ser usada como uma vantagem adicional.

Para COCHRAN e COX (1976) a maior precisão obtida no teste dos tratamentos secundários pode ser justificada pelo coeficí ente, $\rho$, de correlação que, na prätica, é quase sempre positivo.

Este fato pode ser melhor interpretado quando se toma a estimativa não tendenciosa para p citada em LEAL (1979)

$$
\hat{\rho}=\frac{Q M \operatorname{Res}(a)}{Q M \operatorname{Res}(a)+Q M \operatorname{Res}(b)}
$$

\subsection{3 - Funções lineares estimāveis e suas variāncias}

No tocante à variância das funções lineares estimāveis, os autores mostraram-se, de certo modo coerentes, a menos do coeficiente, $\rho$, de correlação que, para alguns autores, não é consi derado.

Assim, em CHAKRABARTI (1962) encontra-se o seguinte quadro para estimadores e suas variâncias: 


\begin{tabular}{|c|c|c|}
\hline $\begin{array}{c}\text { Funções Lineares } \\
\text { dos Parâmetros }\end{array}$ & $\begin{array}{c}\text { Melhor Estimador } \\
\text { Linear }\end{array}$ & Variâncias \\
\hline$\alpha_{i}-\alpha_{i}$ & $y_{i} . .-y_{i}, \ldots$ & $2 \sigma_{2}^{2} / \alpha \beta$ \\
\hline$\rho_{j}-\rho_{j}{ }^{\prime}$ & ${ }^{y} \cdot j \cdot{ }^{-y} \cdot j^{\prime}$ & $2 \sigma_{2}^{2} / r \beta$ \\
\hline$\tau_{k}-\tau_{k}$ & $y \ldots k^{-y} \ldots k^{\prime}$ & $2 \sigma_{1}^{2} \operatorname{lr} \alpha$ \\
\hline$\delta_{j k}-\delta_{j \prime k}$ & $\left.{ }^{y} \cdot j k^{-y} \cdot j^{\prime} k^{-(y} \cdot j \cdot{ }^{-y} \cdot j^{\prime} \cdot\right)$ & $2 \sigma_{1}^{2} / r\left(1-\frac{1}{\beta}\right)$ \\
\hline$\delta_{j k}-\delta_{j k^{\prime}}$ & $\left.{ }^{y} \cdot j k^{-y} \cdot j k^{\prime-(y} \ldots k^{-y} \ldots k^{\prime}\right)$ & $2 \sigma_{1}^{2} / r\left(1-\frac{1}{\alpha}\right)$ \\
\hline
\end{tabular}

obtido do modelo

$$
y_{i j k}=\mu+\alpha_{i}+\rho_{j}+\tau_{k}+\delta_{j k}+e_{i j k},
$$

onde, $\alpha_{i}$ denota o efeito do i-ésimo bloco;

$\rho_{j}$ denota o efeito do j-ésimo tratamento principal;

$\tau_{k}$ denota o efeito do k-ésimo tratamento secundārio;

$\delta_{j k}$ denota o efeito da interação do j-ésimo tratamento principal com o k-ésimo tratamento secundārio;

$e_{i j k}$ é o erro experimental, normalmente distribuỉdo

$i=1,2, \ldots, r$ blocos;

$j=1,2, \ldots, \alpha$ tratamentos principais;

$k=1,2, \ldots, B$ tratamentos secundários.

Ademais,

$$
\sigma_{2}^{2}=[1+(\beta-1) \rho] \sigma^{2} \quad \text { e } \quad \sigma_{1}^{2}=(1-\rho) \sigma^{2} \text {. }
$$


Por sua vez, PIMENTEL GOMES (1976) apresenta resulta dos que podem ser resumidos no seguinte quadro:

\begin{tabular}{|c|c|c|c|}
\hline $\begin{array}{l}\text { Comparação Entre Médias } \\
\text { dos Efeitos Estimados }\end{array}$ & \multicolumn{3}{|c|}{$\begin{array}{l}\text { Estimativas das } \\
\text { Variancias }\end{array}$} \\
\hline$\hat{\mathrm{m}}_{A}-\hat{\mathrm{m}}_{\mathrm{A}}$, & & $(2 / b r)$ QMRes (a) & \\
\hline$\hat{\mathrm{m}}_{B}-\hat{\mathrm{m}}_{\mathrm{B}^{\prime}}$ & & $(2 / a r) Q \operatorname{Res}(b)$ & \\
\hline$\widehat{\mathrm{m}}_{\mathrm{AB}}-\widehat{\mathrm{m}}_{\mathrm{AB}}$ & & $(2 / r) \quad$ QMRes $(b)$ & \\
\hline$\hat{\mathrm{m}}_{\mathrm{AB}}-\hat{\mathrm{m}}_{\mathrm{A}^{\prime} \mathrm{B}}$ & $(2 / \mathrm{br})$ & $Q \operatorname{Res}(a)+(b-1)$ & QMRes (b) \\
\hline
\end{tabular}

onde, $\mathrm{a}=\mathrm{n}$ ? de tratamentos principais;

$\mathrm{b}=\mathrm{n}$ ? de tratamentos secundārios;

$r=n$ o de repetições;

A e B referem-se aos tratamentos principais e secundários, res pectivamente;

e, no teste de $\hat{\mathrm{m}}_{A B}-\widehat{\mathrm{m}}_{\mathrm{A}^{\prime} B}$, a variável observada tem distribuição aproximada de " $t$ ", com $n$ ' graus de liberdade. Nesse caso, n'é obtido pela aproximação proposta por Satterthwaite em 1946

$$
n^{\prime}=\frac{[\operatorname{QMRes}(a)+(b-1) \operatorname{QMRes}(b)]}{\frac{[\operatorname{QMRes}(a)]^{2}}{n_{a}}+\frac{(b-1)^{2}[\operatorname{QMRes}(b)]^{2}}{n_{b}}}
$$

Para COCHRAN e COX (1976), que apresentam resultados análogos aos de PIMENTEL GOMES (1976), os quocientes entre as diferenças de tratamentos principais dentro de tratamentos secundários e os respectivos erros-padrão, não têm distribuição "t", podendo, no entanto, ser obtida uma aproximação razoável através de: 


$$
t=\frac{(-1) E_{b} t_{b}+E_{a} t_{a}}{(-1) E_{b}+E_{a}}
$$

onde $t_{a}$ e $t_{b}$ são, respectivamente, os nîveis de significância de $t$ correspondentes aos graus de liberdade do erro a $\left(E_{a}\right)$ e do erro b $\left(E_{b}\right)$, com B tratamentos secundārios.

Também TAYLOR (1959), com base em estudos desenvolvi dos por Whishart em 1940; Welch em 1947 e 1949; e Aspin em 1948; apresenta o mesmo resultado que Satterthwaite para o número de graus de liberdade da variável observada " $t$ ":

$$
\frac{1}{F}=\frac{C^{2}}{(J-1)(K-1)}+\frac{(1-C)^{2}}{(J-1) K(L-1)},
$$

onde: $\mathrm{F}=$ número de graus de liberdade;

$$
\begin{aligned}
& J=\text { nümero de blocos casualizados; } \\
& K=\text { nümero de tratamentos principais; } \\
& \mathrm{L}=\text { nümero de tratamentos secundários; }
\end{aligned}
$$

e

$$
C=\frac{\frac{2}{\mathrm{JL}} \operatorname{QMRes}(\mathrm{a})}{\frac{2}{\mathrm{JL}} \operatorname{QMRes}(\mathrm{a})+\frac{2(\mathrm{~L}-1)}{\mathrm{JL}} \operatorname{QMRes}(\mathrm{b})}
$$

\section{1 .4 - Anāitise de variāncia}

Segundo CHAKRABARTI (1962), HARTER (1961), LEAL(1979), dentre outros, sob a hipótese de normalidade dos erros, as somas de quadrados de interesse săo independentes e distribuidas como qui- 
-quadrados, nos ensaios com parcelas subdivididas em blocos casuali zados. Assim, para CHAKRABARTI (1962), num ensaio como aquele descrito em 2.1.3, encontra-se, através da Regra de Cochran, que a soma de quadrados devida ans tratamentos principais e a soma de quadrados devida ao resíduo (a) são independentes e distribuidas segun do qui-quadrados com $(\alpha-1)$ e $(\alpha-1)(r-1)$ graus de liberdade respecti vamente.

De modo análogo, as somas de quadrados relativas aos tratamentos secundārios e à interação tratamentos principais $\mathrm{x}$ tratamentos secundārios são independentes da soma de quadrados do resi duo (b) e cada qual é distribuida segundo um qui-quadrado com $(\beta-1)$, $(\alpha-1)(\beta-1)$ e $\alpha(r-1)(\beta-1)$ graus de liberdade, respectivamente.

Com base nessas propriedades, são obtidos os valores adequados para os testes "F", envolvendo tratamentos principais,tra tamentos secundārios e interação tratamentos principais $x$ tratamentos secundārios.

Para LEAL (1979), geralmente as hipöteses de interes se, em ensaios com parcelas subdivididas, são:

$$
\begin{array}{ll}
H_{0}(1): \quad t_{i}=0 & , i=1, \ldots, a \text { tratamentos principais; } \\
H_{0}(2): \quad t_{k}^{\prime}=0 & , k=1, \ldots, b \text { tratamentos secundārios; } \\
H_{0}(3): \delta_{i k}=0 & , i=1, \ldots, a ; k=1, \ldots, b . \\
\text { Os resíduos apropriados para testar essas hipóteses }
\end{array}
$$

são evidentes quando se observam as, variâncias descritas em 2.1.3. 
Se a hipötese de nulidade relativa aos efeitos de tra tamentos principais é rejeitada, conclui-se pela significância de ao menos um dentre os contrastes entre tratamentos. Nesse caso torna-se possîvel a utilização dos métodos de comparações múltiplas, como os de Tukey, Duncan, Scheffé, etc.

Sobre a eficiência dos ensaios com parcelas subdivididas em blocos casualizados, em relação aos ensaios em blocos casualizados, KEMPTHORNE (1950) mostra que, para tratamentos princi pais distribuidos em $\underline{\mathbf{r}}$ blocos e $\underline{\mathrm{s}}$ tratamentos secundārios, com a de composição seguinte:

\begin{tabular}{lcc}
\hline Fonte de Variação & G.L. & Q.M. \\
Blocos & $r-1$ & \\
Tratamentos principais (T) & $t-1$ & W \\
Erro (a) & $(r-1)(t-1)$ & \\
Tratamentos secundärios $(S)$ & $s-1$ \\
Tx S & $(t-1)(s-1)$ \\
Erro (b) & $(r-1) t(s-1)$ \\
\hline
\end{tabular}

a eficiência é calculada por

$$
E^{\prime}=\frac{(t-1) W+t(s-1) E}{t s-1} .
$$


Confirmando esse resultado, CHAKRABARTI (1962) apresenta, para o experimento descrito em 2.1.3,

$$
E=\frac{(\alpha-1) \sigma_{2}^{2}+\alpha(\beta-1) \sigma_{1}^{2}}{\alpha \beta-1} .
$$

Por sua vez, FEDERER (1955) propõe uma fórmula para calcular a eficiência dos ensaios em parcelas subdivididas com rela ção aos ensaios em blocos casualizados:

$$
E=\frac{(p-1) E_{a}+p(q-1) E_{b}}{(p q-1) E_{b}},
$$

para $p$ niveis do tratamento principal e $q$ niveis do tratamento secundärio, erro a $\left(E_{a}\right)$ e erro b $\left(E_{b}\right)$.

Naturalmente as três fórmulas acima são fundamentalmente iguais.

\section{2 - Ensaios em Blocos Incompletos Balanceados}

\subsection{1 - Generalidades}

Segundo TAYLOR (1957) e COCHRAN e COX (1976), dentre outros, os delineamentos em blocos incompletos foram introduzidos por Yates em 1936, com o objetivo de eliminar a heterogeneidade intrablocos, através da diminuição do número de parcelas.

Para PIMENTEL GOMES (1976), além da heterogeneidade 
a ser eliminada, existem ainda outros tipos de limitações que induzem à formação dos blocos incompletos, como o exemplo que foi citado em 1.1, quando se tomam leitegadas como blocos e existe o in teresse de se testar um grande nümero de tratamentos.

CHAKRABARTI (1962) define um delineamento em blocos incompletos balanceados (BIB), como um arranjo de $\underline{v}$ tratamentos dis tribuidos em $\underline{b} b l o c o s$ de $\underline{k}$ parcelas cada $(k<v)$, onde cada tratamento ocorre uma única vez em $\underline{r}$ dos $\underline{b}$ blocos $(r<b)$, e dois tratamentos quaisquer ocorrem juntos $\underline{\lambda}$ vezes, no experimento.

Ademais, segundo RAGHAVARAO (1971), dentre outros, devem ser observadas as condições de balanceamento demonstradas por Fisher em 1940:

$$
b k=r v \quad \text { e } \quad \lambda(v-1)=r(k-1) \text {. }
$$

Por sua vez, DEY (1975) define um ensaio em blocos incompletos como um BIB, se nele for possível estimar-se qualquer contraste entre tratamentos com a mesma precisão.

De modo geral, os autores são unânimes em apregoar a existência de três tipos básicos de ensaios em BIB, assim constitui dos:

BIB tipo I: os blocos podem ser arranjados em repetições;

BIB tipo II: os blocos não podem ser arranjados em repetições mas podem ser arranjados em grupos de repetições;

BIB tipo III: os blocos não podem ser arranjados em repetições nem em grupos de repetições. 
No entanto, alguns autores, como COCHRAN e COX (1976), acrescentam ainda dois tipos de ensaios em BIB:

BIB tipo IV: para casos em que o número de tratamentos é igual ao número de blocos;

BIB tipo $V$ : para pequenos experimentos.

Sobre os ensaios em BIB tipo IV, CHAKRABARTI (1962) acrescenta que existe a vantagem adicional da simplificação inserida nos cálculos, dada a simetria resultante da igualdade.

\subsection{2 - Matriz de variâncias e covariâncias}

Segundo PIMENTEL GOMES (1967), dentre outros, a matriz de dispersão para tratamentos é dada por:

$$
D(\widehat{T})=M^{-1} \mathrm{CM}^{-1} \sigma^{2}
$$

onde, $\mathrm{M}^{-}$é uma matriz diagonal com elementos $\mathrm{m}_{\mathrm{i}}=\frac{\mathrm{k}}{\lambda \mathrm{v}}$ e de dimensões $v \times v .(v=n$ ? de tratamentos $)$;

C é uma matriz singular, de característica igual a $(v-1)$, e com elementos

$$
c_{i i^{\prime}}=\left\{\begin{array}{l}
r(1-1 / k) \\
-\lambda / k \quad \text { se } i=i^{\prime}
\end{array}\right.
$$

Desse modo, $D(\hat{T})$ é constituída dos seguintes elementos:

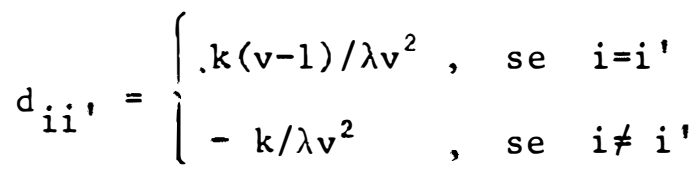


A variância da diferença entre os efeitos estimados de um par qualquer de tratamentos, é obtida de

$$
v\left(\hat{E}_{i}-\hat{E}_{i^{\prime}}\right)=\frac{2 k}{\lambda v} \sigma^{2},
$$

que é um resultado análogo àquele obtido por CHAKRABARTI (1962).

\subsection{3 - Variāncia da função linear estimāvel}

Sob esse aspecto, PIMENTEL GOMES (1967) mostra que, dada a função linear estimável dos parâmetros $P^{\prime} \hat{\tau}$, então $V\left(P^{\prime} \hat{\tau}\right)$ pode ser obtida diretamente da matriz $\mathrm{M}^{-1}$ :

$$
V\left(P^{\prime} \hat{\tau}\right)=P^{\prime} M^{-1} P \sigma^{2}
$$

Assim, por exemplo, se a função linear estimável é um contraste do tipo $\hat{t}_{1}-\hat{t}_{2}$, obtem-se

$$
\begin{aligned}
& V\left(P^{\prime} \hat{\tau}\right)=[1,-1,0, \ldots, 0]\left[\begin{array}{ccccc}
k / \lambda v & 0 & 0 & \ldots & 0 \\
0 & k / \lambda v & 0 & \ldots & 0 \\
\ldots \ldots & \ldots \ldots \ldots
\end{array}\right]\left[\begin{array}{c}
1 \\
0 \\
-1 \\
\ldots \\
0
\end{array}\right] \sigma^{2} \\
& \therefore V\left(P^{\prime} \bar{\tau}\right)=V\left(\hat{E}_{1}-\hat{E}_{2}\right)=\frac{2 k}{\lambda v} \sigma^{2},
\end{aligned}
$$

resultado idêntico ao obtido em 2.2.2 para estimadores de variância minima. 


\subsection{4 - Anāitise de Variāncia}

A revisão da bibliografia mostrou que os autores são unânimes no tocante à decomposição da soma de quadrados, na anālise de variância. Assim, tudo se passa como se a anälise fosse feita pa ra um ensaio em blocos casualizados (completos), com a diferença de que ocorre o ajuste da S.Q. de tratamentos e nos casos de BIB tipo I e II ocorre ainda a decomposição da S.Q. de blocos.

Para ensaios em BIB tipo III não hã decomposição,pois não existe o arranjo dos blocos em repetições ou em grupos de repetições.

Existe, no entanto, uma diferença concentual entre as linhas induzidas por Pimentel Gomes ou por Cochran e Cox, confor me descrito em 2.2.1, no sentido de que enquanto aquele ajusta a so ma de quadrados devida aos efeitos de tratamentos, estes ajustam a soma de quadrados devida aos efeitos de blocos.

Neste estudo, adotou-se o ajuste para a soma de quadrados de tratamentos que, segundo PIMENTEL GOMES (1976), é obtida por

$$
\operatorname{SQt}(a j \cdot)=\hat{\tau}^{\prime} Q
$$


19.

onde,

$$
\hat{i}=M^{-1} Q \quad \text { e } Q=T-N A^{-1} B \text {, }
$$

sendo, $\mathrm{T}$ e $\mathrm{B}$ os vetores dos totais observados para tratamentos e blo cos, respectivamente;

A, a matriz dos "tamanhos" dos blocos;

$\mathrm{N}$, a matriz de incidência dos tratamentos nos blocos.

No tocante à eficiência dos ensaios em blocos incompletos balanceados em relação aos ensaios em blocos casualizados, CHAKRABARTI (19h2), COCHRAN e COX (1976), PIMENTEL GOMES (1976), dentre outros, sajo concordantes ao apontar

$$
E=\frac{\lambda v}{r k}<1
$$

\section{3 - Ensaios com Parcelas Subdivididas em Blocos Incompletos}

\subsection{1 - Generalidades}

Assim como citado em 1.2, notou-se uma certa lacuna no tocante às obras publicadas sobre os ensaios em parcelas subdivi didas em blocos incompletos.

Para GILL (1978), um dos primeiros estudos realizados nessa área é devido a Robinson, e foi desenvolvido em 1967.

Em seu estudo, ROBINSON (1967) propôs um ensaio envolvendo $t$ níveis do tratamento prịcipal A e $\underline{s}$ niveis do tratamento secundário B. Entào, para $\ell=1, \ldots, s$, o autor distribui os $s$ 
níveis do tratamento secundārio em $\underline{k}$ subparcelas $(k<s)$ de cada parcela, segundo um esquema de blocos incompletos balanceados, considerando cada parcela como um bloco incompleto para tratamentos se cundārios. Sob essas condições, mostra que

$$
\lambda=R(k-1) /(s-1),
$$

onde $R=\frac{r k}{s}$ e $\underline{r}$ representa o nümero de parcelas que receberam 0 tratamento A. Nesse estudo levanta a hipótese de se estudar o caso em que os tratamentos principais estão dispostos em blocos incomple tos, mas não apresenta a discussão do problema.

Por sua vez, REES (1969), utilizando ensaios baseados no produto de Kronecker, enfoca o problema do confundimento nos ensaios em parcelas subdivididas e citando como precursores do processo Finey (1946) e Kempthorne (1947), alega que, sob certas condi ções, a análise de ensaios dessa natureza muito se assemelha à análise de ensaios com parcelas subdivididas em blocos incompletos.

Para GILL (1978), em determinadas āreas de pesquisa, como por exemplo a ārea de Fisiologia Reprodutiva, tem havido um in teresse crescente em torno dos experimentos com parcelas subdivididas em blocos incompletos. O autor argumenta, citando exemplos nos quais animais são designados aleatoriamente aos niveis de um tratamento principal, A, ou à combinação de dois ou mais tratamentos. Sob essas condições, alega que cada animal poderia constituir um bloco incompleto, pois, como é desejável, nesse tipo de experimentos, um unico animal não recebe todos os tratamentos. 


\section{3 .2 - 0 erro experimental}

Segundo GILL (1978), para aqueles ensaios por ele des critos e citados em 2.3.1, a estrutura devida às causas básicas de variação têm sido muitas vezes indevidamente simplificadas. Assim, para o autor, essa injustificada simplificação tem se processado ge ralmente de dois modos, nos ensaios em Fisiologia Reprodutiva:

a) Anālise dos dados em fatorial como se o experimento fosse inteiramente casualizado, em lugar de uma anālise considerando parcelas subdivididas.

b) Ignorância da correlação entre os erros, induzida pela repe tição das observações.

Quando a estrutura de "split-plot" não é reconhecida, afirma o autor, o experimentador executa a análise como se cada observação houvesse sido obtida com base em diferentes indivíduos. Des se modo, falha na separação do erro aleatório existente dentro da variação entre diferentes indivíduos e também na variação dentro de cada indivíduo. Consequentemente, uma aparente significância observada na diferença entre duas médias dos tratamentos, aos quais os animais foram designados, muitas vezes pode ser grosseiramente exage rada. Sob esse aspecto, o autor exemplifica citando a diminuição de sensibilidade que ocorre nos testes que envolvem interações entre tratamentos principais e secundários.

Assim, considerando o caso em que os animais são to- 
mados como blocos incompletos, o autor propõe o modelo:

$$
y_{i j k}=\mu+\alpha_{i}+D_{(i) j}+\beta_{k}+(\alpha \beta)_{i k}+(D B)_{(i) j k}+E_{i j k}
$$

para $i=1,2, \ldots, a ; j=1,2, \ldots, r($ por $i) ; k=1,2, \ldots, b$ e $n=a r b$;

onde $\alpha_{i} \bar{e}$ o efeito do i-ésimo nível do tratamento $A$ ao qual os animais são designados ao acaso;

$D_{(i) j} \bar{e}$ o efeito aleatório dos indivíduos dentro de A, que corres ponde ao erro (a);

$\beta_{k}$ è o efeito do k-ésimo nível do tratamento $B$, nos animais; $(\alpha \beta)_{i k}$ é o efeito da interação entre o i-ésimo nível do tratamento A e o k-ésimo nível do tratamento $B$, sobre os animais;

(DB) (i) jk é o efeito da interação entre animais e níveis do tratamento $B$, que segundo autor deve ser isolado do erro (b);

$E_{i j k}$ é o erro casual atribuído à observação $y_{i j k}$, ou erro (b).

Então, concordando com CHAKRABARTI (1962) e COCHRAN

e $\operatorname{cox}$ (1976), que desenvolveram seus estudos para ensaios em "Split-plot'com blocos completos, GILL (1978) defende a hipótese de que o erro experimental para parcelas, é do tipo

$$
[1+(b-1) \rho] \sigma^{2}
$$

e para subparcelas, tem a forma

$$
(1-\rho) \sigma^{2}
$$




\subsection{3 - Funções 1 ineares estimāveis e suas variäncias}

Para ROBINSON (1967), as diferenças entre médias estimadas para efeitos de tratamentos, envolvern quantidades do tipo

$$
\bar{z}_{i \ell}=\frac{Y_{i} .}{r k}+\frac{W Q_{i l}+\frac{W^{\prime}}{k}\left(T_{i \ell}-\frac{R}{r} Y_{i . .}\right)}{R E W+\frac{R-\lambda}{k} W^{\prime}}
$$

$$
\begin{aligned}
\operatorname{com} Q_{i \ell} & =y_{i, l}-\frac{1}{k} \sum_{j} n_{i j}^{\ell} y_{i j} . \\
T_{i \ell} & =\sum_{j} n_{i j}^{\ell} y_{i j} . \\
R E & =R-\frac{R}{k}+\frac{\lambda}{k}
\end{aligned}
$$

obtidas do desenvolvimento do modelo

$$
y_{i j}=n_{i j}^{\ell}\left[\mu+\alpha_{i}+e_{i j}+\beta_{\ell}+(\alpha \beta)_{i \ell}+\delta_{i j \ell}\right],
$$

onde, para $i=1, \ldots, t ; j=1, \ldots, r ; l=1, \ldots, s$;

$y_{i j \ell}$ é o valor observado da subparcela que recebeu o $\ell$-ésimo nivel do tratamento secundário $B$, na j-ésima repetição do i-ésimo nivel do tratamento principal A;

$\mu$ é o efeito médio;

$\alpha_{i}$ é o efeito do i-ésimo nível de A;

$\beta_{\ell}$ é o efeito do l-ésimo nível de $B$;

$(\alpha \beta)_{i j}$ é o efeito da interação do i-ésimo nỉvel de A com o l-ési mo nivel de $B$;

$e_{i j}$ e $\delta_{i j}$ são variáveis aleatóriàs não correlacionadas, ambas com média zero e com variâncias $\sigma_{e}^{2}$ e $\sigma_{\delta}^{2}$, respectivamente; 


$$
n_{i j}^{\ell}= \begin{cases}1, & \text { se o l-ésimo nivel de } B \text { ocorre na } j \text {-ésima repetição } \\ & \text { do i-ésimo nivel de } A,\end{cases}
$$

Sob esse prisma, ROBINSON (1967) mostra que a variân cia para a diferença entre tratamentos è dada por

$$
V\left(\bar{z}_{i \ell}-\bar{z}_{i \ell^{\prime}}\right)= \begin{cases}\frac{2}{R E W+\frac{R-\lambda}{k} W^{\prime}} & \text {, se } i=1^{\prime} \\ \frac{2}{r k W^{\prime}}+\frac{2(s-1)}{s\left(R E W+\frac{R-\lambda}{k} W^{\prime}\right)} & \text {, se } i \neq i^{\prime}\end{cases}
$$

onde, $W=\frac{1}{\sigma_{\delta}^{2}}$ e $W^{\prime}=\frac{1}{\sigma_{\delta}^{2}+k \sigma_{e}^{2}}$,

e R, r, k, s e $\lambda$ são definidas como em 2.3.1.

Mostra ainda, que para as diferenças marginais entre médias, as variâncias são obtidas de

$$
V\left(\bar{z}_{i},-\bar{z}_{i},\right)=\frac{2}{r k W^{\prime}}
$$

e

$$
V\left(\bar{z} \cdot \ell-\bar{z} \cdot \ell^{\prime}\right)=\frac{2}{t R E W+t \frac{R-\lambda}{k} W^{\prime}}
$$

Para GILL (1978), as estimativas dos erros-padrão pä ra as médias dos tratamentos $A, B$ e interação $A B$, são dadas respectivamente por

$$
s\left(\bar{y}_{i \ldots}\right)=\left[\left(Q_{D / A}\right) /(b r)\right]^{1 / 2}
$$




$$
\begin{aligned}
& s\left(\bar{y}_{\ldots k}\right)=\left[\left(Q M_{E}\right) /(a r)\right]^{2 / 2} \\
& s\left(\bar{y}_{i \cdot k}\right)=\left[\left(Q M_{E}\right) / r\right]^{1 / 2}
\end{aligned}
$$

$$
\text { onde, } \begin{aligned}
Q_{D / A} & =\bar{s}_{k}^{2}+(b-1) \bar{s}_{k k^{\prime}} ; \\
Q_{E} & =\bar{s}_{k}^{2}-\bar{s}_{k k^{\prime}}
\end{aligned}
$$

e

$$
\begin{aligned}
\bar{s}_{k}^{2}= & \text { variância média amostral entre individuos para um da- } \\
& \text { do grupo de tratamentos principais (A); } \\
\bar{s}_{k^{\prime}{ }^{\prime}}= & \text { covariância média amostral dentro do grupo de trata- } \\
& \text { mentos } B,\left(k \neq k^{\prime}\right) .
\end{aligned}
$$

Desse modo, o erro padrão adequado para testar-se a diferença entre duas médias de tratamentos principais é:

$$
\left[\left(2 \mathrm{QM}_{E}\right) / \mathrm{r}\right]^{1 / 2}
$$

E para tratamentos secundários

$$
\left[\left(2 \mathrm{QM}_{\mathrm{D} / \mathrm{A}}\right) / \mathrm{r}\right]^{1 / 2} \text {. }
$$

\subsection{4 - Anālise de variāncia}

ROBINSON (1967) fornece, para o experimento citado em 2.3.1, o seguinte quadro para anālise de variância, considerando as esperanças dos quadrados médios: 


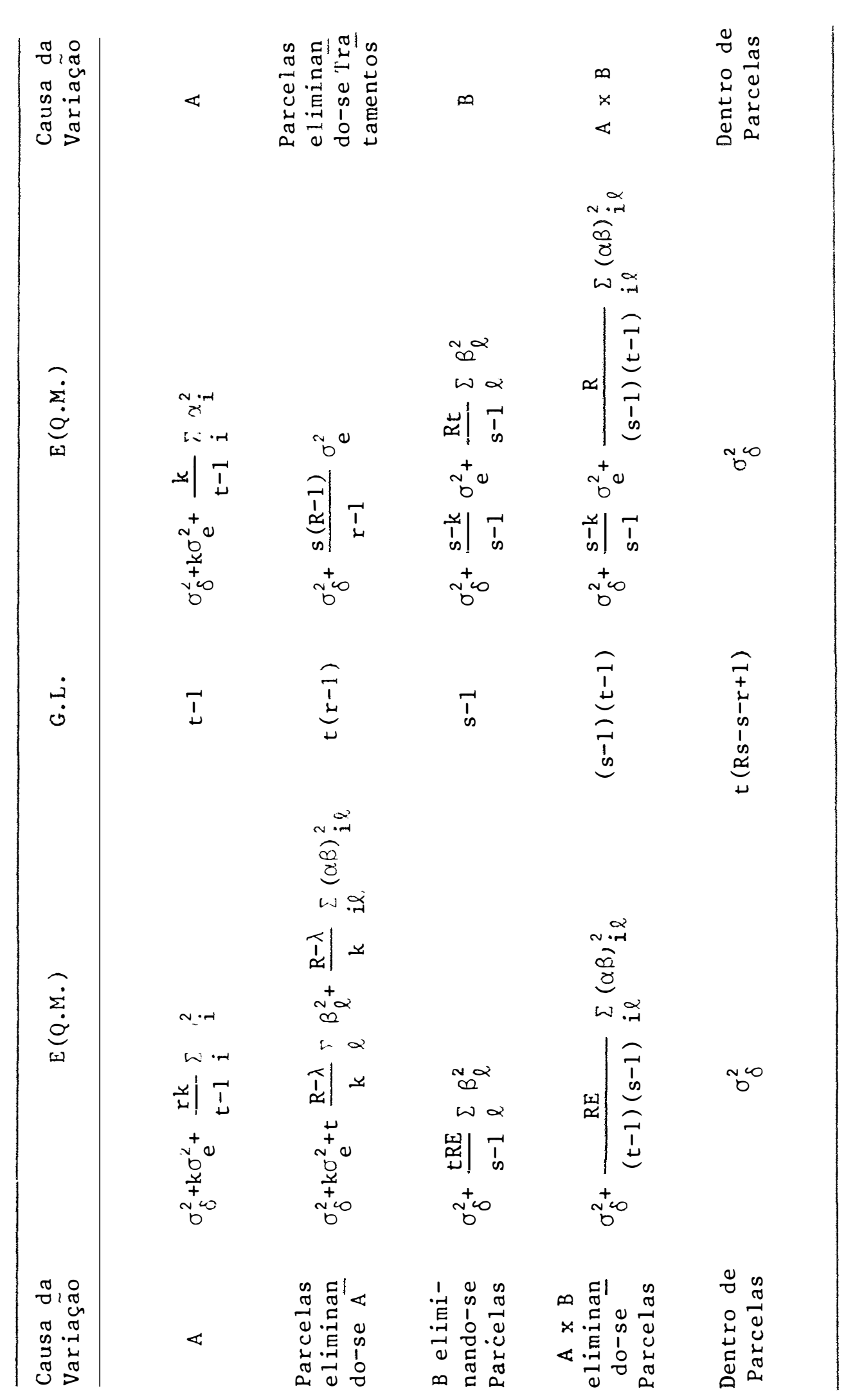

26. 
Desse modo, para o autor, existem os testes exatos para $\beta_{\ell}=0$, $\forall \ell$ e $(\alpha \beta)_{i \ell}=0, \forall i, \ell$, com base no erro existente "dentro de Parcelas".

No entanto, para a hipótese $\alpha_{i}=0, \forall i$, não existe um teste exato, a menos que o experimento seja repetido. Então, a soma de quadrados para "Parcelas eliminando-se A" pode ser partidaem dois componentes, que podem ser calculados para a soma de quadrados da di ferença entre totais de parcelas correspondentes em duas ou mais repetições do experimento.

Por sua vez, GILL (1978) apresenta o seguinte quadro de anālise refletindo a correlação constante entre quaisquer duas subparcelas de uma mesma parcela.

\begin{tabular}{lcc}
\hline Causa da Variação & G.L. & E(Q.M.) \\
\hline Entre Indivíduos & ar-1 & \\
Tratamentos (A) & $a-1$ & {$[1+(b-1) p] \sigma^{2}+\left(\sum_{i=1}^{a} \alpha_{i}^{2}\right) / \gamma_{A}$} \\
Erro (a) & $a(r-1)$ & {$[1+(b-1) p] \sigma^{2}$} \\
\hline
\end{tabular}

Dentro de Indivíduos ar $(b-1)$

Tratamentos (B) $\quad b-1 \quad(1-\rho) \sigma^{2}+\sigma_{D B}^{2}+\left(\operatorname{ar} \sum_{k=1}^{b} B_{k}^{2}\right) / \gamma_{B}$
$A \times B$
$(a-1)(b-1)$
$(1-\rho) \sigma^{2}+\sigma_{D B}^{2}+\left[r \sum_{i=1}^{a} \sum_{k=1}^{b}(\alpha B)_{i k}^{2}\right] / \gamma_{A B}$
Erro (b)

$$
a(r-1)(b-1) \quad(1-\rho) \sigma^{2}+\sigma_{D B}^{2}
$$


Assim como COCHRAN e COX (1976), o autor ressalta que essa correlação é geralmente positiva e, sob essa condição, o erro (b) é considerado menor que o erro (a).

Desse modo, inferências sobre $B$ e $A B$ podem ser feitas com maior precisào, do que sobre efeitos médios do tratamento $A$. No tocante à hipótese de nulidade para a interação $\mathrm{H}_{\mathrm{o}}:(\alpha B)_{i k}=0$, GILL (1978) aconselha o teste estatístico:

$$
F=\frac{Q M A B}{Q M_{E}},
$$

comparāvel a $\mathrm{F}_{x \%} ;(\mathrm{a}-1)(\mathrm{b}-1) ;[\mathrm{a}(\mathrm{r}-1)(\mathrm{b}-1)]$

Para testar $H_{0}: \alpha_{i}=0, \forall i$, o autor recomenda

$$
F=\frac{{ }^{M} A}{Q M_{D} / A},
$$

com valor crítico dado por $F_{\alpha \% ;(a-1) ;[a(r-1)]}$

$$
\begin{gathered}
E \text {, para } H_{0}: B_{k}=0 \text {, } \forall k \\
F=\frac{Q M_{B}}{Q M_{E}}
\end{gathered}
$$

com valor crítico $\mathrm{F}_{\alpha \% ;}(\mathrm{b}-1) ;[\mathrm{a}(\mathrm{r}-1)(\mathrm{b}-1)]$

Se a matriz de variāncias e covariâncias é uniforme para todos os níveis de $A$, mas não é homogênea para os níveis de $B$, a validade do teste $H_{0}: \alpha_{i}=0, \forall i$, näo é afetada, mas o teste esta tistico para $H_{0}: \beta_{k}=0$, Fil, deve ser comparado com o valor critico 


$$
F_{\alpha \% ; 1 ;[a(r-1)]}
$$

para um teste conservativo ou, então, seguir os procedimentos usuais da análise multivariada. 
3. MATERIAL

Apesar de inicialmente haver-se considerado este estudo sob o aspecto teórico, sentiu-se que a ilustraçäo dos procedimentos aqui apresentados seria desejāvel, no sentido de complementa sào do trabalho.

No entanto, dada a escassez de publicações sobre o assunto, conforme citado em (1.2), três opções foram consideradas, para a obtençào dos dados:

i) Montar-se um experimento em parcelas subdivididas com trata mentos principais dispostos em blocos incompletos balanceados.

ii) Simular-se um conjunto de dados de acordo com o modelo mate mático que rege esse tipo de experimento, proposto em (4.2).

iii) Proceder-se à adequação, através de reagrupamentos, de dados publicados que envolvessem ensaio com parcelas subdivididas em 
blocos (completos) casualizados.

Obviamente, a primeira opçäo mostrou-se mais adequam da que as demais, mas foi descartada, pois envolveria, além do tempo necessário para obtenção dos dados de campo, o conhecimento de conceitos na área específica em que se desenvolveria a pesquisa.

Descartou-se, também, a segunda opção, considerando-se que seria mais desejāvel a utilização de dados experimentais,mor mente no tocante à homogeneidade e uniformidade das matrizes de covariāncias. Sob esse aspecto considerou-se que para dados simulados, com base no modelo matemático, as matrizes de covariâncias, sem dúvida, teriam a estrutura prevista, pairando, no entanto, a expectativa sobre seu comportamento com dados experimentais sobre o mode 10 proposto.

Assim, mostrou-se mais oportuna a obtenção dos dados a partir do reagrupamento de dados já existentes em algum experimen to com parcelas subdivididas em blocos (completos) casualizados. Na turalmente essa terceira opção foi possível apenas porque o objetivo buscado no manuseio dos dados foi estritamente o de ilustrar a aplicação da metodologia abordada neste estudo.

Tomou-se, então, o conjunto de dados referentes à du ração do ciclo de ovulação de ratas, em horas, segundo a dieta e a idade, adaptados de GILL (1978), e que constam da Tabela 1.

Na disposição dos tratamentos principais (dietas), a- 
dotou-se o plano nümero 11.6 para blocos incompletos balanceados do tipo II, publicado por COCHRAN e COX (1976) e que pressupõe:

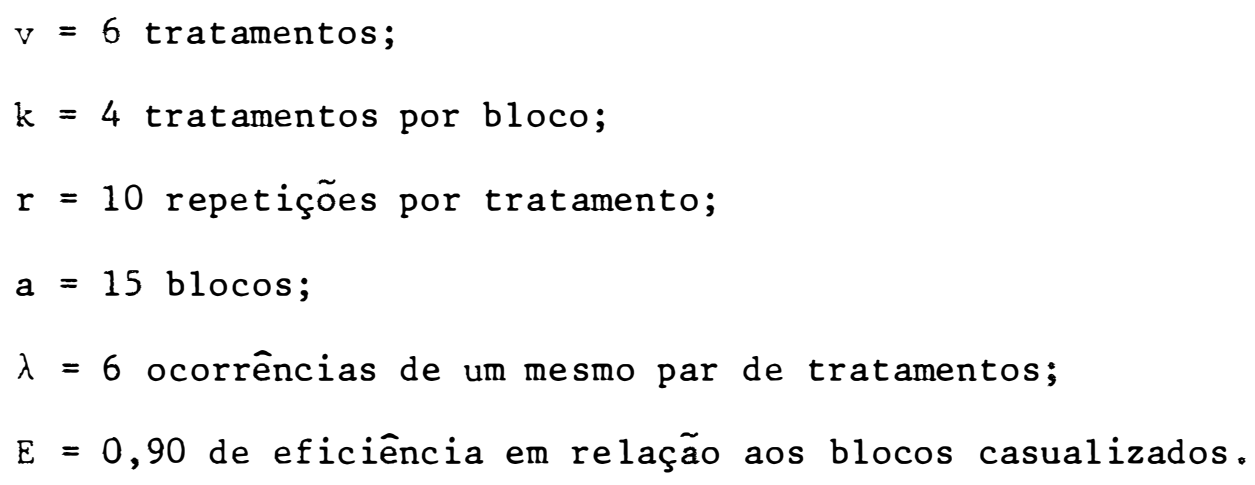



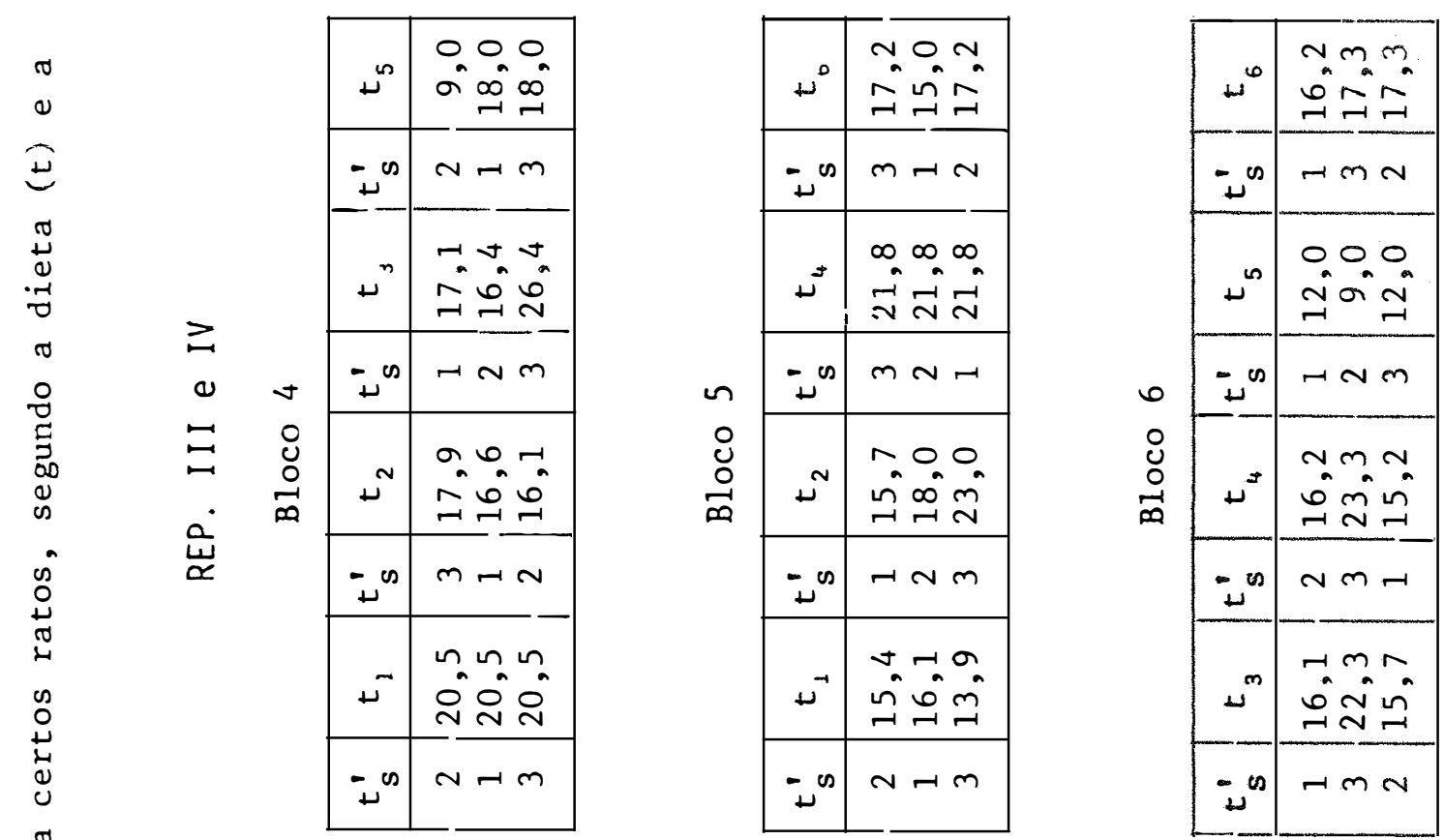

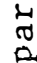

\%
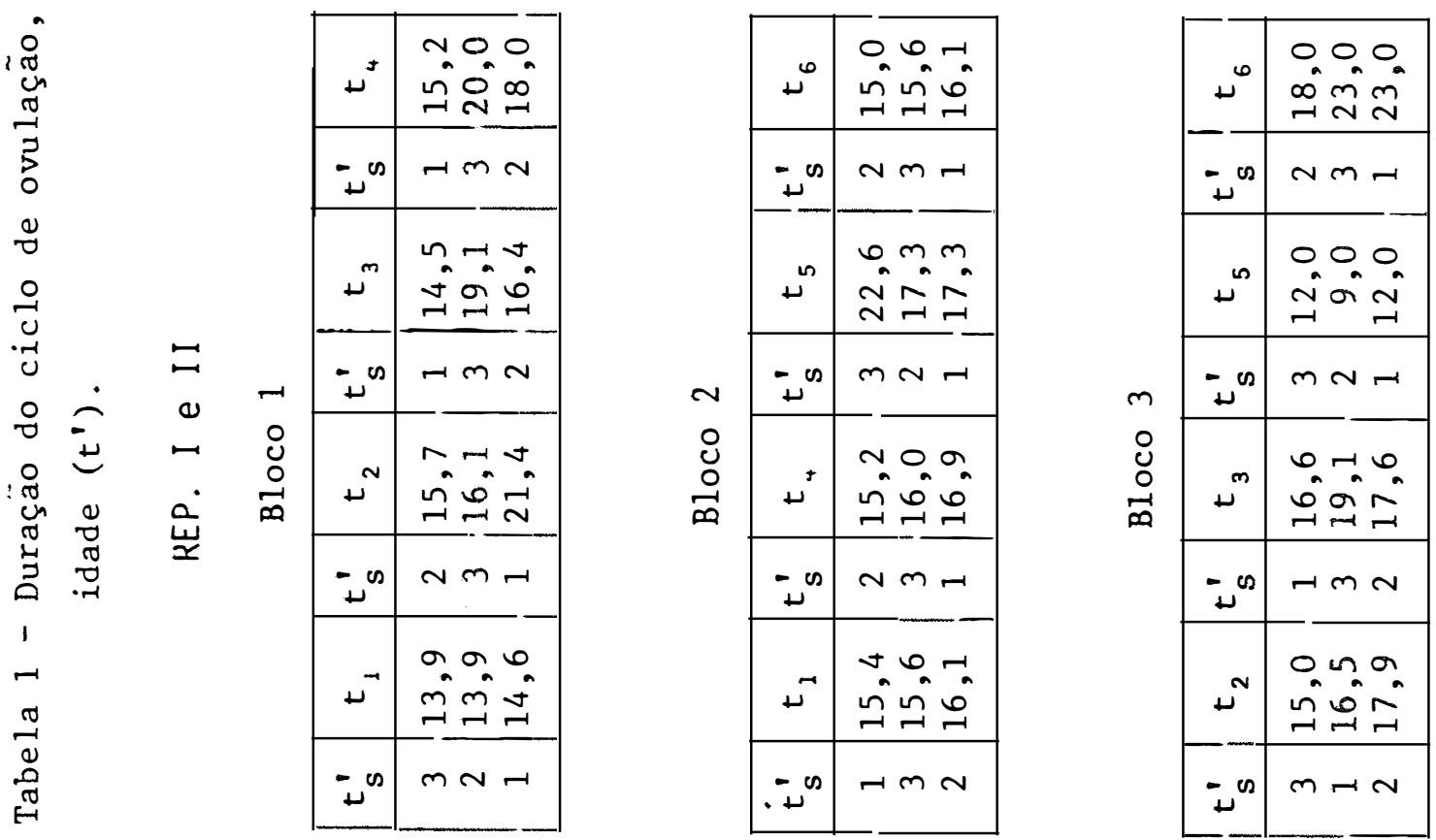
34.
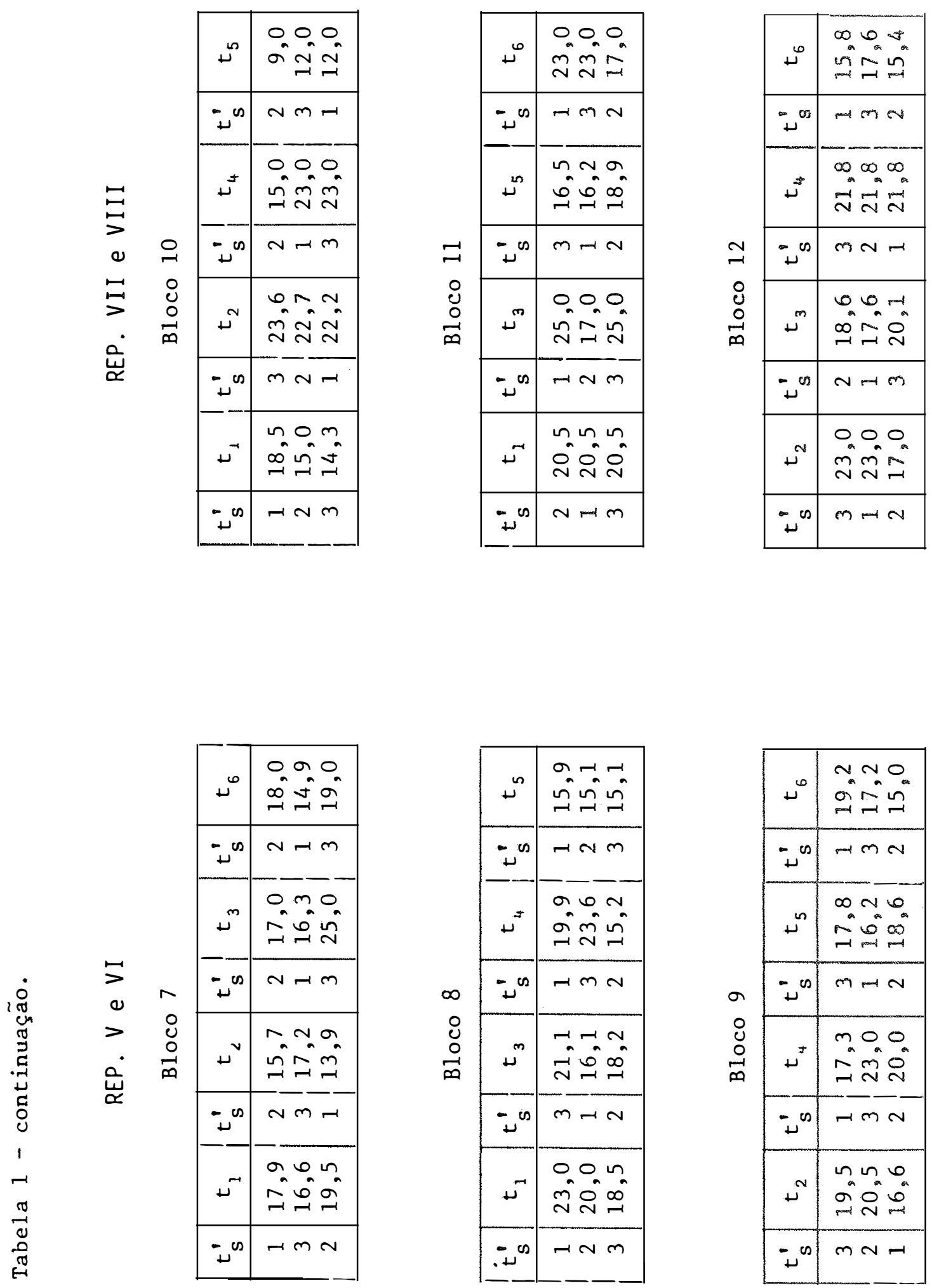

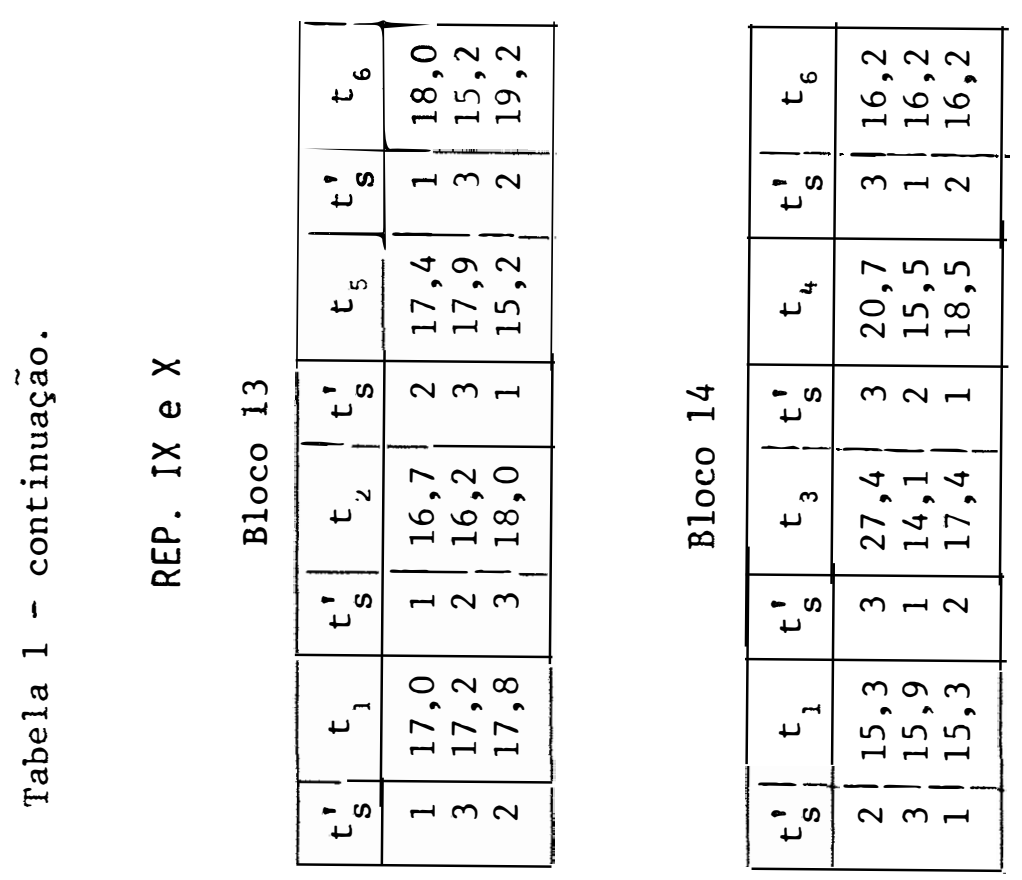

\begin{tabular}{|c|c|}
\hline$\omega^{n}$ & 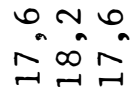 \\
\hline$=\infty$ & $m \sim r$ \\
\hline$L^{\top}$ & $\begin{array}{l}N a \sigma \\
\infty \\
\infty\end{array}$ \\
\hline$=\infty$ & $-m N$ \\
\hline$\nu^{m}$ & $\begin{array}{l}ت n \\
\tilde{n} \dot{\sim}\end{array}$ \\
\hline$-\infty$ & $\neg N m$ \\
\hline$H^{N}$ & 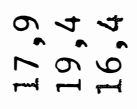 \\
\hline in & $N m-$ \\
\hline
\end{tabular}


4. METODOS

\section{1 - Introdução}

Para o desenvolvimento da metodologia, supôs-se um ensaio em Parcelas Subdivididas, no qual os $\underline{v}$ tratamentos principais estivessem dispostos em a blocos incompletos balanceados. Considerou-se, também, que o experimento envolvesse:

r repetições para cada tratamento principal;

k tratamentos principais por bloco;

u tratamentos secundários.

Ademais, supôs-se que o número de vezes em que cada par de tratamentos ocorresse num mesmo bloco fosse igual a $\lambda$, no ex perimento.

Tomando-se por base que num ensaio em blocos incom- 
completos as generalizações tornam-se geralmente difíceis e pouco inteligiveis, houve-se por bem construir um exemplo inicial e usá- lo como suporte para a construção das matrizes mais elaboradas, a par das generalizações. Com esse objetivo, tomou-se, sem perda de generalidade, o delineamento em blocos incompletos do tipo I,que em COCHRAN e COX (1976) recebe o número 11.1 , no qual estariam dispostos os tratamentos principais.

Assim, para

$v=4$ tratamentos principais no ensaio;

$k=2$ tratamentos principais por bloco;

$r=3$ repetições para cada tratamento principal;

$a=6$ blocos;

$\lambda=1$ ocorrência de par de tratamentos principais por blo co;

$u=5$ tratamentos secundärios no ensaio,

obteve-se o seguinte esquema: 

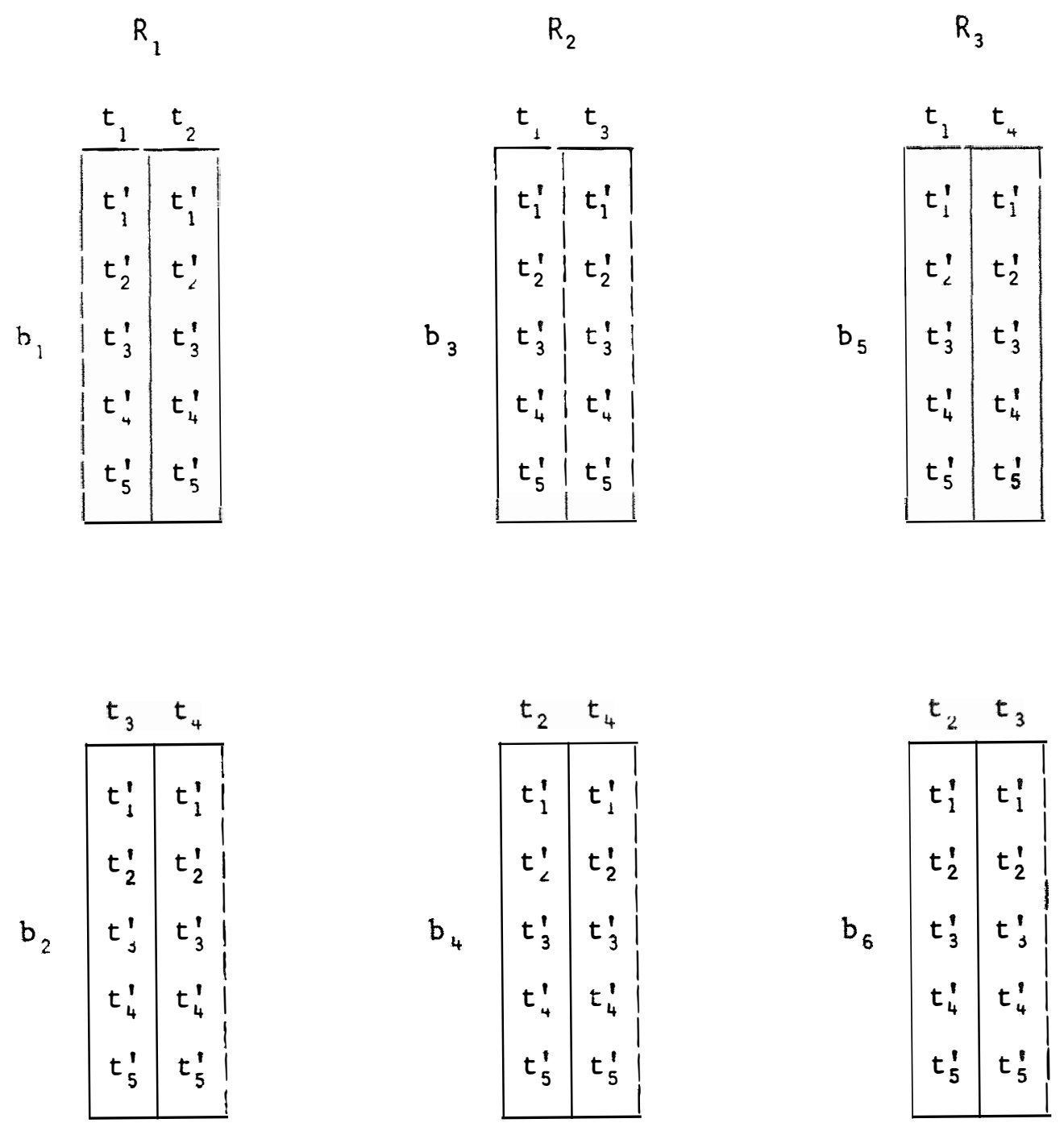

onde, $R_{1}, R_{2}$ e $R_{3}$ são as $r=3$ repetições nas quais estão agrupados os blocos;

$t_{i}, i=1, \ldots, 4$ representa o $i$-ésimo tratamento principal;

$t_{s}^{\prime}, s=1, \ldots, 5$ representa o $s$-ésimo tratamento secundärio;

$b_{j}, j=1, \ldots, 6$ representa o j-ésimo bloco. 
Naturalmente a fixação dos tratamentos secundārios às subparcelas teve apenas o objetivo didático de facilitar a interpre tação do diagrama. Na verdade, como abordado em 2.1 eles devem ser casualizados às subparcelas.

\section{2 - Modelo Matemático}

Tomou-se como modelo matemático, para esses ensaios, o modelo linear:

$$
y_{i j s}=m+t_{i}+b_{j}+e_{k(i j)}+t_{s}^{\prime}+\left(t t^{\prime}\right)_{i s}+e_{i j s}
$$

onde, para $i=1,2, \ldots, v ; j=1,2, \ldots, a ; s=1,2, \ldots, u$,

$y_{i j s}$ é o valor observado na subparcela que recebeu o s-ésimo tratamento secundário, dentro do i-ésimo tratamento prin cipal, no j-ésimo bloco;

m é o efeito da média geral;

$t_{i}$ é o efeito do i-ésimo tratamento principal;

$b_{j}$ é o efeito do j-ésimo bloco;

$e_{k(i j)}$ è o erro atribuido à k-ésima parcela do bloco $j$, que re cebeu o i-ésimo tratamento principal; caracterizado como componente do erro (a):

$t_{s}^{\prime}$ é o efeito do s-ésimo tratamento secundário;

$\left(t t^{\prime}\right)$ is é o efeito da interação entre o i-ésimo tratamento prin cipal e o s-ésimo tratamento secundário;

$e_{i j s}$ è o erro atribuido à observação $y_{i j s}$, caracterizado como componente do erro (b). 
Neste estudo, supôs-se como em CHAKRABARTI (1962), COCHRAN e COX (1976) e PIMENTEL GOMES (1976), entre outros, que:

$$
\sum_{i=1}^{v} t_{i}=\sum_{j=1}^{a} b_{j}=\sum_{s=1}^{u} t_{s}^{\prime}=\sum_{i=1}^{v}\left(t t^{\prime}\right)_{i s}=\sum_{s=1}^{u}\left(t t^{\prime}\right)_{i s}=0 .
$$

Considerou-se, também, como em CHAKRABARTI (1962), GILL (1978), LEAL (1979) e DINIZ (1980), a existência de uma corre-

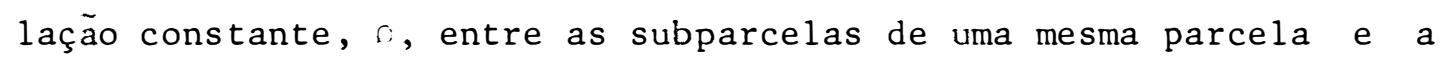
independência entre subparcelas de parcelas distintas.

Entào,

$$
\operatorname{Cov}\left(y_{i j s^{\prime}} y_{i} j^{\prime} s^{\prime}\right)=\left\{\begin{array}{l}
\sigma^{2}, \text { se } i=i^{\prime}, j=j^{\prime}, s=s^{\prime} \\
\rho \sigma^{2}, \text { se } i=i^{\prime}, j=j^{\prime}, s \neq s^{\prime} \\
0, \text { em outros casos }
\end{array}\right.
$$

Tomou-se esse modelo linear como base para qualquer um dos três casos considerados neste estudo, no tocante à distribuição dos tratamentos principais: BIB tipo I, II ou III. Desse modo, para os três casos, apenas as anālises de variâncias foram consideradas individualmente, pois ali se processaram as decomposições da SQ Blocos descritas em 2.2. 
Tomando-se o modelo linear $Y=X \theta+\varepsilon$, obteve-se $o$ sistema de equações normais

$$
X^{\prime} X \hat{\theta}=X^{\prime} Y
$$

onde, $X$ é a matriz dos coeficientes dos parâmetros, de dimensões (urv) $\times(v+a+u+u v+1)$;

Ê é o vetor das soluções de mínimos quadrados, para os efeitos dos parâmetros, de dimensões $(v+a+u+u v+1) \times(I)$;

Y é o vetor dos valores observados, de dimensões (urv) x (1).

Desse modo, efetuando-se a partição da matriz $x$, con forme PIMENTEL GOMES (1967), resultou

$$
x=\left[x_{1}, x_{2}, x_{3}, x_{4}, x_{5}\right] \text {, }
$$

onde, $x$, é o vetor que envolve a média geral, de dimensões (urv) $x$ x (1);

$x$ é a matriz dos coeficientes associados aos tratamentos prin cipais, de dimensões (urv) x (v);

$X_{3}$ é a matriz dos coeficientes associados aos blocos, de dimensöes (urv) x (a);

$X_{4}$ é a matriz dos coeficientes associados aos tratamentos secundārios, de dimensões (urv) x (u);

$x_{5}$ é a matriz dos coeficientes associados às interações ( $\left.t t^{\prime}\right)_{\text {is }}$, de dimensões (urv) x (uv). 
Assim, $X^{\prime} X$ resultou do tipo

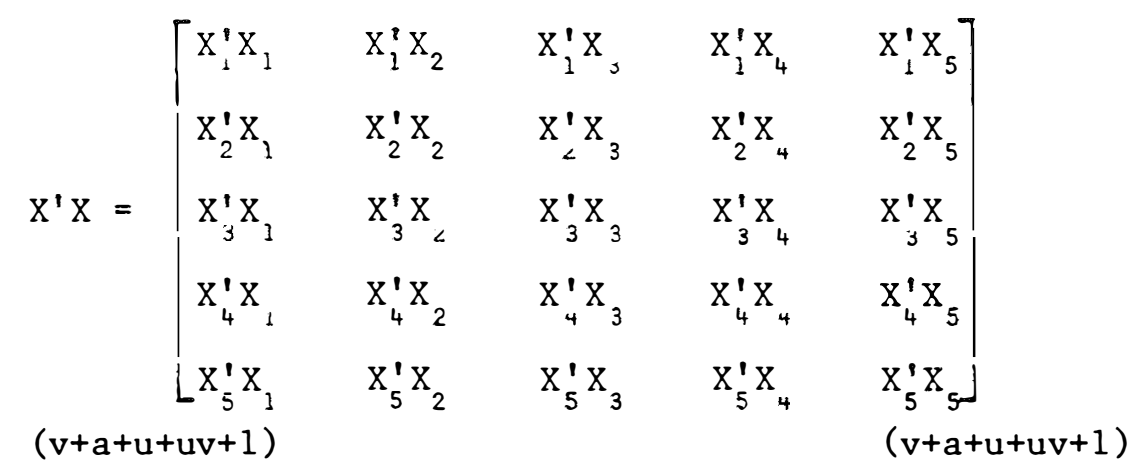

e suas submatrizes foram designadas por:

$$
\begin{aligned}
& \mathrm{X}_{1}^{\prime} \mathrm{X}_{1}^{\prime}=\text { urv }=\mathrm{n}=\text { nümero de subparcelas; } \\
& \left.\mathrm{X}_{1}^{\prime} \mathrm{X}_{2}=\mathrm{x}=\underset{(1)}{[\mathrm{ur}} \text {, ur, } \ldots, \underset{(\mathrm{v})}{\mathrm{ur}}\right] \text { vetor associado ao número de } \\
& \text { repetições dos tratamentos principais; } \\
& \left.\mathrm{X}_{1}^{\prime} \mathrm{X}_{3}=\mathrm{y}=\underset{\mathrm{uk}}{\mathrm{u}}, \mathrm{uk}, \ldots, \mathrm{uk} \overline{]}\right]=\text { vetor associado ao número de } \\
& \text { subparcelas por bloco; } \\
& \mathrm{X}_{1}^{\prime} \mathrm{X}_{4}=z=[\mathrm{rv}, \mathrm{rv}, \ldots, \mathrm{rv}] \text { = vetor associado ao nümero de } \\
& \text { repetições dos tratamentos secunciārios; }
\end{aligned}
$$

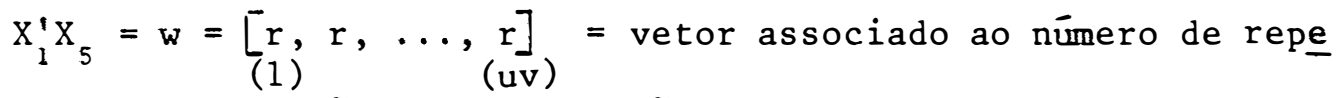

$$
\begin{aligned}
& \text { tições das interações; } \\
& X_{2}^{\prime} X_{2}=R=\left[\begin{array}{cccccc}
\text { ur } & 0 & 0 & \ldots & 0 & 0 \\
0 & \text { ur } & 0 & \ldots & 0 & 0 \\
\ldots & \ldots & \ldots & \ldots & \ldots & \ldots \\
0 & 0 & 0 & \ldots & 0 & \text { ur }
\end{array}\right]=u \cdot r \cdot I_{(v)} \text {, } \\
& \text { (v) }
\end{aligned}
$$

onde $I_{(v)}$ é uma matriz identidade de dimensão $\underline{v}$ (matriz das repetições dos tratamentos principais) 


$$
\begin{aligned}
& X_{2}^{\prime} X_{3}=N \text {, de dimensões }(v) x(a) \text {, onde } \\
& \qquad n_{i j}=\left\{\begin{array}{l}
u, \text { se o tratamento } i \text { ocorre no bloco } j, \\
0, \text { caso conträrio, }
\end{array}\right.
\end{aligned}
$$

é a matriz de incidência do i-ésimo tratamento principal no j-ésimo bloco.

No exemplo citado em (4.1), obteve-se:

$$
N=\left[\begin{array}{llllll}
5 & 0 & 5 & 0 & 5 & 0 \\
5 & 0 & 0 & 5 & 0 & 5 \\
0 & 5 & 5 & 0 & 0 & 5 \\
0 & 5 & 0 & 5 & 5 & 0
\end{array}\right]
$$

$$
\begin{aligned}
& X_{2}^{\prime} X_{u_{4}}=P=\left[\begin{array}{cccc}
r & r & \cdots & r \\
r & r & \ldots & r \\
\cdots & \ldots & \ldots & \ldots \\
r & r & \ldots & r
\end{array}\right]=r \underset{(v)}{E(u)} \\
& \text { (v) (u) }
\end{aligned}
$$

onde E è uma matriz cujos elementos são todos iguais à unida (v) (u)

de e P é a matriz do número de repetições de cada par ( $\left.t t^{\prime}\right)_{\text {is }}$.

$$
\mathrm{X}_{2}^{\prime} \mathrm{x}_{\mathrm{s}}=\mathrm{s}=\left[\begin{array}{cccccccccc}
\mathrm{r} & \ldots & \mathrm{r} & 0 & \ldots & 0 & \ldots & 0 & \ldots & 0 \\
0 & \ldots & 0 & \mathrm{r} & \ldots & \mathrm{r} & \ldots & 0 & \ldots & 0 \\
\ldots & \ldots & \ldots & \ldots & \cdots & \ldots & \ldots & \ldots & \ldots & \ldots \\
0 & \ldots & 0 & 0 & \ldots & 0 & \ldots & \mathrm{r} & \ldots & \mathrm{r}
\end{array}\right]_{(\mathrm{v})}
$$


é a matriz de incidencia dos tratamentos principais nos pares $\left(t t^{\prime}\right)$ is ${ }^{\circ}$

No exemplo em questão, obteve-se

$$
S=\left[\begin{array}{llllllllllllllllllll}
3 & 3 & 3 & 3 & 3 & 0 & 0 & 0 & 0 & 0 & 0 & 0 & 0 & 0 & 0 & 0 & 0 & 0 & 0 & 0 \\
0 & 0 & 0 & 0 & 0 & 3 & 3 & 3 & 3 & 3 & 0 & 0 & 0 & 0 & 0 & 0 & 0 & 0 & 0 & 0 \\
0 & 0 & 0 & 0 & 0 & 0 & 0 & 0 & 0 & 0 & 3 & 3 & 3 & 3 & 3 & 0 & 0 & 0 & 0 & 0 \\
0 & 0 & 0 & 0 & 0 & 0 & 0 & 0 & 0 & 0 & 0 & 0 & 0 & 0 & 0 & 3 & 3 & 3 & 3 & 3
\end{array}\right]
$$

(4)

$$
X_{3}^{\prime} X_{3}=A=\left[\begin{array}{cccccc}
u k & 0 & 0 & \cdots & 0 & 0 \\
0 & u k & 0 & \cdots & 0 & 0 \\
\ldots & \ldots & \ldots & \ldots & \ldots & \ldots \\
0 & 0 & 0 & \ldots & 0 & u k
\end{array}\right]_{(a)}^{(a)}\left(a \cdot k \cdot I_{(a)}\right.
$$

é a matriz diagonal do número de subparcelas por bloco,

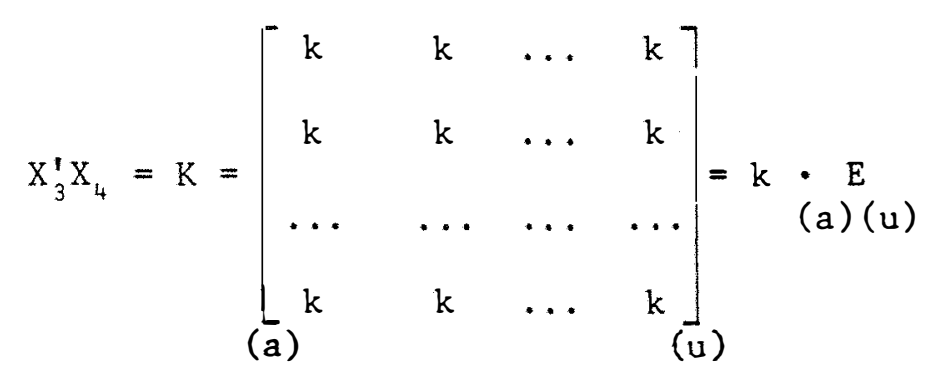

é a matriz controle do número de tratamentos principais por bloco,

$$
\begin{aligned}
& X_{3}^{\prime} X_{5}=v \text {, de dimensões (a) } x \text { (uv), onde } \\
& \qquad v_{j, i s}=\left\{\begin{array}{l}
1, \text { se }\left(t t^{\prime}\right)_{\text {is }} \text { ocorre no bloco } j, \\
\dot{0}, \text { caso conträrio, }
\end{array}\right.
\end{aligned}
$$


é a matriz de incidência da interação $\left(t t^{\prime}\right)$ is, no bloco $j$.

No exemplo inicial, obteve-se

$$
V=\left[\begin{array}{llllllllllllllllllll}
1 & 1 & 1 & 1 & 1 & 1 & 1 & 1 & 1 & 1 & 0 & 0 & 0 & 0 & 0 & 0 & 0 & 0 & 0 & 0 \\
0 & 0 & 0 & 0 & 0 & 0 & 0 & 0 & 0 & 0 & 1 & 1 & 1 & 1 & 1 & 1 & 1 & 1 & 1 & 1 \\
1 & 1 & 1 & 1 & 1 & 0 & 0 & 0 & 0 & 0 & 1 & 1 & 1 & 1 & 1 & 0 & 0 & 0 & 0 & 0 \\
0 & 0 & 0 & 0 & 0 & 1 & 1 & 1 & 1 & 1 & 0 & 0 & 0 & 0 & 0 & 1 & 1 & 1 & 1 & 1 \\
1 & 1 & 1 & 1 & 1 & 0 & 0 & 0 & 0 & 0 & 0 & 0 & 0 & 0 & 0 & 1 & 1 & 1 & 1 & 1 \\
0 & 0 & 0 & 0 & 0 & 1 & 1 & 1 & 1 & 1 & 1 & 1 & 1 & 1 & 1 & 0 & 0 & 0 & 0 & 0
\end{array}\right]
$$

$$
X_{4}{ }^{\prime} X_{4}=U=\left[\begin{array}{cccccc}
r v & 0 & 0 & \ldots & 0 & 0 \\
0 & r v & 0 & \ldots & 0 & 0 \\
\ldots & \ldots & \ldots & \ldots & \ldots & \ldots \\
0 & 0 & 0 & \ldots & 0 & r v
\end{array}\right]=r \cdot v \cdot I_{(u)}
$$

(u)

(u)

é a matriz diagonal associada ao número de repetições dos tratamentos secundärios.

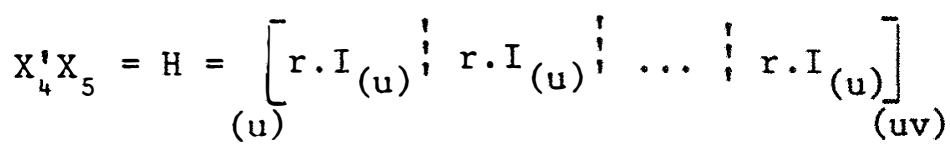

é a matriz de incidência dos tratamentos secundários nos pares $\left(t t^{\prime}\right)_{\text {is }}$

No exemplo em questão obteve-se: 
$\mathrm{H}=\left[\begin{array}{lllll:lllll:lllll:lllll}3 & 0 & 0 & 0 & 0 & 3 & 0 & 0 & 0 & 0 & 3 & 0 & 0 & 0 & 0 & 3 & 0 & 0 & 0 & 0 \\ 0 & 3 & 0 & 0 & 0 & 0 & 3 & 0 & 0 & 0 & 0 & 3 & 0 & 0 & 0 & 0 & 3 & 0 & 0 & 0 \\ 0 & 0 & 3 & 0 & 0 & 0 & 0 & 3 & 0 & 0 & 0 & 0 & 3 & 0 & 0 & 0 & 0 & 3 & 0 & 0 \\ 0 & 0 & 0 & 3 & 0 & 0 & 0 & 0 & 3 & 0 & 0 & 0 & 0 & 3 & 0 & 0 & 0 & 0 & 3 & 0 \\ 0 & 0 & 0 & 0 & 3 & 0 & 0 & 0 & 0 & 3 & 0 & 0 & 0 & 0 & 3 & 0 & 0 & 0 & 0 & 3\end{array}\right]$

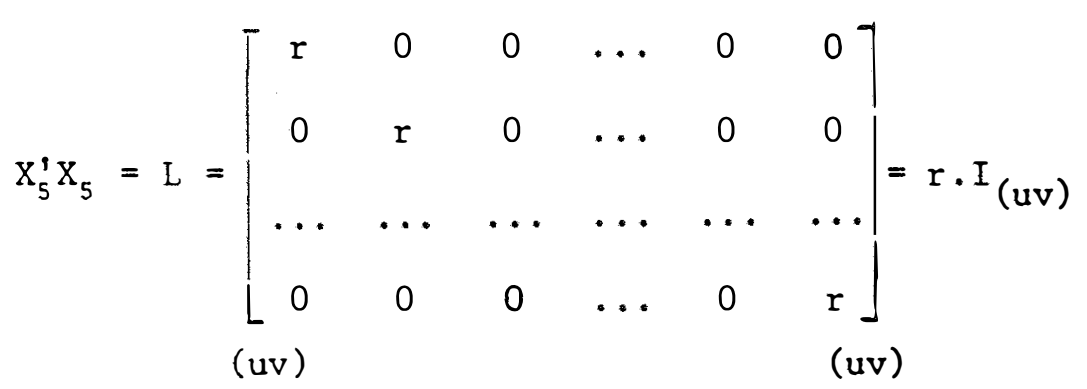

é a matriz diagonal associada ao número de repetições dos pares $\left(t t^{\prime}\right)_{15}$

Obteve-se, então, o sistema de equações normais:

$\left[\begin{array}{lllll}\mathrm{N} & \mathrm{x} & \mathrm{y} & \mathrm{z} & \mathrm{w} \\ \mathrm{x}^{\prime} & \mathrm{R} & \mathrm{N} & \mathrm{P} & \mathrm{S} \\ \mathrm{y}^{\prime} & \mathrm{N}^{\prime} & \mathrm{A} & \mathrm{K} & \mathrm{V} \\ \mathrm{z}^{\prime} & \mathrm{P}^{\prime} & \mathrm{K}^{\prime} & \mathrm{U} & \mathrm{H} \\ \mathrm{W}^{\prime} & \mathrm{S}^{\prime} & \mathrm{V}^{\prime} & \mathrm{H}^{\prime} & \mathrm{L}\end{array}\right]\left[\begin{array}{c}\hat{\mathrm{m}} \\ \hat{\tau} \\ \hat{\mathrm{\tau}} \\ \hat{\delta}\end{array}\right]=\left[\begin{array}{c}\mathrm{X}_{1}^{\prime} \mathrm{Y} \\ \mathrm{X}_{2}^{\prime} \mathrm{Y} \\ \mathrm{X}_{3}^{\prime} \mathrm{Y} \\ \mathrm{X}_{4}^{\prime} \mathrm{Y} \\ \mathrm{X}_{5}^{\prime} \mathrm{Y}\end{array}\right]=\left[\begin{array}{c}\mathrm{G} \\ \mathrm{T} \\ \mathrm{B} \\ \mathrm{T} \\ \Delta\end{array}\right]$

onde,

$\hat{\mathrm{m}}=$ efeito estimado da média geral;

$G=$ total geral observado; 


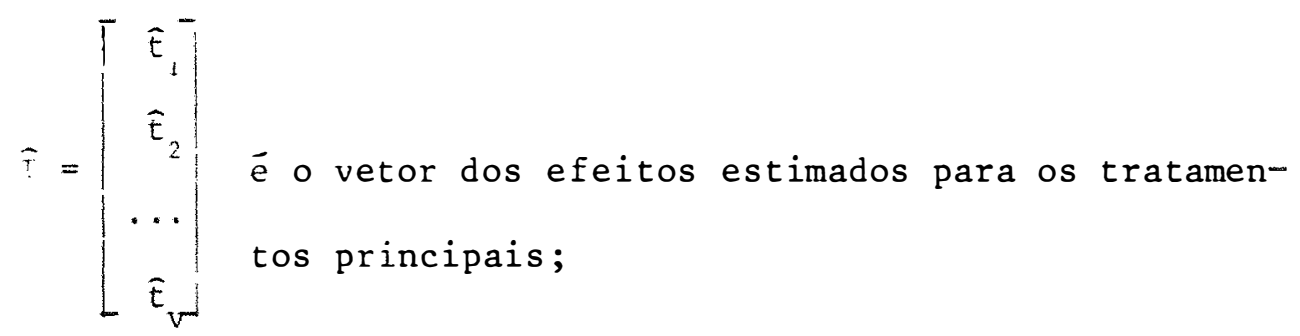

(v) (1)

$T=\left[\begin{array}{c}\mathrm{T}_{1} \\ \mathrm{~T}_{2} \\ \cdots \\ \mathrm{T}_{\mathrm{V}}\end{array}\right]$ é o vetor dos totais observados para os tratamen-

(v) (1)

$\hat{B}=\left[\begin{array}{c}b_{1} \\ b_{2} \\ \ldots \\ b_{a}\end{array}\right]$ é o vetor dos efeitos estimados para blocos;

(a) (1)

$B=\left[\begin{array}{c}B_{1} \\ B_{2} \\ \ldots \\ B_{a}\end{array}\right]$ é o vetor dos totais observados para blocos;

(a) (1)

$\hat{\tau}^{*}=\left[\begin{array}{r}\hat{t}_{i}^{\prime} \\ \hat{t}_{2}^{\prime} \\ \cdots \\ \hat{t}_{u}^{\prime}\end{array}\right]$ tos secundärios;

(u) (1) 
48.

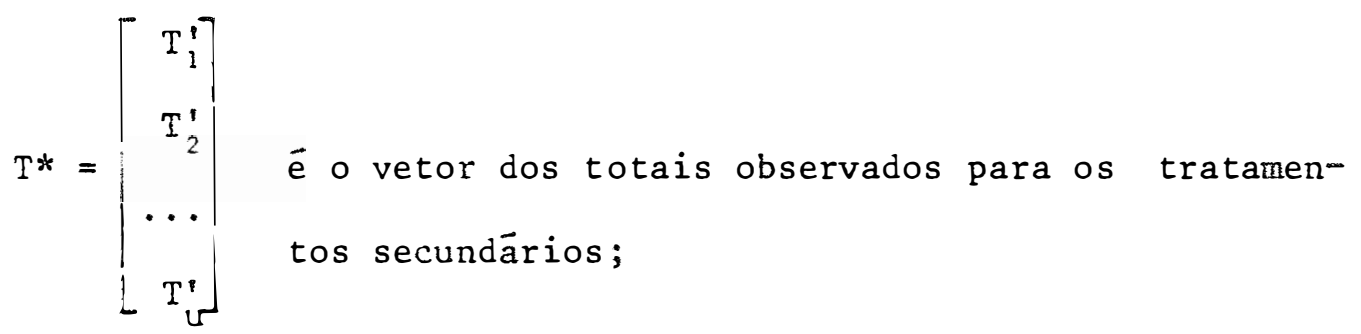
(u) (I)

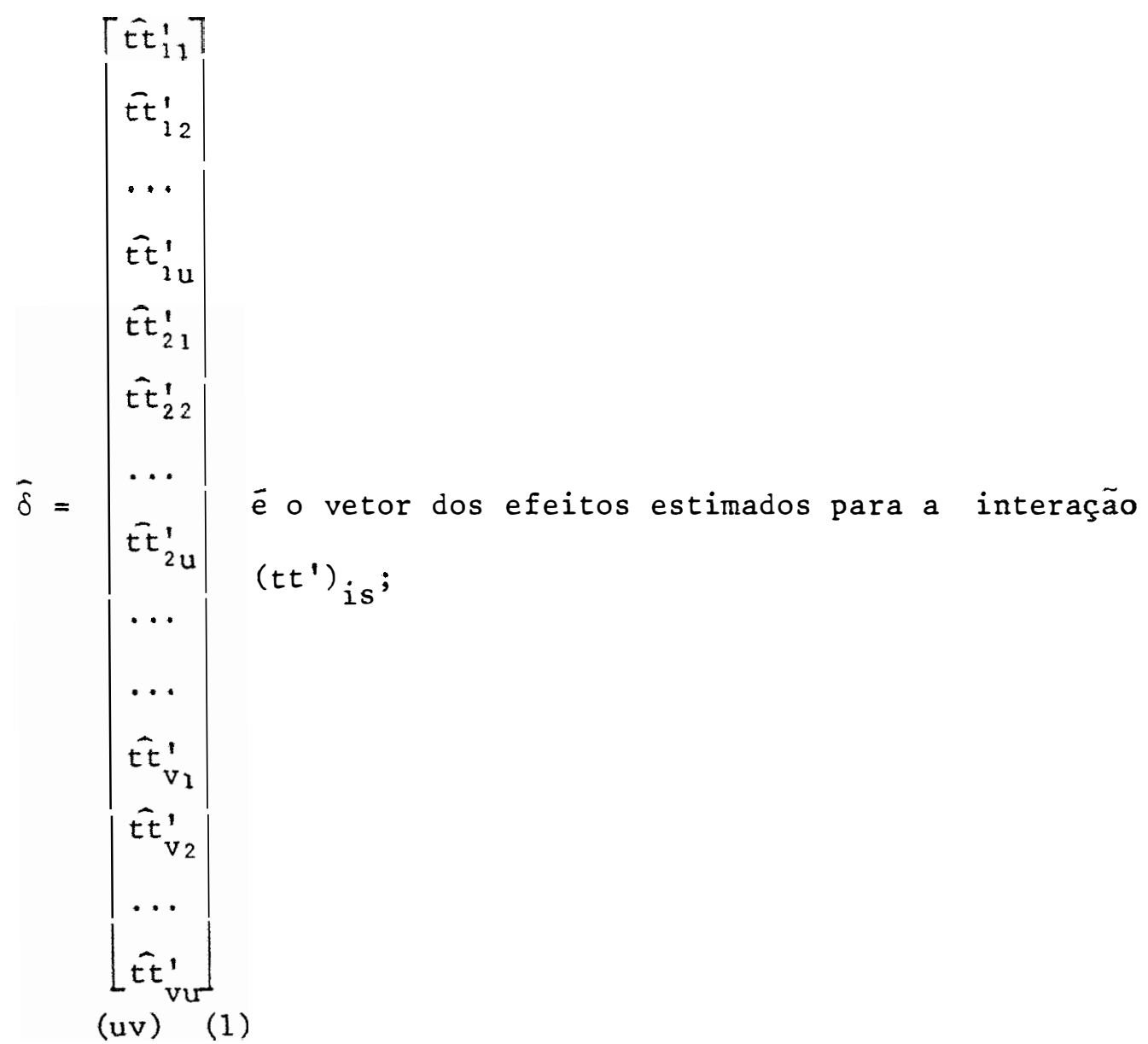


49.

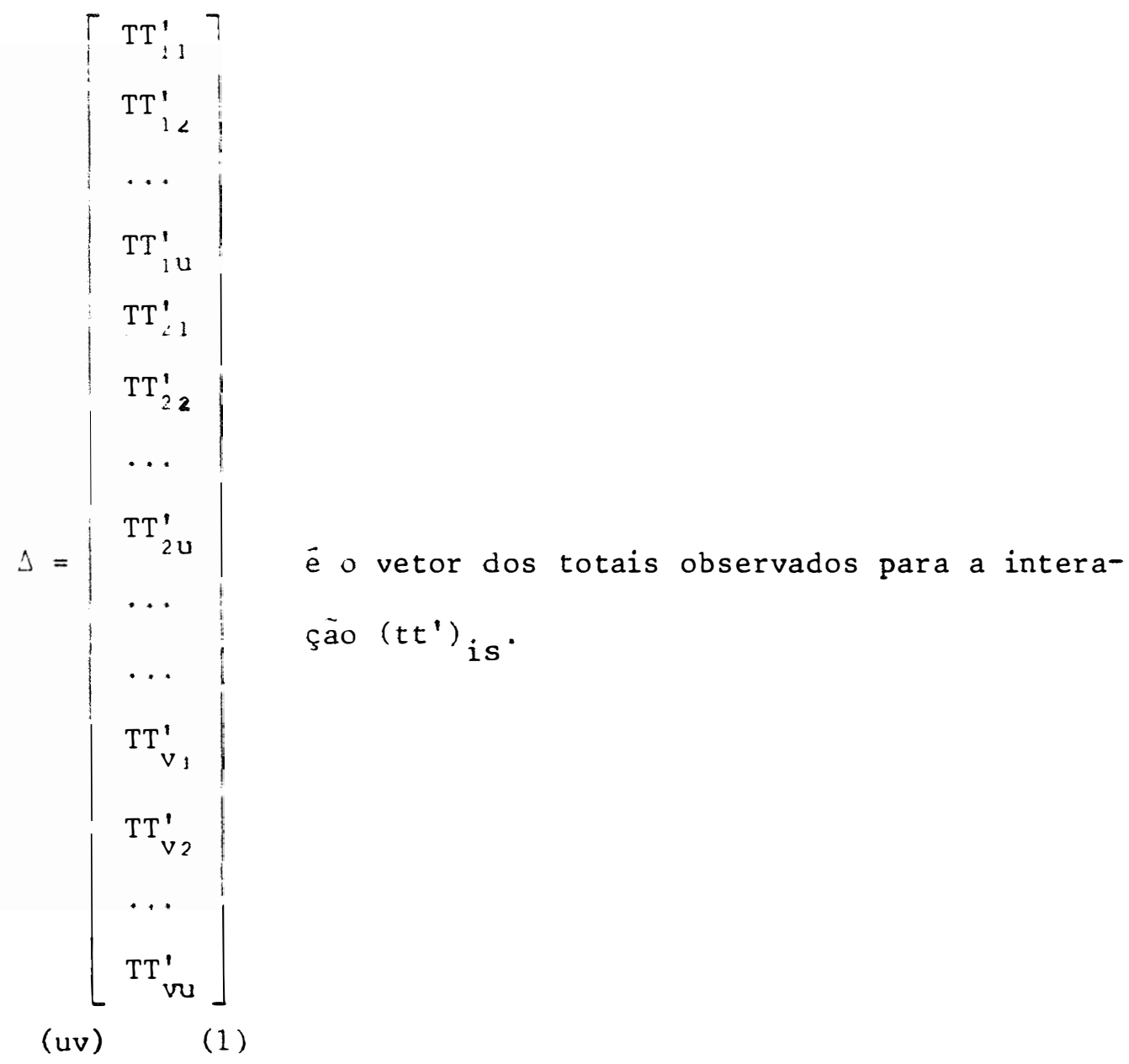

4.4 - Solução do Sistema

Efetuando-se as multiplicações sugeridas em (a.1) fo ram obtidas as equaçōes:

$$
\begin{aligned}
& n \hat{m}+x \hat{\tau}+y \hat{\beta}+z^{*}+w \hat{\delta}=G \\
& x^{\prime} \hat{m}+R \hat{\tau}+N \hat{\beta}+P \hat{\tau}^{*}+S \hat{\delta}=T \\
& y^{\prime} \hat{m}+N^{\prime} \hat{\tau}+A \hat{\beta}+K \hat{\tau}^{*}+V \hat{\delta}=B \\
& z^{\prime} \hat{m}+P^{\prime} \hat{\tau}+K^{\prime} \hat{\beta}+U \hat{\tau}^{*}+H \hat{\delta}=T^{*} \\
& w^{\prime} \hat{m}+S^{\prime} \hat{\tau}+V^{\prime} \hat{\beta}+H^{\prime} \hat{\tau}^{*}+L \hat{\delta}=\Delta
\end{aligned}
$$


No entanto, face às restrições impostas em (4.2) e às estruturas das matrizes envolvidas, conforme discutido em (4.3), resultou:

$$
\begin{array}{ll}
\mathrm{n} \hat{\mathrm{m}} & =\mathrm{G} \\
\mathrm{x}^{\prime} \hat{\mathrm{m}}+\mathrm{R} \hat{\tau}+\mathrm{N} \hat{\beta} & =\mathrm{T} \\
\mathrm{y}^{\prime} \hat{\mathrm{m}}+\mathrm{N}^{\prime} \bar{\tau}+\mathrm{A} \bar{B} & =\mathrm{B} \\
\mathrm{z}^{\prime} \hat{\mathrm{m}} & =\mathrm{U} \hat{\tau}^{*} \\
\mathrm{w}^{\prime} \hat{\mathrm{m}}+\mathrm{S}^{\prime} \bar{\tau}+\mathrm{V}^{\prime} \hat{\beta}+\mathrm{H}^{\prime} \hat{\tau}^{*}+\mathrm{L}^{*} & =\Delta
\end{array}
$$

Então, de (1) obteve-se

$$
\widehat{\mathrm{m}}=\frac{\mathrm{G}}{\mathrm{n}}=\frac{\mathrm{G}}{\mathrm{urv}}
$$

Por sua vez, (2) e (3) comportaram-se de modo análogo ao sistema de equações para blocos incompletos, discutido por PI MENTEL GOMES (1967), resultando:

$$
\operatorname{de}(3) \Longrightarrow \hat{B}=A^{-1} B-A^{-1} N^{\prime} \hat{\tau}-A^{-1} y^{\prime} \hat{m} \quad, \quad(\alpha * *)
$$

e, substituindo-se $\hat{B}$ em (2), obteve-se

$$
\begin{gathered}
R \bar{\tau}=T-N A^{-1} B+N A^{-1} N^{\prime} \hat{\tau}+N A^{-1} y^{\prime} \hat{m}-x^{\prime} \hat{m} \\
\therefore \quad\left(R-N A^{-1} N^{\prime}\right) \hat{\tau}=T-N A^{-1} B+\left(N A^{-1} y^{\prime}-x^{3}\right) \hat{m} \\
\text { Mas, desenvolvendo-se segundo (4.3) obteve-se } \\
N A^{-1} y^{\prime}=x^{\prime}
\end{gathered}
$$

resultando, então, o sistema de equações normais para efeitos esti- 
mados dos tratamentos principais, ajustados para efeitos de blocos:

$$
\left(R-N A^{-1} N^{\prime}\right) \bar{\tau}=T-N A^{-1} B \text {. }
$$

Fazendo-se, como em PIMENTEL GOMES (1967) e RAGHAVA-

RAO (1971)

$$
R-N A^{-i} N^{\prime}=C \quad \text { e } \quad T-N A^{-1} B=Q
$$

onde $C$ tem dimensões (v) x (v) e ë tal que

$$
c_{i i}{ }^{\prime}= \begin{cases}\operatorname{ur}\left(1-\frac{1}{k}\right) & , \text { se } i=i^{\prime} \\ -\frac{\lambda u}{k}, & \text { se } i \neq i^{\prime}\end{cases}
$$

e Q ë um vetor de dimensões (v) x (1), tal que

$$
Q_{i}=T_{i}-\frac{1}{k} J_{i}
$$

com $\mathrm{J}_{i}$ sendo a soma dos totais dos blocos que contêm o i-ésimo tratamento principal, obteve-se o sistema de equações normais como de modo usual na teoria dos blocos incompletos:

$$
C \widehat{T}=Q \quad
$$

Notadamente, C é uma matriz singular de característi ca $\hat{\imath}=\mathrm{v}-1$. Desse modo, adotou-se uma matriz $Z$, de restrições, conforme PIMENTEL GOMES (1967), tal que:

$$
z \bar{\tau}=\phi \quad \text { e } \quad z=-\frac{\lambda u}{k} \underset{(v)(v)}{E}
$$

e obteve-se o sistema $M \widehat{\tau}=Q$, onde $M=C-Z$ é do tipo 
52.

$$
m_{i i^{\prime}}=\left\{\begin{array}{cc}
\frac{\lambda u v}{k}, & \text { se } i=i^{\prime}, \\
0, & \text { se } i \neq i^{\prime},
\end{array}\right.
$$

pois :

$$
\begin{aligned}
i=i^{\prime} \Longrightarrow m_{i i} & =u r\left(1-\frac{1}{k}\right)+\frac{\lambda u}{k}=\frac{u r k-u r}{k}+\frac{\lambda u}{k} \\
& =\frac{u r(k-1)}{k}+\frac{\lambda u}{k}
\end{aligned}
$$

Mas, nos blocos incompletos balanceados $r(k-1)=\lambda(v-1)$

$$
\begin{aligned}
& \therefore \quad m_{i i}=\frac{u \lambda v-u \lambda+u \lambda}{k} \quad \therefore m_{i i}=\frac{\lambda u v}{k} \\
& i \neq i^{\prime} \Longrightarrow m_{i i^{\prime}}=-\frac{\lambda u}{k}+\frac{\lambda u}{k}=0
\end{aligned}
$$

então

$$
M=\frac{\lambda u v}{k} I_{(v)}
$$

e, desse modo,

$$
M^{-1}=\frac{k}{\lambda u v} I_{(v)} \text {. }
$$

Assim, uma solução para $C \hat{\tau}=Q$ pode ser obtida de

$$
\widehat{\tau}=M^{-1} Q
$$

Retomando-se o sistema dado em $(\alpha .3)$ e resolvendo-se

(4), obteve-se

$$
\hat{\tau}^{*}=U^{-1} T^{*}-U^{-1} z^{\prime} \hat{m}
$$




$$
\therefore \quad \hat{\mathrm{t}}_{\mathrm{s}}^{\prime}=\frac{1}{\mathrm{rv}} \mathrm{T}_{\mathrm{s}}^{*}-\hat{\mathrm{m}},
$$

que é uma solução idêntica àquelas obtidas para tratamentos secundá rios nos ensaios em parcelas subdivididas com delineamentos em blocos (completos) casualizados.

Para a determinação dos efeitos estimados dos pares tt'is, foram substituidos em (5) o valor

$$
\widehat{\tau}=R^{-1} T-R^{-1} N \widehat{\beta}-R^{-1} x^{\prime} \hat{m},
$$

obtido de (2), e os valores de $\bar{\beta}$ e $\widehat{\tau}^{*}$, obtidos respectivamente de $(\alpha . *)$ e $(\alpha .13)$, resultando:

$$
\begin{aligned}
W^{\prime} \hat{m} & +S^{\prime}\left(R^{-1} T-R^{-1} N \hat{B}-R^{-1} x^{\prime} \hat{m}\right)+V^{\prime}\left(A^{-1} B-A^{-1} N^{\prime} \hat{\tau}-A^{-1} y^{\prime} \hat{m}\right)+ \\
& +H^{\prime}\left(U^{-1} T^{*}-U^{-1} z^{\prime} \hat{m}\right)+L \hat{\delta}=\Delta,
\end{aligned}
$$

então

$$
\begin{aligned}
W^{\prime} \hat{m} & +S^{\prime} R^{-1} T-S^{\prime} R^{-1} N\left(A^{-1} B-A^{-1} N^{\prime} \bar{\tau}-A^{-1} y^{\prime} \hat{m}\right)-S^{\prime} R^{-1} x^{\prime} \hat{m}+ \\
& +V^{\prime} A^{-1} B-V^{\prime} A^{-1} N^{\prime} \hat{\tau}-V^{\prime} A^{-1} y^{\prime} \hat{m}+H^{\prime} U^{-1} T^{*}-H^{\prime} U^{-1} z^{\prime} \hat{m}+ \\
& +L \hat{\delta}=\Delta
\end{aligned}
$$

e

$$
\begin{aligned}
w^{\prime} \hat{m} & +S^{\prime} R^{-1} T-S^{\prime} R^{-1} N A^{-1} B+S^{\prime} R^{-1} N A^{-1} N^{\prime} \hat{\tau}+S^{\prime} R^{-1} N A^{-1} y^{\prime} \hat{m}- \\
& -S^{\prime} R^{-1} x^{\prime} \hat{m}+V^{\prime} A^{-1} B-V^{\prime} A^{-1} N^{\prime} \hat{\tau}-V^{\prime} A^{-1} y^{\prime} \hat{m}+H^{\prime} U^{-1} T^{*}- \\
& -H^{\prime} U^{-1} z^{\prime} \hat{m}+L^{\hat{\delta}}=\Delta \\
\therefore \quad \widehat{\delta}=L^{-1} \Delta & -L^{-1} S^{\prime} R^{-1} T+L^{-1}\left(S^{\prime} R^{-1} N-V^{\prime}\right) A^{-1} B-L^{-1} H^{\prime} U^{-1} T^{*}+ \\
& +L^{-1}\left(V^{\prime} A^{-1}-S^{\prime} R^{-1} N A^{-1}\right) N^{\prime} \hat{\tau}+ \\
& +L^{-1}\left(S^{\prime} R^{-1} x^{\prime}+H^{\prime} U^{-1} z^{\prime}+V^{\prime} A^{-1} y^{\prime}-S^{\prime} R^{-1} N A^{-1} y^{\prime}-w^{\prime}\right) \hat{m} .
\end{aligned}
$$


Mas

$$
V^{\prime} A^{-1}=S^{\prime} R^{-1} N A^{-1} \quad \therefore \quad V^{\prime} A^{-1} y^{\prime}=S^{\prime} R^{-1} N A^{-1} y^{\prime}
$$

e

$$
\begin{gathered}
S^{\prime} R^{-1} x^{\prime}=H^{\prime} U^{-1} z^{\prime}=y^{\prime} \\
\therefore \quad S^{\prime} R^{-1} x^{\prime}+H^{\prime} U^{-1} z^{\prime}+V^{\prime} A^{-1} y^{\prime}-S^{\prime} R^{-1} N A^{-1} y^{\prime}-w^{\prime}=w^{\prime}
\end{gathered}
$$

entào

$$
\hat{\delta}=L^{-1} \Delta-L^{-1} S^{\prime} R^{-1} T-L^{-1} H^{\prime} U^{-1} T^{*}+L^{-1} w^{\prime} \hat{m} \quad(\alpha .15)
$$

onde,

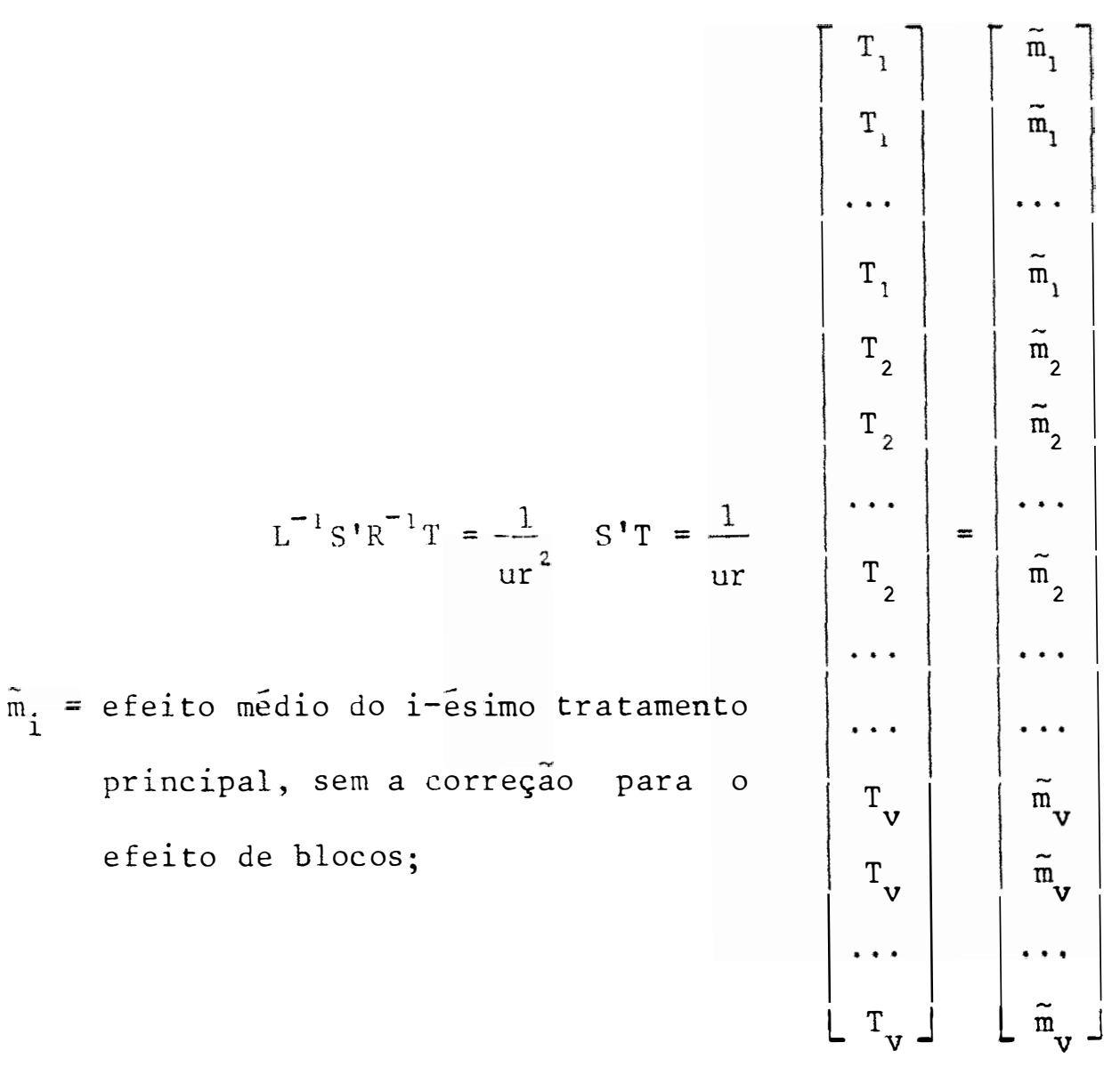

$$
\begin{aligned}
& \text { (uv) (1) (uv) (1) }
\end{aligned}
$$


55.

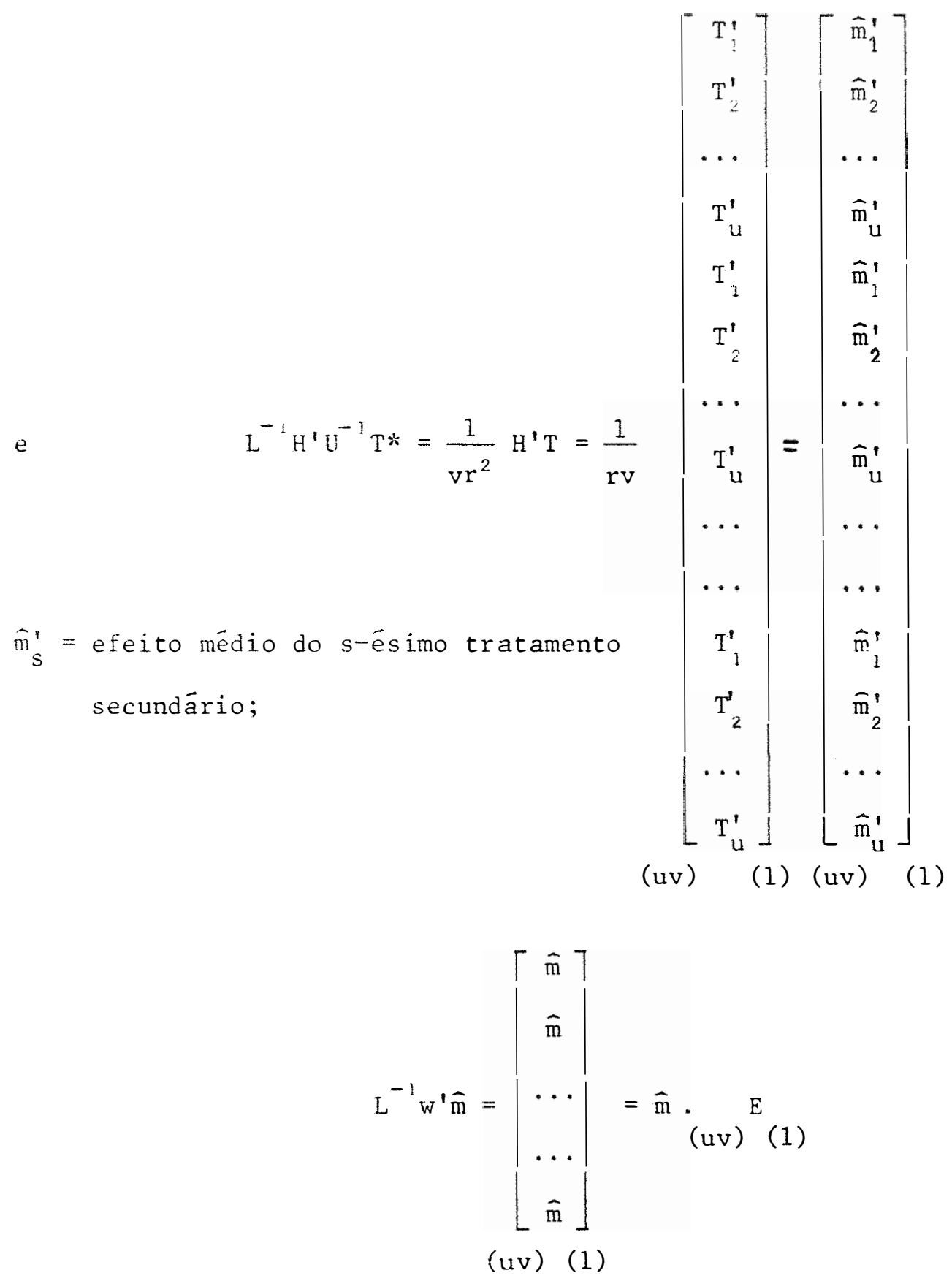


56.

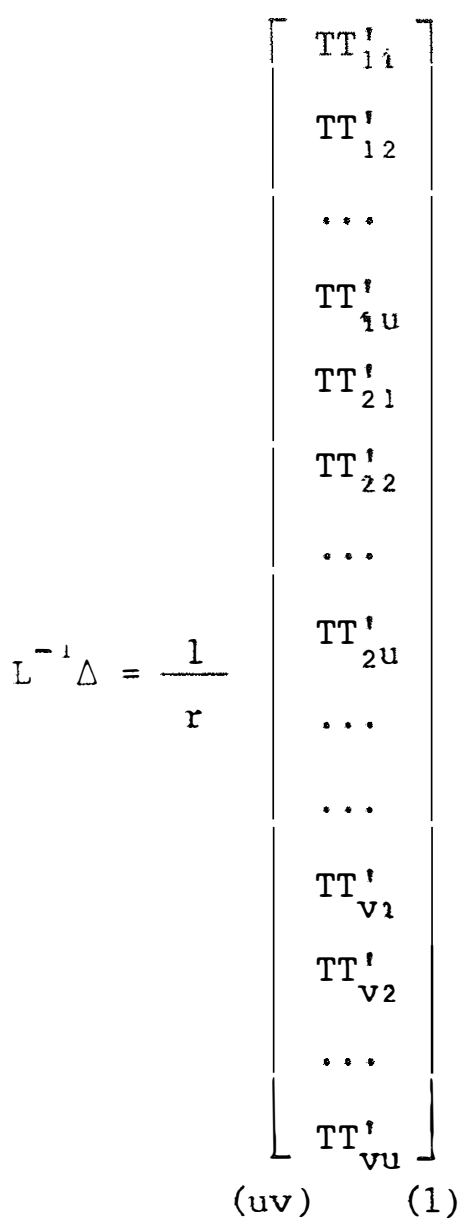


desse modo, de $(\alpha .15)$, obteve-se

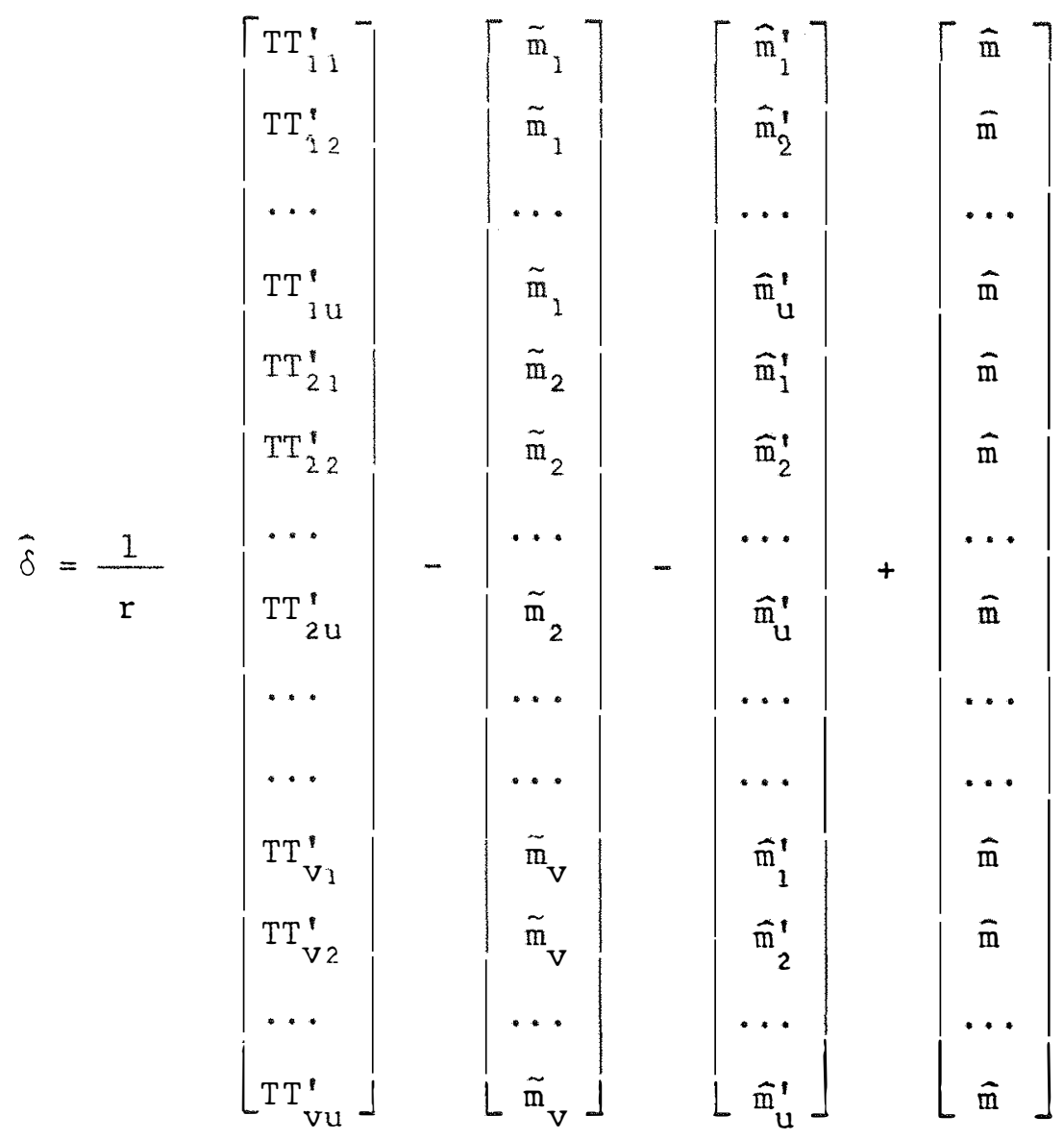

Assim, o efeito estimado para um elemento qualquer

tt! ficou

$$
\hat{\delta}_{i s}=\left(t t^{\prime}\right)_{i s}=\frac{T T_{i s}^{\prime}}{r}-\tilde{m}_{i}-\hat{m}_{s}^{\prime}+\hat{m} .
$$

Mas

$$
\tilde{m}_{i}=\hat{m}+\tilde{t}_{i} \quad \text { e } \quad \hat{m}_{s}^{\prime}=\hat{m}+t_{s}^{\prime}
$$

onde $\tilde{t}_{i} \bar{e}$ o efeito estimado do i-ésimo tratamento principal, obtido 
58.

de modo usual (sem o ajuste para efeitos de blocos)

$$
\begin{aligned}
& \therefore \hat{\delta}_{i s}=\frac{T T_{i s}^{\prime}}{r}-\hat{m}-\tilde{t}_{i}-\hat{m}-\hat{t}_{s}^{\prime}+\hat{m} \\
& \therefore \hat{\delta}_{i s}=\frac{T T_{i s}^{\prime}}{r}-\hat{m}-\tilde{t}_{i}-\hat{t}_{s}^{\prime}
\end{aligned}
$$

onde $\tilde{\mathrm{t}}_{i}=\frac{\mathrm{T}_{\mathrm{i}}}{u r}-\hat{\mathrm{m}}$, que é um resultado idêntico àqueles obtidos para efeitos estimados dos elementos $\left(t t^{\prime}\right)_{\text {is }}$ em ensaios com parcelas subdivididas, envolvendo delineamentos em blocos (completos) casualizados .

4.5 - Dispersão

Considerando-se as pressuposições do modelo abordado, relatadas em (4.2) com base em estudos desenvolvidos por CHAKRABAR TI (1962), GILL (1978), LEAL (1979) e DINIZ (1980), entre outros,ob teve-se: 


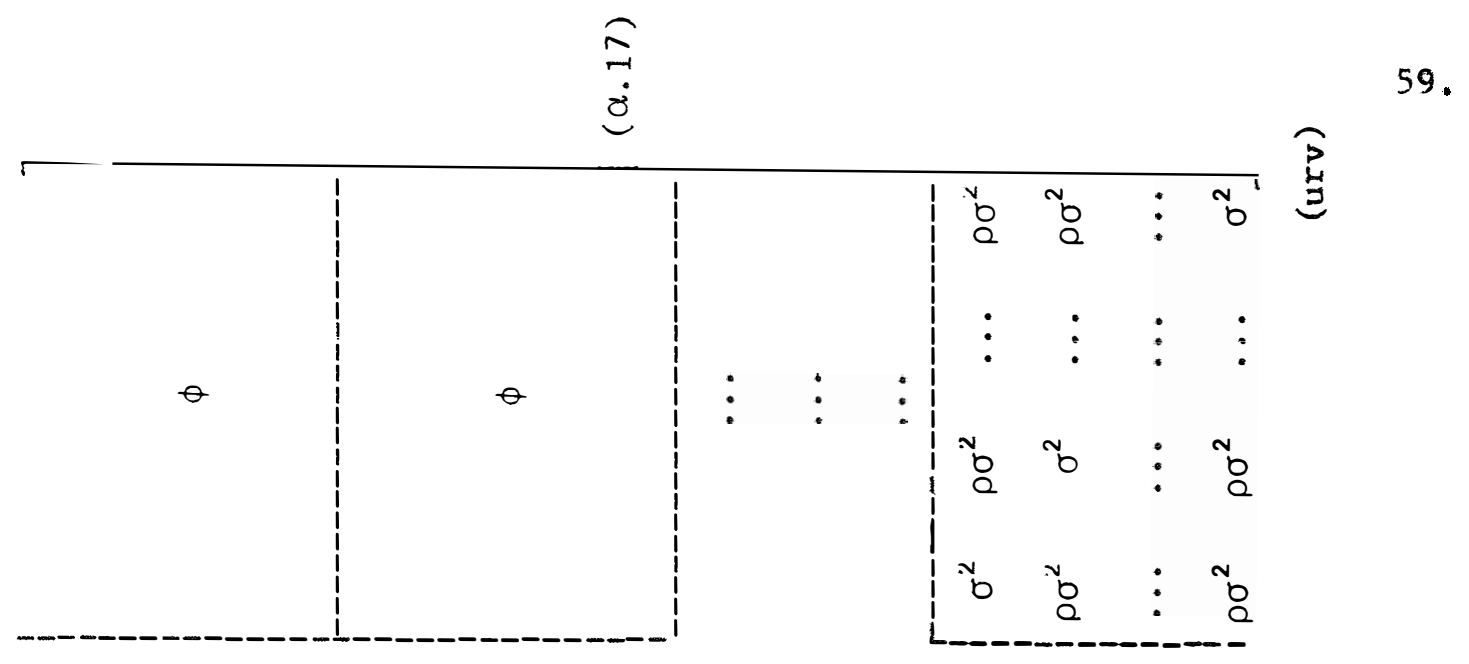

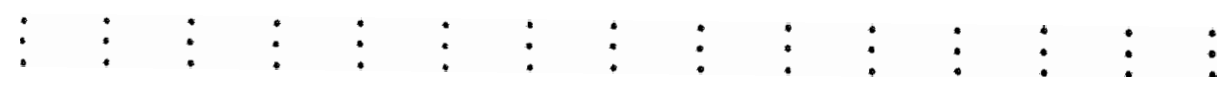

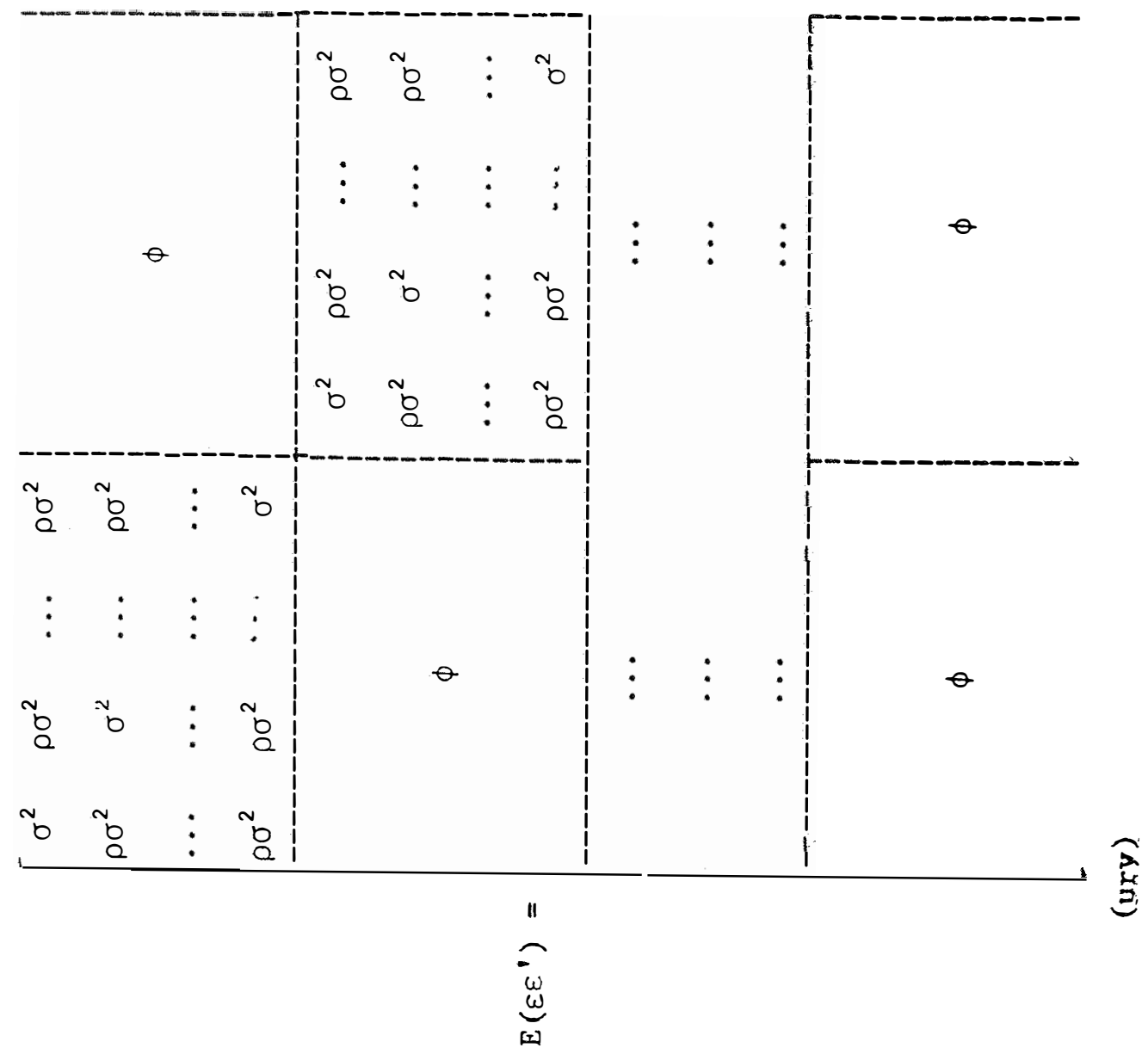


Então, se o modelo é adequado, existem ak = rv submatrizes uniformes do tipo

$$
W=\left[\begin{array}{cccc}
\sigma^{2} & \rho \sigma^{2} & \cdots & \rho \sigma^{2} \\
\rho \sigma^{2} & \sigma^{2} & \cdots & \rho \sigma^{2} \\
\cdots & \cdots & \cdots & \cdots \\
\rho \sigma^{2} & \rho \sigma^{2} & \cdots & \sigma^{2}
\end{array}\right]
$$

(u)

(u)

na posição diagonal, e as demais submatrizes são todas nulas. Obvia mente, existe uma matriz $W$ para cada uma das rv parcelas, ou, ainda, uma matriz $W$ para cada tratamento de cada repetição.

\section{5 .1 - Dispersão para tratamentos principais: $D(\tilde{\tau})$}

Para a determinação da matriz de dispersão para tratamentos principais, considerou-se como de modo usual

$$
D(\hat{\tau})=E\left\{[\hat{\tau}-E(\hat{\tau})][\hat{\tau}-E(\hat{\tau})]^{\prime}\right\},
$$

onde, por $(\alpha .12), \hat{\tau}=M^{-1} Q$, e, por $(\alpha .6 .1), Q=T-N A^{-1} B$,

$$
\therefore \quad \bar{\tau}=M^{-1} T-M^{-1} N A^{-1} B .
$$

$$
\text { Mas, por } \begin{aligned}
(\alpha .1), T & =X_{2}^{\prime} Y \\
\therefore T & =X_{2}^{\prime}(X \theta+\varepsilon)
\end{aligned}
$$

e

$$
\begin{aligned}
B & =X_{3}^{1} Y \\
\therefore \quad B & =X_{3}^{\prime}(X \theta+\varepsilon)
\end{aligned}
$$


entào, de $(\alpha .19)$, obteve-se

$$
\widehat{\tau}=M^{-1} X_{2}^{\prime} X \theta+M^{-1} X_{2}^{\prime} \varepsilon-M^{-1} N A^{-1} X_{3}^{\prime} X \theta-M^{-1} N A^{-1} X_{3}^{\prime} \varepsilon
$$

e, desse modo

$$
\begin{array}{r}
E(\widehat{\tau})=M^{-1} X_{2}^{1} X \theta-M^{-1} N A^{-1} X_{3}^{1} X \theta \\
\therefore \quad \bar{\tau}-E(\widehat{\tau})=M^{-1}\left(X_{2}^{\prime}-N A^{-1} X_{3}^{\prime}\right) \varepsilon
\end{array}
$$

e, fazendo-se $F=X_{2}^{1}-N^{-1} X_{3}^{1}$, obteve-se

$$
\begin{gathered}
\hat{\tau}-E(\widehat{\tau})=M^{-1} F \varepsilon \\
\therefore \quad D(\widehat{\tau})=M^{-1} F E\left(\varepsilon \varepsilon^{\prime}\right) F^{\prime} M^{-1}
\end{gathered}
$$

Mas, por $(\alpha .11), M^{-1}=\frac{k}{\lambda u v} I_{(v)}$

$$
\therefore D(\bar{\tau})=\frac{k^{2}}{\lambda^{2} u^{2} v^{2}} F E\left(\varepsilon \varepsilon^{\prime}\right) F^{\prime} .
$$

Para melhor interpretar o produto $F E\left(\varepsilon \varepsilon^{\prime}\right) F^{\prime}$, construiu-se a matriz $F=X_{2}^{\prime}-N^{-1} X_{3}^{\prime}$, relativa ao exemplo descrito em 4.1. Essa matriz é apresentada a seguir, com partições a nível de subparcelas.

Recorrendo-se, então, ao exemplo de (4.1), onde usou- se

$$
\begin{aligned}
& v=4 \text { tratamentos principais; } \\
& \mathrm{u}=5 \text { tratamentos secundários; } \\
& \mathrm{a}=6 \text { blocos; } \\
& \mathrm{k}=2 \text { tratamentos principais por bloco; } \\
& \mathrm{r}=3 \text { repetições para cada tratamento principal, }
\end{aligned}
$$

obteve-se a seguinte matriz $\mathrm{F}$ 


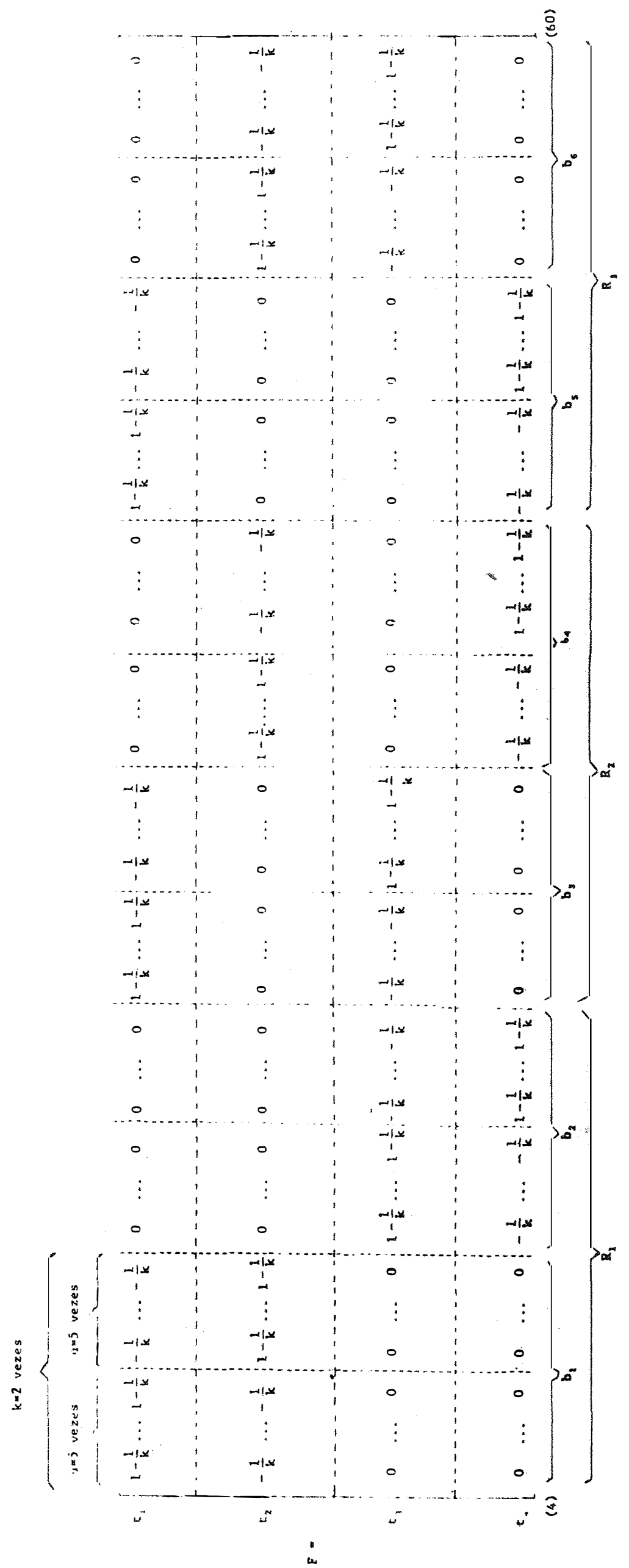

62. 
Efetuando-se, então, o produto $E\left(\varepsilon \varepsilon^{\prime}\right)$ F, obteve-se a pröpria matriz F, a menos de uma constante:

$$
E\left(\varepsilon \varepsilon^{\prime}\right) F=[1+(u-1) \rho] \sigma^{2} \cdot F
$$

então, de $(\alpha .20)$, resultou

$$
D(\hat{\tau})=\frac{k^{2}}{\lambda^{2} u^{2} v^{2}}[1+(u-1) \rho] \sigma^{2} F F^{\prime}
$$

Mas,

$$
\begin{aligned}
F F^{\prime} & =\left(X_{2}^{\prime}-N A^{-1} X_{3}^{\prime}\right)\left(X_{2}-X_{3} A^{-1} N^{\prime}\right) \\
& =X_{2}^{\prime} X_{2}-X_{2}^{\prime} X_{3} A^{-1} N^{\prime}-N A^{-1} X_{3}^{\prime} X_{2}+N A^{-1} X_{3}^{\prime} X_{3} A^{-1} N^{\prime}
\end{aligned}
$$

onde, por (4.3)

$$
\begin{aligned}
& x_{2}^{\prime} x_{2}=R \\
& x_{2}^{\prime} x_{3}=N \quad \therefore \quad\left(x_{2}^{\prime} x_{3}\right)^{\prime}=x_{3}^{\prime} x_{2}=N^{\prime} \\
& x_{3}^{\prime} x_{2}=N^{\prime} \\
& x_{3}^{\prime} x_{3}=A
\end{aligned}
$$

então

$$
\begin{aligned}
& F F^{\prime}=R-N A^{-1} N^{\prime}-N A^{-1} N^{\prime}+N A^{-1} A A^{-1} N^{\prime} \\
& \therefore F F^{\prime}=R-N A^{-1} N^{\prime}=C \text {, por }(\alpha .6 .1) .
\end{aligned}
$$

Desse modo, de ( $\alpha .21)$, obteve-se

$$
D(\bar{\tau})=\frac{k^{2}}{\lambda^{2} u^{2} v^{2}}[1+(u-1) \rho] \sigma^{2} \cdot C
$$

ou, reportando-se a $(\alpha .11)$

$$
D(\hat{\tau})=M^{-1} \mathrm{CM}^{-1}[1+(\mathrm{u}-1) \rho] \sigma^{2},
$$


resultado que, a menos do valor $[1+(u-1) \rho]$, é idêntico àqueles ob tidos por CHAKRABARTI (1962), PIMENTEL GOMES (1967) e RAGHAVARAO (1971), dentre outros, para a matriz de dispersão dos tratamentos em delineamentos com blocos incompletos balanceados.

Entretanto, lembrando-se $(\alpha .7)$, obteve-se, para um elemento genérico di.i., de $D(\tilde{\tau})$

$$
d_{i . i^{\prime}}=\left\{\begin{array}{c}
\frac{k(v-1)}{\lambda u v^{2}}[1+(u-1) \rho] \sigma^{2}, \text { se } i=i^{\prime} \\
-\frac{k}{\lambda u v^{2}}[1+(u-1) \rho] \sigma^{2}, \text { se } i \neq i^{\prime}
\end{array}\right.
$$

pois,

$$
\text { a) } i=i^{\prime} \Longrightarrow d_{i . i .}=\frac{k^{2}}{\lambda^{2} u^{2} v^{2}} \frac{u r(k-1)}{k}[1+(u-1) \rho] \sigma^{2}
$$

mas, $r(k-1)=\lambda(v-1)$,

$$
\therefore d_{\text {i.i. }},=\frac{k(v-1)}{\lambda u v^{2}}[1+(u-1) \rho] \sigma^{2}
$$

b) $i \neq i^{\prime} \Longrightarrow d_{i i^{\prime}}=\frac{k^{2}}{\lambda^{2} u^{2} v^{2}} \cdot \frac{-\lambda u}{k_{m}}[1+(u-1) \rho] \sigma^{2}$

$$
\therefore d_{i i^{\prime}}=-\frac{k}{\lambda u v^{2}}[1+(u-1) p] \sigma^{2}
$$

Assim, para a variância da diferença entre duas médias de efeitos estimados de tratamentos principais, observou-se

$$
\begin{aligned}
v\left(\hat{\mathrm{t}}_{i}-\hat{\mathrm{t}}_{i^{\prime}}\right) & =v\left(\hat{\mathrm{t}}_{i}\right)+\dot{v}\left(\hat{\mathrm{t}}_{i^{\prime}}\right)-2 \operatorname{Cov}\left(\hat{\mathrm{t}}_{i}, \hat{\mathrm{t}}_{i^{\prime}}\right) \\
& =\left[\frac{2 k(v-1)}{\lambda u v^{2}}+\frac{2 k}{\lambda u v^{2}}\right][1+(u-1) \rho] \sigma^{2}
\end{aligned}
$$




$$
\therefore V\left(\hat{E}_{i}-\bar{E}_{i},\right)=\frac{2 k}{\lambda u v}[1+(u-1) p] \sigma^{2}
$$

Naturalmente, resultados análogos foram obtidos usan do-se o conceito de função linear estimável, conforme abordado em (2.2.3). Então, por exemplo, para $i=1$ e $i^{\prime}=2$,

$$
\begin{gathered}
V\left(P^{\prime} \hat{\tau}\right)=[1,-1,0, \ldots, 0] \frac{k[1+(u-1) \rho] \sigma^{2}}{\lambda u v^{2}}\left[\begin{array}{ccccc}
\mathrm{v}-1 & -1 & \ldots & -1 & -1 \\
-1 & \mathrm{v}-1 & \ldots & -1 & -1 \\
\ldots & \ldots & \ldots & \ldots & \ldots \\
-1 & -1 & \ldots & -1 & \mathrm{v}-1
\end{array}\right]\left[\begin{array}{c}
1 \\
-1 \\
\ldots \\
0
\end{array}\right] \\
\therefore V\left(P^{\prime} \hat{\tau}\right)=\frac{2 k}{\lambda u v}\left[\begin{array}{ll}
1+(u-1) & 0
\end{array}\right] \sigma^{2} \\
\therefore
\end{gathered}
$$

e, usando-se os resultados obtidos por CHAKRABARTI (1962), COCHRAN e COX (1976) e GILL (1978), entre outros, bem como os resultados ob tidos neste estudo,em (4.6.5.1), que indicam como uma estimativa não tendenciosa para $[1+(u-1) \rho] \sigma^{2}$, o quadrado médio do Resíduo (a), obteve-se finalmente

$$
\hat{v}\left(\hat{t}_{i}-\hat{t}_{i},\right)=\frac{2 k}{\lambda u v} \operatorname{QMRes}(a),
$$

que é um resultado coerente com aqueles tradicionalmente encontrados na bibliografia, para variância entre duas médias de efeitos estimados de tratamentos em delineamentos em blocos incompletos balanceados:

$$
\hat{v}\left(\hat{E}_{i}-\hat{E}_{i \prime}\right)^{\prime}=\frac{2 k}{\lambda v} \operatorname{QMRes}(a)
$$


66.

4.5.2 - Dispersăo para tratamentos secundārios: $D\left(\bar{\tau}^{*}\right)$

$$
\begin{aligned}
& \text { Conforme }(4.5 .1) \text {, tomou-se } \\
& D\left(\widehat{\tau}^{*}\right)=E\left\{\left[\widehat{\tau}^{*}-E\left(\widehat{\tau}^{*}\right)\right]\left[\widehat{\tau}^{*}-E\left(\widehat{\tau}^{*}\right)\right]^{\prime}\right\}
\end{aligned}
$$

onde, por $(\alpha .13), \quad \hat{\tau} *=U^{-1} T^{*} * U^{-1} z^{\prime} \hat{m}$

$$
=\mathrm{U}^{-1} \mathrm{~T}^{*}-\widehat{\mathrm{m}} ; \mathrm{E}
$$

e por $(\alpha .1), \quad T^{*}=X_{4}^{1} Y=X_{4}^{1}(X \theta+\varepsilon)$, entáo,

$$
\begin{aligned}
& \widehat{\tau} *=U^{-1} X_{i_{4}}^{\prime} X \theta+U^{-1} X_{4_{k}}^{\prime} \varepsilon-\bar{m} \cdot E
\end{aligned}
$$

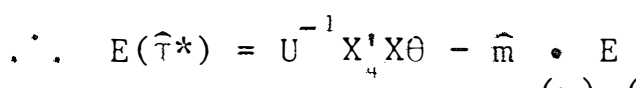

$$
\begin{aligned}
& \text { (u) (1) }
\end{aligned}
$$

e, desse modo,

$$
\begin{gathered}
\hat{\tau}^{*}-E\left(\hat{\tau}^{*}\right)=U^{-1} X_{4}^{\prime} \varepsilon . \\
\text { Assim, de }(\alpha .28) \text {, obteve-se } \\
D\left(\tau^{*}\right)=U^{-1} X_{4}^{\prime} E\left(\varepsilon \varepsilon^{\prime}\right) X_{4} U^{-1} \\
\therefore D\left(\tau^{*}\right)=\frac{1}{r^{2} v^{2}} X_{4}^{\prime} E\left(\varepsilon \varepsilon^{\prime}\right) X_{4} .
\end{gathered}
$$

Tomando-se como base o exemplo inicial, obteve-se

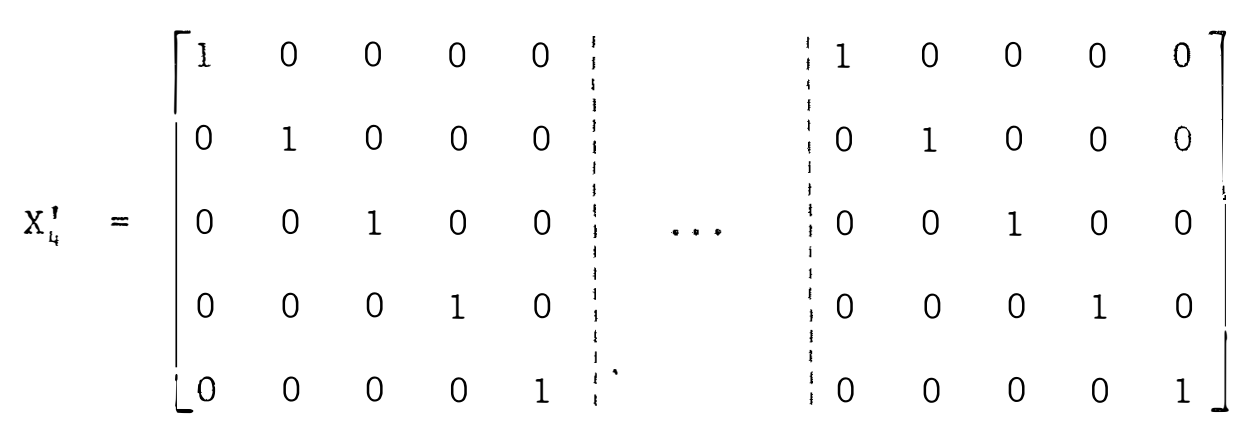


e, genericamente

$$
\left.\mathrm{X}_{4}^{i}=\mathrm{I}_{(\mathrm{u})} \mathrm{I}_{(\mathrm{u})} \mathrm{I}_{(\mathrm{u})}: \ldots \mathrm{I}_{(\mathrm{u})}\right]_{(\mathrm{urv})}
$$

donde

$$
X_{4}^{1} E\left(\varepsilon \varepsilon^{\prime}\right) X_{4}=r v\left[\begin{array}{cccc}
\sigma^{2} & \rho \sigma^{2} & \ldots & \rho \sigma^{2} \\
\rho \sigma^{2} & \sigma^{2} & \ldots & \rho \sigma^{2} \\
\ldots & \ldots & \ldots & \ldots \\
\rho \sigma^{2} & \rho \sigma^{2} & \ldots & \sigma^{2}
\end{array}\right]
$$

ou, por $(\alpha .18)$,

$$
X_{4}^{\prime} E\left(\varepsilon \varepsilon^{\prime}\right) X_{4}=r v ;{ }^{W}(u)
$$

Obteve-se, assim, de ( $(.29)$

$$
D\left(\hat{\tau}^{*}\right)=\frac{1}{r v} \dot{(u)}(u)
$$

com elementos

$$
d_{i i^{\prime}}^{*}= \begin{cases}\frac{\sigma^{2}}{r v}, & \text { se } i=i^{\prime} \\ \frac{\rho \sigma^{2}}{r v}, & \text { se } i \neq i^{\prime}\end{cases}
$$

E, para a variância da diferença entre duas médias de efeitos estimados de tratamentos secundārios, obteve-se

$$
V\left(\hat{E}_{i}^{\prime}-\hat{E}_{i}^{\prime}\right)=\frac{2}{r v}(1-\rho) \sigma^{2}
$$


e, usando-se os resultados obtidos por CHAKRABARTI (1962), COCHRAN e COX (1976) e GILL (1978), entre outros, bem como os resultados ob tidos neste estudo em (4.6.5.2), que indicam como uma estimativa não tendenciosa para ( $I-\rho) \sigma^{2}$, o quadrado médio do Resỉduo (b),

$$
V\left(\hat{t}_{i}^{\prime}-\hat{t}_{i}^{\prime},\right)=\frac{2}{r v} \operatorname{QMRes}(b)
$$

que é um resultado análogo àqueles encontrados tradicionalmente na literatura dos ensaios em parcelas subdivididas com delineamento em blocos (completos) casualizados, no tocante à variância estimada da diferença entre duas médias de efeitos estimados de tratamentos secundārios, conforme pode ser observado em (2.1.3).

4.5 .3 - Dispersão para a interação: $D(\bar{\delta})$

Tomando-se

$$
D(\widehat{\delta})=E\{[\hat{\delta}-E(\widehat{\delta})][\hat{\delta}-E(\widehat{\delta})] '\},
$$

onde, de $(\alpha .15)$,

$$
\begin{aligned}
\hat{\delta} & =L^{-1}\left(\Delta-S^{\prime} R^{-1} T-H^{\prime} U^{-1} T^{*}+w^{\prime} \hat{m}\right) \\
& =\frac{1}{r}\left(\Delta-\frac{1}{u r} S^{\prime} T-\frac{1}{r v} H^{\prime} T^{*}+\frac{1}{u r v} w^{\prime} G\right)
\end{aligned}
$$

e, como por $(\alpha .1)$

$$
\begin{aligned}
\Delta & =X_{5}^{\prime} Y \\
T & =X_{2}^{\prime} Y \\
T^{*} & =X_{4}^{\prime} Y \\
G & =X_{1}^{\prime} Y,
\end{aligned}
$$


ficou

$$
\hat{\delta}=\frac{1}{r}\left(X_{5}^{\prime}-\frac{1}{u r} S^{\prime} X_{2}^{\prime}-\frac{1}{r v} H^{\prime} X_{4}^{\prime}+\frac{1}{u r v} w^{\prime} X_{1}^{\prime}\right) Y
$$

então,

$$
\hat{\delta}-E(\bar{\delta})=\frac{1}{r}\left(X_{5}^{\prime}-\frac{1}{u r} S^{\prime} X_{2}^{\prime}-\frac{1}{r v} H^{\prime} X_{4}^{\prime}+\frac{1}{u r v} w^{\prime} X_{1}^{\prime}\right) \varepsilon
$$

e, fazendo-se

$$
\Gamma=X_{5}^{\prime}-\frac{1}{u r} S^{\prime} X_{2}^{\prime}-\frac{1}{r v} H^{\prime} X_{4}^{\prime}+\frac{1}{u r v} w^{\prime} X_{1}^{\prime}
$$

de $(\alpha .34)$, resultou

$$
\begin{aligned}
D(\widehat{\delta}) & =L^{-1} \Gamma E\left(\varepsilon \varepsilon^{\prime}\right) \Gamma^{\prime} L^{-1} \\
\therefore \quad D(\widehat{\delta}) & =\frac{1}{r^{2}} \Gamma E\left(\varepsilon \varepsilon^{\prime}\right) \Gamma^{\prime} .
\end{aligned}
$$

Para bem interpretar a matriz $\Gamma$, efetuou-se um estudo de suas componentes, com base no exemplo inicial. As generalizaf̧ões, em termos de número de tratamentos principais e tratamentos se cundários, resultaram como a seguir. No entanto, essa generalização foi apenas parcial, pois ficou "presa" ao esquema de blocos incompletos número 11.1 , de COCHRAN e COX (1976), utilizado no exemplo. Como é fácil ver, a outros esquemas correspondem outras constituif̧ões matriciais, sem que, no entanto, ocorra perda de generalidade, isto é, sem que se altere o resultado final.

$$
\text { Assim, }
$$




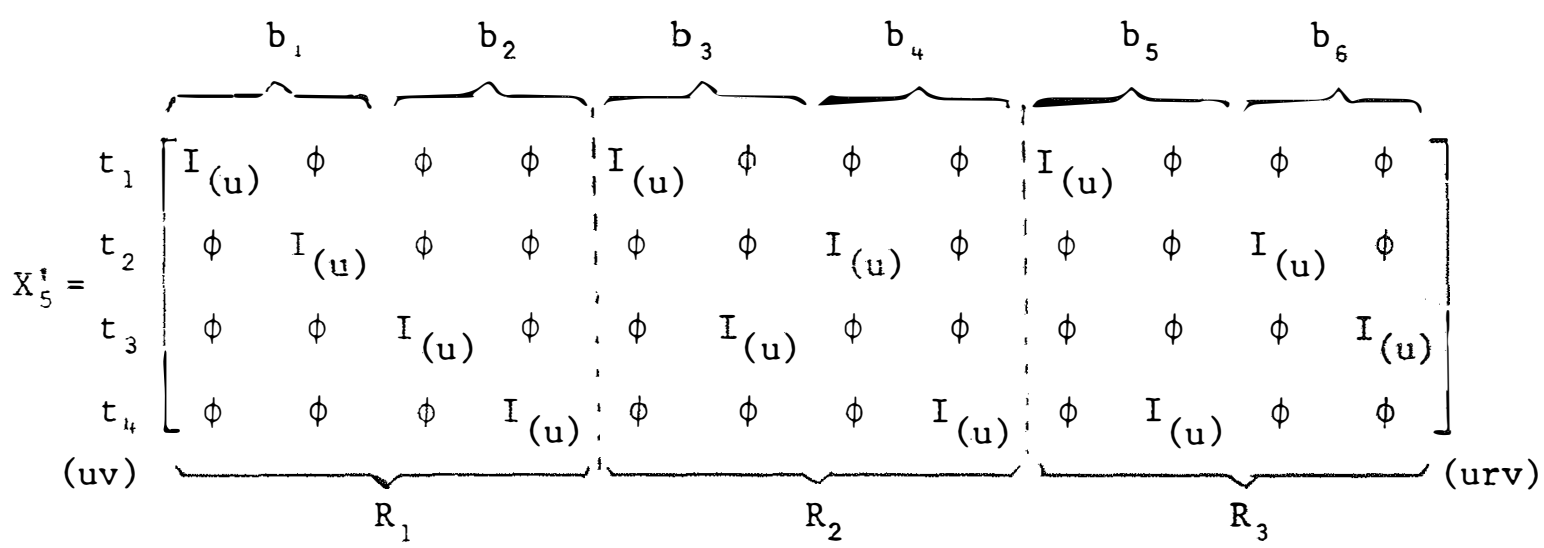

onde $\phi={ }_{(u)}^{\phi}(u)$

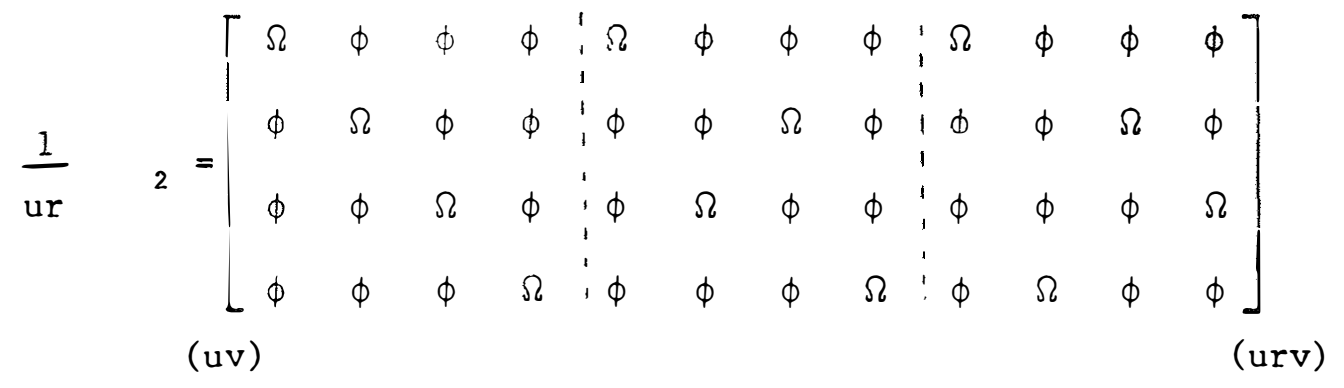

onde,

$$
\begin{gathered}
\Omega=\frac{1}{u} \dot{(u)}^{E}(\mathrm{u}) \\
\phi=\mathrm{Cu}^{\phi}(\mathrm{u}) \\
\frac{1}{\mathrm{rv}} \mathrm{H}^{\prime} \mathrm{X}_{4}^{\prime}=\left[\begin{array}{cccc}
\Psi & \Psi & \ldots & \Psi \\
\Psi & \Psi & \ldots & \Psi \\
\ldots & \ldots & \ldots & \ldots \\
\Psi & \Psi & \ldots & \Psi
\end{array}\right] \text { (urv) }
\end{gathered}
$$


onde $\Psi=\frac{1}{v} I_{(u)}$

$$
\frac{1}{u r v} w^{\prime} X_{i}^{\prime}=\frac{1}{u v} E_{(u v)}^{E}=\prod_{(u r v)}=
$$

Desse modo, a matriz $\Gamma$ ficou assim constituida:

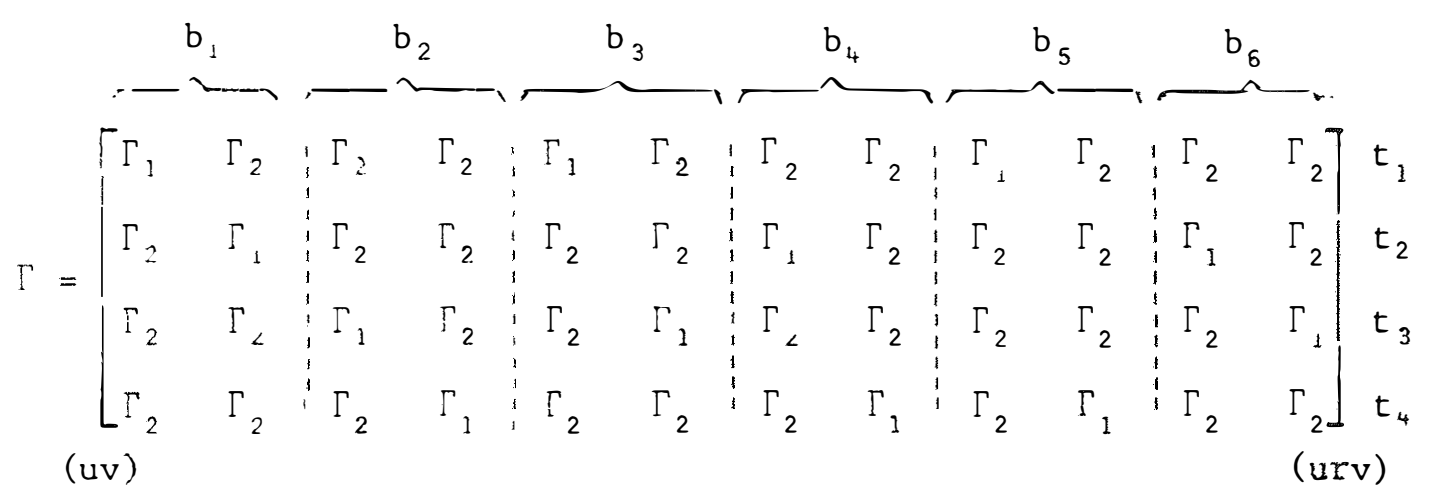

e, de modo geral, $\Gamma \bar{e}$ tal que suas submatrizes são iguais a

$$
\begin{aligned}
& \Gamma_{1} \text {, se o tratamento principal } \underline{i} \text { ocorre no bloco } \underline{j} \text {, } \\
& \Gamma_{2} \text {, caso contrário, }
\end{aligned}
$$

sendo essa generalização facilmente exequível, com base no esquema de blocos incompletos que se queira adotar.

Por sua vez, as submatrizes $\Gamma_{1}$ e $\Gamma_{2}$ são do tipo:

$$
\begin{aligned}
& \Gamma_{1}=I_{(\mathrm{u})}-\Omega-\psi+\Pi \\
& \Gamma_{1}=\frac{1}{u v}\left[\begin{array}{ccccc}
(\mathrm{u}-1)(\mathrm{v}-1) & -(\mathrm{v}-1) & -(\mathrm{v}-1) & \ldots & -(\mathrm{v}-1) \\
-(\mathrm{v}-1) & (\mathrm{u}-1)(\mathrm{v}-1) & -(\mathrm{v}-1) & \ldots & -(\mathrm{v}-1) \\
\ldots & \ldots & \ldots & \ldots & \ldots \\
-(\mathrm{v}-1) & -(\mathrm{v}-1) & -(\mathrm{v}-1) & & (\mathrm{u}-1)(\mathrm{v}-1)
\end{array}\right]
\end{aligned}
$$

(u) 
72 .

$\Gamma_{2}=-\Omega+\Pi$

$$
\therefore \quad \Gamma_{2}=\frac{1}{u v}\left[\begin{array}{ccccc}
-(u-1) & 1 & 1 & \ldots & 1 \\
1 & -(u-1) & 1 & \ldots & 1 \\
\ldots & \ldots & \ldots & \ldots & \ldots \\
1 & 1 & 1 & \ldots & -(u-1)
\end{array}\right]
$$

(u)

(u)

e, como de modo análogo a (4.5.1), analisando-se a expressão

$$
\mathrm{E}\left(\varepsilon \varepsilon^{\prime}\right) \Gamma^{\prime}
$$

obteve-se

$$
\begin{aligned}
E\left(\varepsilon \varepsilon^{\prime}\right) \Gamma^{\prime} & =(1-\rho) \sigma^{2} \Gamma^{\prime} \\
\therefore \quad \Gamma E\left(\varepsilon \varepsilon^{\prime}\right) \Gamma^{\prime} & =(1-\rho) \sigma^{2} \Gamma \Gamma^{\prime}
\end{aligned}
$$

assim, $(\alpha .35)$ ficou

$$
D(\hat{\delta})=\frac{(1-\rho) \sigma^{2}}{r^{2}} \cdot \Gamma \Gamma^{\prime}
$$

com $\Gamma \Gamma^{\prime}$ apresentando:

a) na diagonal principal

$$
\gamma_{\mathrm{ss}}=\mathrm{r} \Gamma_{1}^{2}+\mathrm{r}(\mathrm{v}-1) \Gamma_{2}^{2}=\mathrm{r}\left[\Gamma_{1}^{2}+(\mathrm{v}-1) \Gamma_{2}^{2}\right]
$$

b) fora da diagonal principal

$$
\gamma_{s^{\prime}}=r\left[2 \Gamma_{1} \Gamma_{2}+(v-2) \Gamma_{2}^{2}\right]
$$

Desenvolvendo-se, obteve-se 


$$
\gamma_{s s}=\frac{u r v}{u^{2} v^{2}}\left[\begin{array}{cccc}
(u-1)(v-1) & -(v-1) & \ldots & -(v-1) \\
-(v-1) & (u-1)(v-1) & \ldots & -(v-1) \\
\ldots & \ldots & \ldots & \ldots \\
-(v-1) & -(v-1) & \ldots & (u-1)(v-1) \\
(u) & & & (u)
\end{array}\right.
$$

e,

$$
\gamma_{s s^{\prime}}=\frac{u r v}{u^{2} v^{2}}\left[\begin{array}{cccc}
-(u-1) & 1 & \ldots & 1 \\
1 & -(u-1) & \ldots & 1 \\
\ldots & \ldots & \ldots & \ldots \\
1 & 1 & \ldots & -(u-1)
\end{array}\right]
$$

(u)

então, a expressão ( $\alpha .36)$ passou a ser
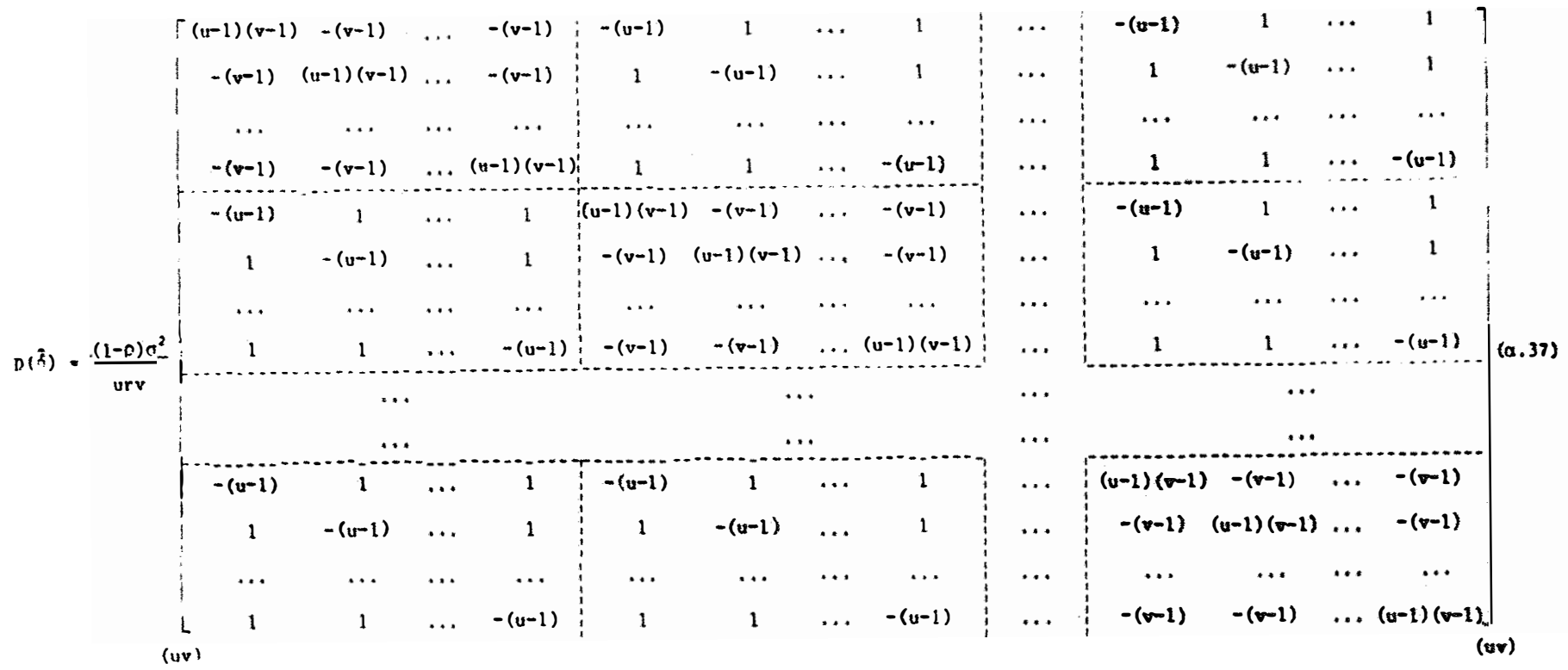
Assim, a variāncia para a diferença entre duas mëdias de efeitos estimados de tratamentos secundários dentro de um mesmo tratamento primārio ficou

$$
\begin{aligned}
\mathrm{v}\left(\hat{\delta}_{\text {is }}-\hat{\delta}_{\text {is }}{ }^{\prime}\right) & =2[(u-1)(v-1)+(v-1)] \frac{(1-\rho) \sigma^{2}}{u r v} \\
& =\frac{2(v-1)}{r v}(1-\rho) \sigma^{2} \\
\therefore \quad v\left(\hat{\delta}_{\text {is }}-\hat{\delta}_{\text {is }}{ }^{\prime}\right) & =\frac{2}{r}\left(1-\frac{1}{v}\right)(1-\rho) \sigma^{2}
\end{aligned}
$$

que è um resultado idêntico àquele apresentado por CHAKRABARTI(1962) para a mesma variância quando o ensaio com parcelas subdivididas édelineado em blocos (completos) casualizados, como pode ser observado em $(2.1 .3)$.

De modo anālogo à $(\alpha .27)$ e $(\alpha .33)$, uma estimativa pa ra essa variância foi obtida por

$$
\theta\left(\hat{\delta}_{\text {is }}-\hat{\delta}_{i s^{\prime}}\right)=\frac{2}{r}\left(1-\frac{1}{v}\right) \operatorname{QMRes}(b) \quad(\alpha .39)
$$

Tambēm, para a variância da diferença entre duas médias de efeitos estimados de tratamentos principais dentro de um mesmo tratamento secundārio, obteve-se

$$
\begin{aligned}
v\left(\hat{\delta}_{i s}-\hat{\delta}_{i \prime s}\right) & =2[(u-1)(v-1)+(u-1)] \frac{(1-p) \sigma^{2}}{u r v} \\
& =\frac{2(u-1)}{u r}(1-\rho) \sigma^{2}
\end{aligned}
$$




$$
\therefore \mathrm{V}\left(\bar{\delta}_{i s}-\widehat{\delta}_{i^{\prime} s}\right)=\frac{2}{r}\left(1-\frac{1}{u}\right)(1-0) \sigma^{2}
$$

resultado idêntico àquele apresentado por CHAKRABARTI (1962) e citado em (2.1.3) para essa variância, quando o experimento com parce las subdivididas é delineado em blocos (completos) casualizados. Naturalmente

$$
\bar{V}\left(\hat{\delta}_{i s}-\hat{\delta}_{i ' s}\right)=\frac{2}{r}\left(1-\frac{1}{u}\right) \operatorname{QMRes}(b)
$$

4.6 - Anāilise de Variāncia

\section{6 .1 - Introdução}

$$
\begin{aligned}
\text { De } Y=X \theta+ & \varepsilon, \text { obteve-se } \\
& \varepsilon^{\prime} \varepsilon=Y^{\prime} Y-\theta^{\prime} X^{\prime} Y
\end{aligned}
$$

e, tomando-se a estimativa para $\theta$, resultou a soma de quadrados do residuo:

$$
\text { SQRes }=Y^{\prime} Y-\hat{\theta}^{\prime} X^{\prime} Y
$$

então, SQRes = SQTotal - SQParâmetros, $\mathrm{SQReS}=\mathrm{SQT}-\mathrm{SQP}$.

$$
\text { Por outro lado, reportando-se a (، 3), onde } X^{\prime} Y=X^{\prime} X \hat{\theta} \text {, }
$$

$(x .42)$ ficou

$$
\text { SQRes }=Y^{\prime} Y-\hat{\theta}^{\prime} X^{\prime} X \hat{\theta},
$$

onde 


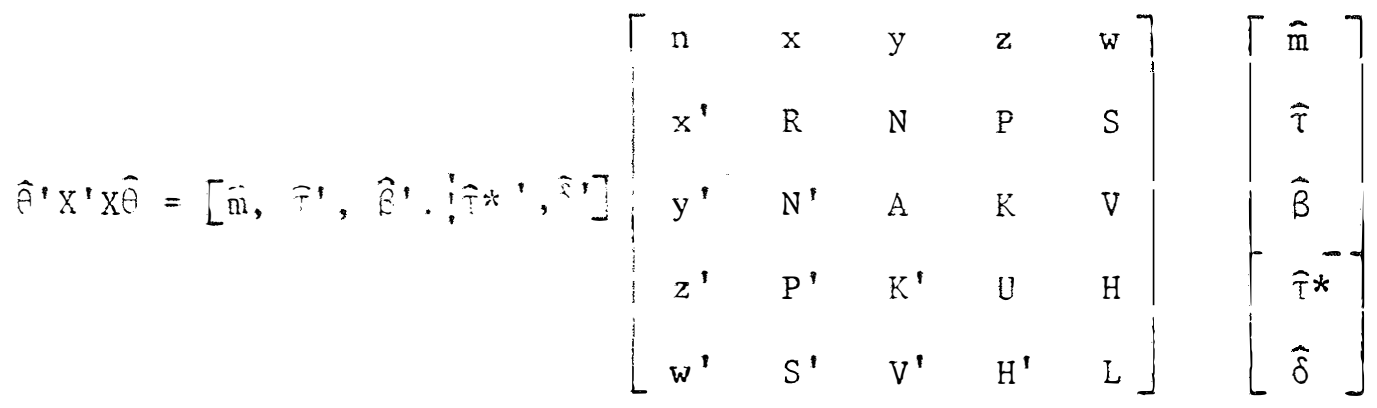

e a partição efetuada refere-se às duas "partes" encontradas nos es quemas usuais de análises de variância para ensaios com parcelas subdivididas em blocos casualizados.

Assim, para

$$
\begin{aligned}
& \vartheta_{1}=\left[\begin{array}{c}
\hat{\mathrm{m}} \\
\hat{\tau} \\
\hat{\beta}
\end{array}\right] \quad ; \quad \hat{\theta}_{2}=\left[\begin{array}{c}
\hat{\tau} * \\
\delta
\end{array} ; \quad\left(X^{\prime} X\right)_{a}=\left[\begin{array}{ccc}
n & x & y \\
X^{\prime} & R & N \\
y^{\prime} & N^{\prime} & A
\end{array}\right] ;\right. \\
& \left(X^{\prime} X\right)_{C}=\left[\begin{array}{cc}
z & w \\
K & V
\end{array}\right] ; \quad\left(X^{\prime} X\right)_{d}=\left[\begin{array}{lll}
z^{\prime} & P^{\prime} & K^{\prime} \\
W^{\prime} & S^{\prime} & V^{\prime}
\end{array}\right] ; \quad\left(X^{\prime} X\right)_{b}=\left[\begin{array}{ll}
U & H \\
H^{\prime} & L
\end{array}\right] ;
\end{aligned}
$$

resultou

$$
\begin{aligned}
\hat{\theta}^{\prime} X^{\prime} X \hat{\theta}= & {\left[\begin{array}{l:l:l}
\hat{\theta}_{1}^{\prime} & \hat{\theta}_{2}^{\prime}
\end{array}\right] \quad\left[\begin{array} { c : c } 
{ ( X ^ { \prime } X ) _ { a } } & { ( X ^ { \prime } X ) _ { c } } \\
{ \hdashline ( X ^ { \prime } X ) _ { d } } & { ( X ^ { \prime } X ) _ { b } }
\end{array} \left[\begin{array}{c}
\hat{\theta}_{1} \\
\left.\hdashline \hat{\theta}_{2}\right]
\end{array}\right.\right.} \\
= & \hat{\theta}_{1}^{\prime}\left(X^{\prime} X\right)_{a} \hat{\theta}_{1}+\hat{\theta}_{2}^{\prime}\left(X^{\prime} X\right)_{b} \hat{\theta}_{2}+\hat{\theta}_{2}^{\prime}\left(X^{\prime} X\right)_{d} \hat{\theta}_{1}+ \\
& +\hat{A}_{1}^{\prime}\left(X^{\prime} X\right)_{c} \hat{\theta}_{2}
\end{aligned}
$$


77.

onde

$$
\hat{\theta}^{\prime}\left(X^{\prime} X\right)_{\mathrm{d}} \hat{\theta}_{1}=\hat{\theta}_{1}^{\prime}\left(X^{\prime} X\right)_{\mathrm{c}} \hat{\theta}_{2}=\hat{\phi}
$$

pois .

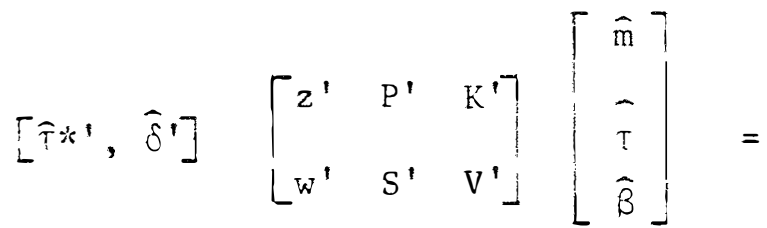

$$
\begin{aligned}
& =\left(\hat{\tau} *^{\prime} z^{\prime}+\hat{\delta}^{\prime} w^{\prime}\right) \hat{m}+\left(\hat{\tau} *^{\prime} P^{\prime}+\hat{\delta} S^{\prime}\right) \hat{\tau}+\left(\hat{\tau} *^{\prime} K^{\prime}+\hat{\delta} V^{\prime}\right) \hat{\beta}= \\
& =\hat{\tau} *^{\prime} z^{\prime} \hat{m}+\hat{\delta}^{\prime} w^{\prime} \hat{m}+\hat{\tau}^{*} P^{\prime} \hat{\tau}+\hat{\delta}^{\prime} S^{\prime} \hat{\tau}+\hat{\tau} *^{\prime} K^{\prime} \hat{B}+\hat{\delta}^{\prime} V^{\prime} \hat{B}
\end{aligned}
$$

$e$, desde que

$$
\begin{aligned}
& \widehat{T} *^{\prime} z^{\prime}=\operatorname{rv}: \frac{t_{S}^{\prime}=0}{S}=0 \\
& \bar{\delta}^{\prime} w^{\prime}=r \sum_{\text {is }}\left(\hat{t}^{\prime}\right)_{\text {is }}=0 \\
& \hat{\tau}^{*} P^{\prime}=\underset{(1)(v)}{\phi} \quad \Longrightarrow \quad \hat{\tau}^{*} P^{\prime} \hat{\tau}=0 \\
& \bar{\delta}^{\prime} S^{\prime}=\underset{(1)^{\phi}(v)}{\phi} \Longrightarrow \hat{\delta}^{\prime} S^{\prime} \bar{\tau}=0 \\
& \hat{\tau}^{*} K^{\prime}={ }_{(1)}^{\phi(a)} \Longrightarrow \hat{\tau}^{\prime} K^{\prime} \hat{B}=0 \\
& \hat{\delta}^{\prime} \mathrm{V}^{\prime}=\underset{(1)^{\phi}(\mathrm{a})}{\phi} \Longrightarrow \hat{\delta}^{\prime} \mathrm{V}^{\prime} \hat{p}=0
\end{aligned}
$$

então, $\hat{e}_{2}^{\prime}\left(x^{\prime} x\right)_{d} \hat{\theta}_{1}=0$, 
e, de modo análogo para a transposta

$$
\hat{\theta}_{1}^{i}\left(X^{i} X\right)_{c} \bar{\theta}_{2}^{i}=0
$$

desse modo, obteve-se

$$
\bar{\theta}^{\prime} X^{\prime} X \hat{\theta}=\hat{\theta}_{1}^{\prime}\left(X^{\prime} X\right)_{a} \vec{\theta}_{1}+\hat{\theta}_{2}^{\prime}\left(X^{\prime} X\right)_{b} \hat{\theta}_{2} \quad(\alpha .44)
$$

ou

$$
\mathrm{SQP}=\left[\hat{\mathrm{m}}, \hat{\tau}^{\prime}, \hat{R}^{\prime}\right]\left[\begin{array}{ccc}
\mathrm{n} & \mathrm{x} & \mathrm{y} \\
\mathrm{x}^{\prime} & \mathrm{R} & \mathrm{N} \\
\mathrm{y}^{\prime} & \mathrm{N}^{\prime} & A
\end{array}\right]\left[\begin{array}{c}
\hat{\mathrm{m}} \\
\hat{\tau} \\
\hat{\beta}
\end{array}\right]+\left[\hat{\tau}^{*}, \hat{\delta}^{\prime}\right]\left[\begin{array}{ll}
U & H \\
H^{\prime} & L
\end{array}\right]\left[\begin{array}{c}
\hat{\tau} * \\
\hat{\delta}
\end{array}\right]
$$

que, convencionou-se chamar por

$$
\begin{aligned}
\text { SQParāmetros } & =S Q P a r a ̂ m e t r o s(a)+S Q P a r a ̂ m e t r o s(b) \\
S Q P & =S Q P(a)+\operatorname{SQP}(b)
\end{aligned}
$$

assim, de $(\alpha .43)$, resultou

$$
\mathrm{SQRes}=\mathrm{SQT}-\mathrm{SQP}(\mathrm{a})-\mathrm{SQP}(\mathrm{b}) \cdot(\alpha .45)
$$

Mas,

$$
\text { SQTotal = SQTotal + SQParcelas - SQParcelas }
$$$$
\therefore \quad \mathrm{SQRes}=\{\mathrm{SQParc}-\mathrm{SQP}(\mathrm{a})\}+\{\mathrm{SQT}-\mathrm{SQParc}-\mathrm{SQP}(\mathrm{b})\}
$$

onde, SQParcelas foi definida por

$$
\operatorname{SQParc}=\frac{1}{u} Y^{\prime} \Phi Y-C_{0}
$$

com 


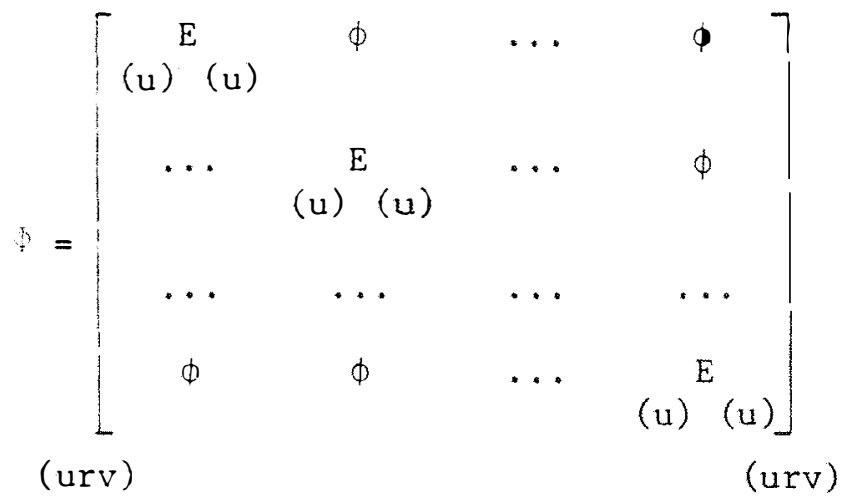

ou, como é usualmente encontrado na literatura

$$
\text { SQParc }=\frac{1}{u} \sum_{i, j} y_{i j}^{2} .-C_{0}
$$

Assim, obteve-se, de acordo com a terminologia tradi cional dos experimentos em parcelas subdivididas:

$$
\mathrm{SQReS}=\mathrm{SQReS}(\mathrm{a})+\mathrm{SQReS}(\mathrm{b}) .
$$

4.6.2 - Esperanças dos quadrados médios para os efeitos $f \underline{i}$ xos

4.6.2.1 - Soma de quadrados dos parãmetros

Retomando-se $(\alpha .42)$ obteve-se

$$
\mathrm{SQP}=\left[\hat{\mathrm{m}}, \hat{\tau}^{\prime}, \hat{\mathrm{B}}^{\prime}, \hat{\tau}^{*}, \hat{\delta}^{\prime}\right]\left[\begin{array}{c}
\mathrm{G} \\
\mathrm{T} \\
\mathrm{B} \\
\mathrm{T} * \\
\Delta
\end{array}\right]
$$


80.

$$
\therefore \quad \mathrm{SQP}=\hat{\mathrm{m}} \mathrm{G}+\hat{\tau}^{\prime} T+\hat{\beta} \cdot B+\hat{\tau} * T^{*} * \delta^{\prime} \Delta
$$

Entäo, por $(\alpha . *),(\alpha .15)$, obteve-se

$$
\begin{aligned}
& \mathrm{SQP}=\widehat{\mathrm{m}} \mathrm{G}+\widehat{T}^{\prime} \mathrm{T}+\mathrm{B}^{\prime} \mathrm{A}^{-1} \mathrm{~B}-\widehat{\tau}^{\prime} N A^{-1} B-\hat{\mathrm{m}} \mathrm{y} \mathrm{A}^{-1} \mathrm{~B}+\mathrm{T}^{\prime} \mathrm{U}^{-1} \mathrm{~T} *- \\
& \text { - } \hat{\mathrm{m}} z \mathrm{U}^{-1} \mathrm{~T}^{*}+\Delta^{2} \mathrm{~L}^{-1} \Delta-\mathrm{T}^{1} \mathrm{R}^{-1} \mathrm{SL}^{-1} \Delta-\mathrm{T}^{\prime} \mathrm{U}^{-2} \mathrm{HL} \mathrm{L}^{-1} \Delta+ \\
& +\hat{\mathrm{m}} w \mathrm{~L}^{-1} \Delta
\end{aligned}
$$

Agrupando-se os termos em $\hat{\imath}$ e usando-se o fato de que

$$
\hat{\mathrm{m}} \mathrm{y} A^{-1} B=\hat{\mathrm{m}} z U^{-1} \mathrm{~T}^{*}=\hat{\mathrm{m}} \mathrm{w} \mathrm{L}^{-1} \Delta=\hat{\mathrm{m}} \mathrm{G} \text {, }
$$

resultou

$$
\begin{aligned}
\mathrm{SQP}=\hat{\mathrm{m} G} & +\hat{\tau}^{\prime}\left(\mathrm{T}-\mathrm{N} A^{-1} \mathrm{~B}\right)+\mathrm{B}^{\prime} \mathrm{A}^{-1} \mathrm{~B}-\hat{\mathrm{m}} \mathrm{G}+\mathrm{T}^{*} \mathrm{U}^{-1} \mathrm{~T}- \\
& -\hat{\mathrm{m}} \mathrm{G}+\Delta^{\prime} \mathrm{L}^{-1} \Delta-\mathrm{T}^{\prime} \mathrm{R}^{-1} \mathrm{SL} \mathrm{L}^{-1} \Delta-\mathrm{T} *^{\prime} U^{-1} H L^{-1} \Delta+ \\
& +\hat{\mathrm{m}} \mathrm{S}
\end{aligned}
$$

onde:

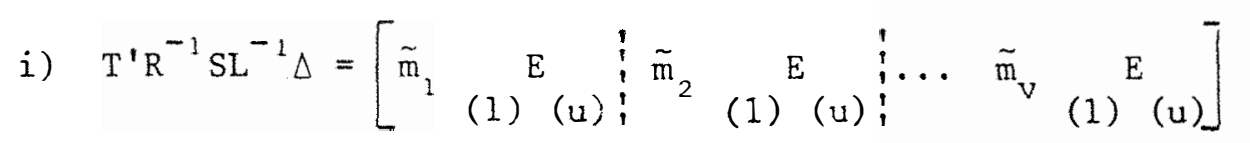


81.

$$
\begin{aligned}
& \mathrm{T}^{\prime} \mathrm{R}^{-1} \mathrm{SL}^{-}:=\tilde{\mathrm{m}}_{1} \mathrm{TT}_{1}^{\prime}+\tilde{\mathrm{m}}_{2} \mathrm{TT}_{2}^{\prime}+\ldots+\tilde{\mathrm{m}}_{\mathrm{v}} \mathrm{TT}_{\mathrm{v}}^{\prime} . \\
& =\left(\bar{m}+\tilde{t}_{1}\right) T_{1}+\left(\bar{m}+\tilde{t}_{2}\right) T_{2}+\ldots+ \\
& +\left(\hat{m}+\tilde{t}_{V}\right) T_{V} \\
& \therefore \quad \mathrm{T}^{\prime} \mathrm{R}^{-1} \mathrm{SL}^{-1} \Delta=\widehat{\mathrm{n}} \mathrm{G}+\tilde{\mathrm{T}}^{\prime} \mathrm{T} \text {. }
\end{aligned}
$$

ii) $\mathrm{T}^{\prime} \mathrm{U}^{-1} \mathrm{HL}{ }^{-1} \mathrm{~S}=$

$=\left[\hat{\mathrm{m}}_{1}^{\prime}, \hat{\mathrm{m}}_{2}^{\prime}, \ldots, \hat{\mathrm{m}}_{\mathrm{u}}^{\prime}: \ldots: \widehat{\mathrm{m}}_{1}^{\prime}, \hat{\mathrm{m}}_{2}^{\prime}, \ldots, \hat{\mathrm{m}}_{\mathrm{u}}^{1}\right]$

$$
\left[\begin{array}{c}
\mathrm{TT}_{i 2}^{\prime} \\
\mathrm{TT}_{\mathrm{i} 2}^{\prime} \\
\ldots \\
\mathrm{TT}_{1 \mathrm{u}}^{\prime} \\
\ldots \\
\ldots \\
\mathrm{TT}_{\mathrm{v}}^{\prime} \\
\mathrm{TT}_{\mathrm{v} 2}^{\prime} \\
\cdots \\
\mathrm{TT}_{\mathrm{vu}}^{\prime}
\end{array}\right]
$$

e, de modo análogo a (i), obteve-se

$$
\mathrm{T}^{*} \mathrm{U}^{-1} \mathrm{HL}{ }^{-1} \Delta=\hat{\mathrm{m}} \mathrm{G}+\hat{\tau} *^{\prime} \mathrm{T}^{*}
$$$$
E \text {, desde que } \widehat{\mathrm{m}} \mathrm{G}=\mathrm{C}_{\mathrm{O}}=\text { correção, de }(\alpha .48) \text {, resu1- }
$$

tou:

$$
\begin{aligned}
\mathrm{SQP}=\left\{\mathrm{C}_{0}\right\} & +\left\{\hat{\tau}^{\prime} \mathrm{Q}\right\}+\left\{\mathrm{B}^{\prime} \mathrm{A}^{-1} \mathrm{~B}-\mathrm{C}_{0}\right\}+\left\{\mathrm{T} *^{\prime} \mathrm{U}^{-1} \mathrm{~T}^{*}-\mathrm{C}_{0}\right\}+ \\
& +\left\{\Delta^{\prime} \mathrm{L}^{-1} \Lambda-\mathrm{C}_{0}-\tilde{\tau}^{\prime} \mathrm{T}-\hat{\tau}^{\prime} \mathrm{T}^{*}\right\}
\end{aligned}
$$


82.

onde, $\hat{\tau}^{\prime} Q=$ soma de quadrados devida aos efeitos dos tratamentos principais, ajustada para efeitos de blocos:

$$
\hat{\tau}^{\prime} Q=\operatorname{SQt}(\mathrm{aj} \cdot)
$$

$B^{\prime} A^{-1} B-C_{0}=$ soma de quadrados devida aos efeitos de blocos, obtida de modo usual:

$$
\begin{aligned}
& B^{\prime} A^{-1} B-C_{0}=\frac{1}{u k} \sum_{j=1}^{a} B_{j}^{2}-C_{0}=S Q b ; \quad(\alpha .49 .2) \\
& \mathrm{T} *^{\prime} \mathrm{U}^{-1} \mathrm{~T} *-\mathrm{C}_{0}=\text { soma de quadrados devida aos efeitos dos tratamen- } \\
& \text { tos secundários, obtida de modo usual: } \\
& \mathrm{T}^{*} \mathrm{U}^{-1} \mathrm{~T}^{*}-\mathrm{C}_{\mathrm{O}}=\frac{1}{\mathrm{rV}} \sum_{\mathrm{s}=1}^{\mathrm{u}} \mathrm{T}_{\mathrm{S}}^{2}-\mathrm{C}_{\mathrm{O}}=\mathrm{SQ} \mathrm{t}^{\prime} ; \quad(\alpha .49 .3) \\
& \Delta^{\prime} L^{-1} \Delta-C_{0}-\tilde{\tau}^{\prime} T-\tilde{\tau}^{\prime} T *=\text { soma de quadrados devida aos efeitos da } \\
& \text { interação, } \mathrm{SQ \delta} \text {, onde } \\
& \Delta^{\prime} \mathrm{L}^{-1} \Delta=\frac{1}{\mathrm{r}} \sum_{\mathrm{i}, \mathrm{s}}\left(\mathrm{TT}^{\prime}\right)_{\text {is }}^{2} \\
& \tilde{\tau}^{\prime} T=T^{\prime} R^{-1} T-C_{0}=\frac{1}{u r} \sum_{i=1}^{v} T_{j}^{2}-C_{0}=\text { soma de quadrados devida aos } \\
& \text { efeitos dos tratamentos princi } \\
& \text { pais, obtida de modo usual, sem } \\
& \text { a correção para efeitos de blo } \\
& \cos \\
& \widehat{\tau} *^{\prime} \mathrm{T}^{*}=\mathrm{T}^{*} \mathrm{U}^{-1} \mathrm{~T} *-\mathrm{C}_{\mathrm{O}}=\mathrm{SQ} \mathrm{t}^{\prime} \\
& \therefore \quad \operatorname{SQ} \delta=\frac{1}{r} \sum_{i, S}\left(\mathrm{IT}^{\prime}\right)_{i s}^{2}-\mathrm{C}_{\mathrm{O}}-\mathrm{SQt} \text { (usual) } \\
& -S Q t^{\prime} \\
& (\alpha .49 .4)
\end{aligned}
$$


83.

Tendo-se em conta que na anālise de variância, o prí meiro termo da SQP (em $\alpha .49$ ) é usado na correção da SQTotal, obteve-se

$$
\mathrm{SQP}=\mathrm{SQt}(\mathrm{aj} .)+\mathrm{SQb}(\text { usual })+\mathrm{SQt}^{\prime}(\text { usual })+\mathrm{SQ \delta} \quad(\alpha .50)
$$

então

$$
\begin{aligned}
& E[S Q P]=E[S Q t(a j \cdot)]+E[S Q b]+E\left[S Q t^{\prime}\right]+E[S Q \delta] \\
& \text { 4.6.2.2.1 - Mēdia geral } \\
& \operatorname{SQ} \hat{\mathrm{m}}=\hat{\mathrm{m}} \mathrm{G}=\mathrm{C}_{\mathrm{O}}=\frac{\mathrm{G}^{2}}{\mathrm{urv}} \\
& \therefore E\left[S \hat{m}^{-}\right]=\frac{1}{\operatorname{urv}} E^{-}\left[G^{2}\right]=\frac{1}{\operatorname{urv}}\left[\left(X_{1}^{\prime} Y\right)^{\prime}\left(X_{1}^{\prime} Y\right)\right] \\
& =\frac{1}{\text { urv }} E\left[\left(\theta^{\prime} X^{\prime}+\varepsilon^{\prime}\right) X_{1} X_{1}^{\prime}(X \theta+\varepsilon)\right] \\
& =\frac{1}{n} E\left[\theta^{\prime} X^{\prime} X_{1} X_{1}^{\prime} X \theta+\theta^{\prime} X^{\prime} X_{1} X_{1}^{\prime} \varepsilon+\varepsilon^{\prime} X_{1} X_{1}^{\prime} X \theta+\varepsilon^{\prime} X_{1} X_{1}^{\prime} \varepsilon\right] \\
& \therefore E[S Q \hat{m}]=\frac{1}{n}\left\{\theta^{\prime} X^{\prime} X_{1} X_{1}^{\prime} X \theta+E\left[\varepsilon^{\prime} X_{1} X_{1}^{\prime} \varepsilon\right]\right\}
\end{aligned}
$$

onde, 
84.

i)

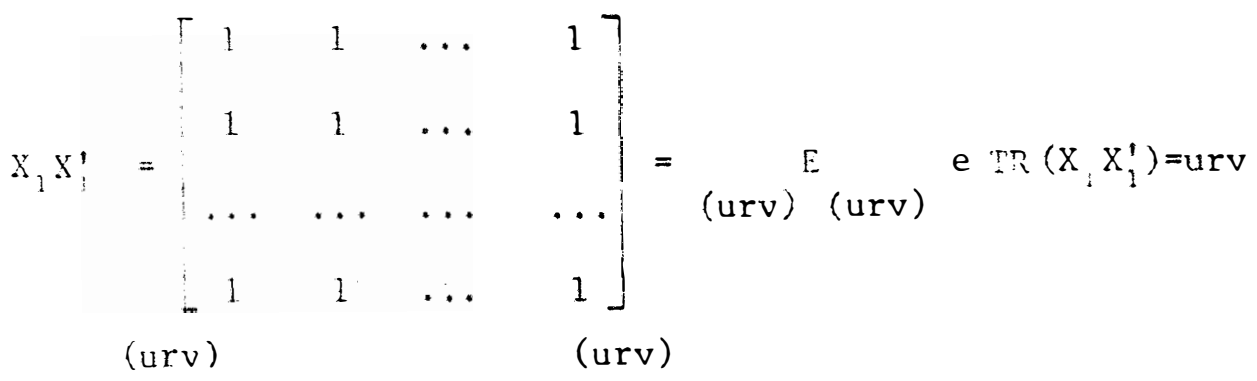

Considerando-se que para blocos incompletos balance $\underline{a}$ dos $a k=r v$, então,

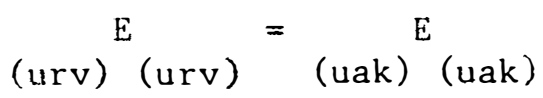

e partindo-se a matriz (uak) (uak) em a submatrizes quadradas de dimensão uk, (a blocos, cada qual com $\underline{\mathrm{k}}$ parcelas de $\underline{\mathrm{u}}$ subparcelas), obteve-se, sem perda de generalidade, para a $1^{a}$. submatriz e conside rando-se o exemplo inicial:

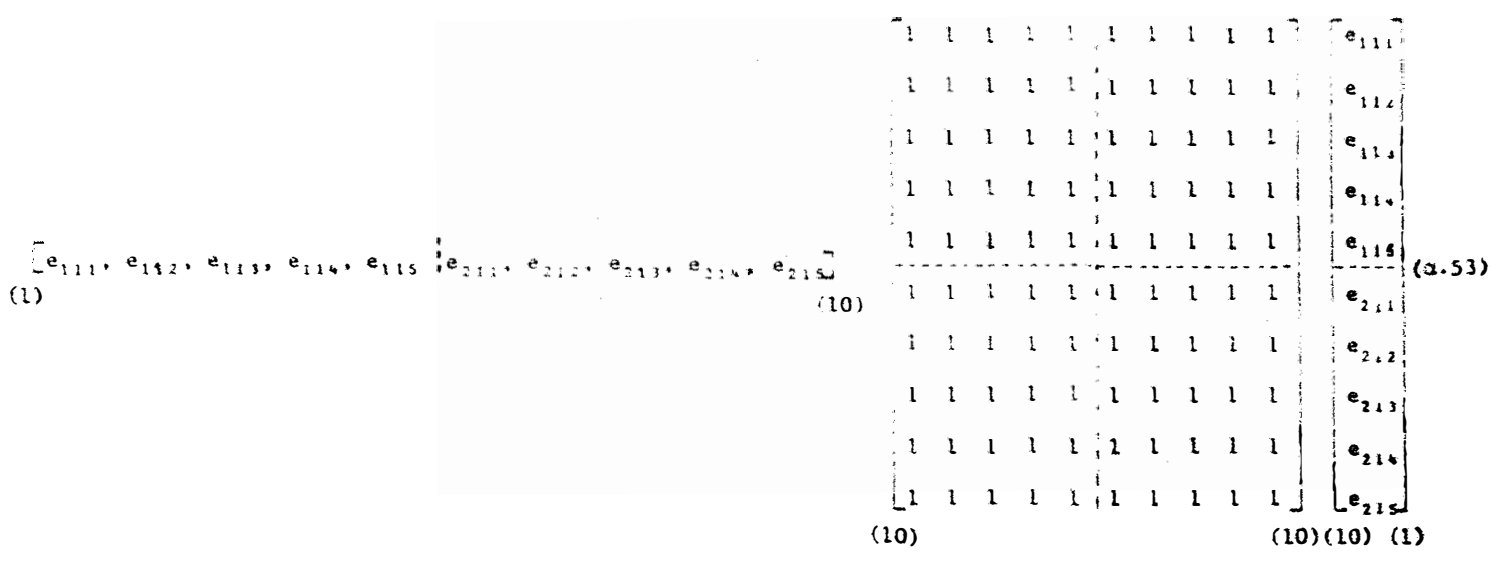


Como é fäcil ver, à partição proposta para $X_{1} X_{1}^{\prime}$ corresponderam partições anälogas em $\varepsilon$ e $\varepsilon^{\prime}$.

Caracterizando-se, então, $(\alpha .53)$ por

$$
\left[\begin{array}{l:l:l}
\varepsilon_{1}^{1} & \varepsilon_{2}^{1}
\end{array}\right]\left[\begin{array}{c:c}
\left(X_{1} X_{1}^{\prime}\right)_{a} & \left(X_{1} X_{1}^{\prime}\right)_{b} \\
\hdashline\left(X_{1} X_{1}^{\prime}\right)_{c} & \left(X_{1} X_{1}^{\prime}\right)_{d}
\end{array}\right]\left[\begin{array}{c}
\varepsilon_{1} \\
\hdashline \varepsilon_{2}
\end{array}\right]
$$

e efetuando-se o produto, obteve-se

$$
\begin{aligned}
& \left\{\varepsilon_{1}^{\prime}\left(X_{1} X_{1}^{\prime}\right)_{a}+\varepsilon_{2}^{\prime}\left(X_{1} X^{\prime}\right)_{c}\right\} \varepsilon_{1}+\left\{\varepsilon_{1}^{\prime}\left(X_{1} X_{1}^{\prime}\right)_{b}+\varepsilon_{2}^{\prime}\left(X_{1} X_{1}^{\prime}\right)_{d}\right\} \varepsilon_{2}= \\
= & \varepsilon_{1}^{\prime}\left(X_{1} X_{1}^{\prime}\right)_{a} \varepsilon_{1}+\varepsilon_{2}^{\prime}\left(X_{1} X_{1}^{\prime}\right)_{c} \varepsilon_{1}+\varepsilon_{1}^{\prime}\left(X_{1} X_{1}^{\prime}\right)_{b} \varepsilon_{2}+\varepsilon_{2}^{\prime}\left(X_{1} X_{1}^{\prime}\right)_{d} \varepsilon_{2}
\end{aligned}
$$

onde, $\operatorname{TR}\left(\mathrm{X}_{1} \mathrm{X}_{1}^{\prime}\right)_{\mathrm{a}}=\operatorname{TR}\left(\mathrm{X}_{1} \mathrm{X}_{1}^{\prime}\right)_{\mathrm{b}}=\operatorname{TR}\left(\mathrm{X}_{1} \mathrm{X}_{1}^{\prime}\right)_{\mathrm{c}}=\operatorname{TR}\left(\mathrm{X}_{1} \mathrm{X}_{1}^{\prime}\right)_{\mathrm{d}}=\mathrm{u}=5$.

Por outro lado, considerando-se que uma matriz qua$\operatorname{drada}(u)^{E}(u)$ tem $\operatorname{TR}\left[(u)^{E}(u)\right]=u$ elementos na diagonal principal e $u^{2}-\operatorname{TR}[(u) E(u)]=u(u-1)$ elementos fora da diagonal principal, obteve-se, para $(\alpha .54)$, em termos de esperanças, e usando-se o fato de que:

$$
\begin{aligned}
& \operatorname{Cov}\left(y_{i j s}, y_{i} j^{\prime} s^{\prime}\right)=\left\{\begin{array}{l}
\sigma^{2}, \text { se } i=i^{\prime}, j=j^{\prime} \text { e } s=s^{\prime} \\
\rho \sigma^{2}, \text { se } i=i^{\prime}, j=j^{\prime} \text { e } s \neq s^{\prime} \quad \text { (por 4.2) } \\
0, \text { em outros casos }
\end{array}\right.
\end{aligned}
$$

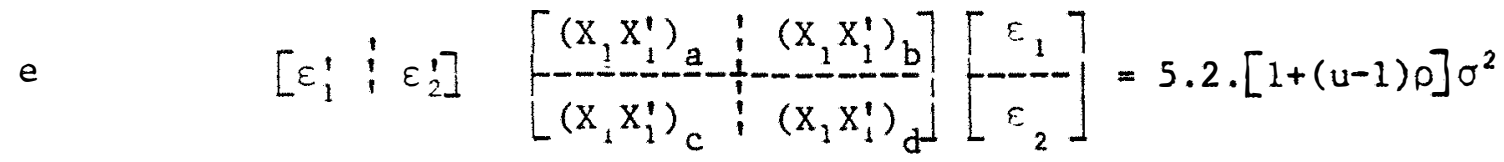

e genericamente, para um bloco qualquer com $\underline{k}$ parcelas de $\underline{u}$ subpar celas, ficou 


$$
u k[1+(u-1) \rho] \sigma^{2}
$$

e, desse modo, para os a blocos de um ensaio qualquer, obteve-se para 029 termo de $(x .52)$ :

$$
\begin{aligned}
& E\left[\varepsilon^{\prime} x_{1} x_{1}^{\prime} \varepsilon\right]=u a k[1+(u-1) \rho] \sigma^{2} \\
\text { Mas, uak }=u r v=n, & \text { urv }=n \\
\therefore \quad & E\left[\varepsilon^{\prime} x_{1} x_{1}^{\prime} \varepsilon\right]=n[1+(u-1) p] \sigma^{2}
\end{aligned}
$$

ii) No desenvolvimento do termo $\theta^{\prime} x^{\prime} x_{1} x_{1}^{\prime} x \theta$ de $(\alpha .52)$, tomou-se

$$
\begin{aligned}
& x_{1}^{\prime} x^{\prime}=x_{1}^{\prime}\left[x_{1}: x_{2}: x_{3}: x_{4}: x_{3}\right]=[n, x, y, z, w] \\
& \therefore \quad x^{\prime} x_{1} x_{1}^{\prime} x=\left[\begin{array}{ccccc}
n^{2} & n x & n y & n z & n w \\
x^{\prime} n & x^{\prime} x & x^{\prime} y & x^{\prime} z & x^{\prime} w \\
y^{\prime} n & y^{\prime} x & y^{\prime} y & y^{\prime} z & y^{\prime} w \\
z^{\prime} n & z^{\prime} x & z^{\prime} y & z^{\prime} z & z^{\prime} w \\
w^{\prime} n & w^{\prime} x & w^{\prime} y & w^{\prime} z & w^{\prime} w
\end{array}\right]
\end{aligned}
$$

donde,

$$
\begin{aligned}
\theta^{\prime} X^{\prime} X_{1} X_{1}^{\prime} X \theta= & \left(m n^{2}+\tau^{\prime} x^{\prime} n+\beta^{\prime} y^{\prime} n+\tau^{\prime} z^{\prime} n+\delta^{\prime} w^{\prime} n\right) m+ \\
& +\left(m n x+\tau^{\prime} x^{\prime} x+\beta^{\prime} y^{\prime} x+\tau^{\prime} z^{\prime} x+\delta^{\prime} w^{\prime} x\right) \tau+ \\
& +\left(m n y+\tau^{\prime} x^{\prime} y+\beta^{\prime} y^{\prime} y+\tau^{\prime} z^{\prime} y+\delta^{\prime} w^{\prime} y\right) \beta+ \\
& +\left(m n z+\tau^{\prime} x^{\prime} z+\beta^{\prime} y^{\prime} z+\tau^{\prime} z^{\prime} z+\delta^{\prime} w^{\prime} z\right) \tau *+ \\
& +\left(m n w+\tau^{\prime} x^{\prime} w+\beta^{\prime} y^{\prime} w+\tau^{\prime} z^{\prime} w+\delta^{\prime} w^{\prime} w\right) \delta
\end{aligned}
$$


87.

que, ao efetuar-se os produtos e aplicar-se as restrições impostas em $(4.2)$, resultou:

$$
\theta^{\prime} x^{\prime} x_{1} x_{1}^{\prime} x=n^{2} m^{2}
$$

Substituindo-se, então, $(\alpha .55)$ e $(\alpha .56)$ em $(\alpha .52)$, re

sultou:

$$
\begin{aligned}
& E[S Q \hat{m}]=\frac{1}{n}\left\{n[1+(u-1) \rho] \sigma^{2}+n^{2} m^{2}\right\} \\
\therefore \quad & E[S Q \hat{m}]=E[C \sigma]=[1+(u-1) \rho] \sigma^{2}+n m^{2} \quad(\alpha .57)
\end{aligned}
$$

\subsubsection{2 - Tratamentos principais}

$$
\operatorname{SQt}\left(a_{j} \cdot\right)=\hat{\tau}^{\prime} Q=Q^{\prime} M^{-1} Q
$$

Para minimizar os cälculos devidos à substituição de $\mathrm{Q}=\mathrm{T}-\mathrm{NA}{ }^{-1} \mathrm{~B}(\alpha .6 .1)$, adotou-se o uso da matriz que em CHARRABARTI (1962), PIMENTEL GOMES (1967) e DINIZ (1980), recebeu o nome de matriz $W$ e que neste estudo foi definida por

$$
\Lambda=\left[(v)^{\phi}(1) ; I_{(v)} ; \quad \underset{(v)}{\left(-\mathrm{NA}^{-1}\right)}{ }_{(\mathrm{a})}^{;}(\mathrm{v})^{\phi}(\mathrm{u}) ;(\mathrm{v}){ }^{\phi}(\mathrm{uv})\right] \text {. }
$$

Usando-se a propriedade demonstrada por PIMENTEL GOMES (1967) de que $Q=\Lambda X^{\prime} Y$, obteve-se:

$$
\begin{aligned}
\operatorname{sit}(\operatorname{aj} .) & =Y^{\prime} X \Lambda^{\prime} M^{-1} \Lambda X^{\prime} Y \\
& =\left(\theta^{\prime} X^{\prime}+\varepsilon^{\prime}\right) X \Lambda^{\prime} M^{-1} \Lambda X^{\prime}(X \theta+\varepsilon)
\end{aligned}
$$

donde, 


$$
E[\operatorname{SQt}(a j .)]=\theta^{\prime} X^{\prime} X A^{\prime} M^{-1} A X^{\prime} X \theta+E\left[\varepsilon^{\prime} X \Lambda^{\prime} M^{-1} \Lambda X^{\prime} \varepsilon\right] .
$$

Em sequência, para outros resultados obtidos por PIMENTEL GOMES (1967)

$$
\triangle X^{\prime} X \theta=C \tau=Q \quad \text { e } \quad \Lambda X^{\prime} X \Lambda^{\prime}=C \text {, }
$$

resultou

$$
E[\operatorname{SQt}(a j \cdot)]=\tau^{\prime} C^{\prime} M^{-1} C \tau+E\left[\varepsilon^{\prime} X \Lambda^{\prime} M^{-1} A X^{\prime} \varepsilon\right]
$$

e, desde que $C^{\prime} M^{-1} C=C M^{-1} C=C, e$, ainda, $C \tau=Q$,

$$
E[\operatorname{sQt}(a j .)]=\tau^{\prime} Q+E\left[\varepsilon^{\prime} X \Lambda^{\prime} M^{-1} \Lambda X^{\prime} \varepsilon\right] . \quad(\alpha .58)
$$

Para o desenvolvimento de $E\left[\varepsilon^{\prime} X \Lambda^{\prime} M^{-1} \Lambda X^{\prime} \varepsilon\right]$ definiu-se $\Pi=X \Lambda^{\prime} M^{-1} \Lambda X^{\prime}$, obtendo-se $E\left[\varepsilon^{\prime} \Pi \varepsilon\right]$ e com raciocínio análogo ao desenvolvido para demonstrar-se $(\alpha .55)$, provou-se que

$$
\begin{gathered}
E\left[\varepsilon^{\prime} \Pi \varepsilon\right]=\operatorname{TR}(\Pi)[1+(u-1) \rho] \sigma^{2} \\
\text { Partindo-se, então, a matriz } \Pi \text { em } \\
\Pi_{1}=X A^{\prime} \text { e } \Pi_{2}=M^{-1} A X^{\prime},
\end{gathered}
$$

e usando-se a propriedade de que se existem os produtos $\pi_{1} \Pi_{2}$ e $\pi_{2} \Pi_{1}$ então $\operatorname{TR}\left(\Pi_{1} \Pi_{2}\right)=\operatorname{TR}\left(\Pi_{2} \Pi_{1}\right)$ resultou

$$
\operatorname{TR}(I)=\operatorname{TR}\left(M^{-1} \Lambda X^{\prime} X \Lambda^{\prime}\right)=\operatorname{TR}\left(M^{-1} C\right) \text {, }
$$

onde $\mathrm{M}^{-1} \mathrm{C}$ é idempotente.

Então

$$
\ell\left(M^{-1} C\right)=T R\left(M^{-1} C\right)
$$


Tendo-se em conta que $C$ é singular de característica $\ell=v-1$, então, como $M^{-1} \bar{e}$ diagonal e, por consequência, não singular, o produto $\mathrm{M}^{-1} \mathrm{C}$ preserva a característica de $\mathrm{C}$.

$$
\therefore \operatorname{TR}\left(M^{-1} C\right)=\ell(C)=v-1
$$

obtendo-se, para ( $\alpha .59)$

$$
E\left[\varepsilon^{\prime} \Pi \varepsilon\right]=(\mathrm{v}-1)[1+(\mathrm{u}-1) 0] \sigma^{2} \quad(\alpha .60)
$$

Assim, de $(\alpha .58)$ e $(\alpha .60)$, resultou

$E[\operatorname{SQt}(\mathrm{aj} \cdot)]=(\mathrm{v}-1)[1+(\mathrm{u}-1) \rho] \sigma^{2}+\tau^{\prime} \mathrm{Q} \quad(\alpha .61)$

Da característica de $C$, obteve-se o nümero de graus de liberdade para tratamentos principais.

$$
\begin{aligned}
& \text { Desse modo, } \\
& E[\operatorname{QMt}(\operatorname{aj} .)]=[1+(u-1) 0] \sigma^{2}+\frac{\tau^{\prime} Q}{v-1} \\
& 4.6 .2 .2 .3-810 \cos \\
& \mathrm{SQb}=\mathrm{B}^{\prime} \mathrm{A}^{-1} \mathrm{~B}-\mathrm{C}_{\mathrm{O}} \\
& =\left(X_{3}^{\prime} Y\right)^{\prime} A^{-1}\left(X_{3}^{\prime} Y\right)-C_{0} \\
& =\left(\theta^{\prime} X^{\prime}+\varepsilon^{\prime}\right) X_{3} A^{-1} X_{3}^{\prime}(X \theta+\varepsilon)-C_{0} \\
& \therefore E[S Q b]=\theta^{\prime} X^{\prime} X_{3} A^{-1} X_{3}^{\prime} X \theta+E\left[\varepsilon^{\prime} X_{3} A^{-1} X_{3}^{\prime} \varepsilon\right]-E[C]
\end{aligned}
$$




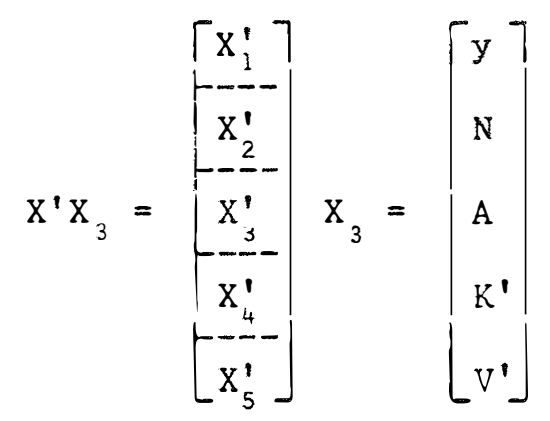

$$
\begin{aligned}
& \therefore X^{\prime} X_{3} A^{-1} X_{3}^{\prime} X=\left[\begin{array}{ccccc}
y A^{-1} y^{\prime} & y^{-1} N^{\prime} & y & y^{-1} K & y A^{-1} V \\
N A^{-1} y^{\prime} & N^{-1} N^{\prime} & N & N^{-1} K & N A^{-1} V \\
y^{\prime} & N^{\prime} & A & K & V \\
K^{\prime} A^{-1} y^{\prime} & K^{\prime} A^{-1} N^{\prime} & K^{\prime} & K^{8} A^{-1} K & K^{\prime} A^{-1} V \\
V^{\prime} A^{-1} y^{\prime} & V^{\prime} A^{-1} N^{\prime} & V^{\prime} & V^{\prime} A^{-1} K & V^{\prime} A^{-1} V
\end{array}\right]
\end{aligned}
$$

Então, de modo anālogo ao usado na obtenção de $(\alpha .56)$, efetuando-se o produto e aplicando-se as restrições, obteve-se:

$$
\theta^{\prime} X^{\prime} X_{3} A^{-1} X_{3}^{\prime} X \theta=n m^{2}+\tau^{\prime} N A^{-1} N^{\prime} \tau+2 B^{\prime} N^{\prime} \tau+B^{\prime} A B
$$

e como, de $(\alpha .6 .1) \mathrm{R}-\mathrm{C}=\mathrm{NA}^{-1} \mathrm{~N}^{\prime},(\alpha .63)$ resultou

$$
\begin{aligned}
E[S Q b]=n m^{2} & +\tau^{\prime} R \tau-\tau^{\prime} Q+2 B^{\prime} N^{\prime} \tau+B^{\prime} A B+ \\
& +E\left[\varepsilon^{\prime} X_{3} A^{-1} X_{3}^{\prime} \varepsilon\right]-E[C]
\end{aligned}
$$

Por sua vez, como em $(\alpha .59)$, fazendo-se

$$
\pi_{3}=x_{3} A^{-1} X_{3}^{1}
$$

obteve-se

$$
E\left[\varepsilon^{\prime} \Pi_{3} \varepsilon\right]=\operatorname{TR}\left(\Pi_{3}\right)[1+(u-1) \rho] \sigma^{2}
$$

e, para $\Pi_{4}=x_{3}$ e $\Pi_{5}=A^{-1} X_{3}^{\prime}$, 


$$
\operatorname{TR}\left(\Pi_{3}\right)=\operatorname{TR}\left(\Pi_{5} \Pi_{4}\right)=\operatorname{TR}\left(I_{a}\right)=a
$$

Assim, $(\alpha .64)$ ficou:

$$
\begin{aligned}
& E[S Q b]=n m^{2}+\tau^{\prime} R \tau-\tau^{\prime} Q+2 B^{\prime} N^{\prime} \tau+B^{\prime} A B+a[1+(u-1) D] \sigma^{2}- \\
& -\mathrm{n} \mathrm{m}^{2}-[1+(\mathrm{u}-1) \rho] \sigma^{2} \\
& \therefore E[S Q b]=(a-1)[1+(u-1) \rho] \sigma^{2}+\tau^{\prime} R \tau-\tau^{\prime} Q+2 B^{\prime} N^{\prime} \tau+ \\
& +B^{\prime} A \beta
\end{aligned}
$$

4.6.2.2.4 - Tratamentos secundārios

$$
\begin{aligned}
S Q t^{\prime} & =T^{*} U^{-1} T^{*}-C_{0} \\
& =Y^{\prime} X_{4} U^{-1} X_{4}^{\prime} Y-C_{0} \\
\therefore \quad S Q t^{\prime} & =\left(\theta^{\prime} X^{\prime}+\varepsilon^{\prime}\right) X_{4} U^{-1} X_{4}^{\prime}(X \theta+\varepsilon)-C_{0}
\end{aligned}
$$

Então,

$$
E\left[S Q t^{\prime}\right]=\theta^{\prime} X^{\prime} X_{4} U^{-1} X_{4}^{\prime} X \theta+E\left[\varepsilon^{\prime} X_{4} U^{-1} X_{4}^{\prime} \varepsilon\right]-E\left[C_{\sigma}\right] \quad(\alpha .67)
$$

onde,

$$
X^{\prime} X_{4}=\left[\begin{array}{c}
z \\
P \\
K \\
U \\
H^{\prime}
\end{array}\right]
$$

então, 
92.

$$
X^{\prime} X_{4} U^{-1} X_{4}^{\prime} X=\left[\begin{array}{ccccc}
n & x & y & z & W^{-1} \\
x^{\prime} & P U^{-1} P^{\prime} & P^{-1} K^{\prime} & P & P U^{-1} H \\
y^{\prime} & K U^{-1} P^{\prime} & K U^{-1} K^{\prime} & K & K U^{-1} H \\
z^{\prime} & P^{\prime} & K^{\prime} & U^{\prime} & H \\
W^{\prime} & H^{\prime} U^{-1} P^{\prime} & H^{\prime} U^{-1} K^{\prime} & H^{\prime} & H^{\prime} U^{-1} H
\end{array}\right]
$$

e, de modo anālogo a $(\alpha .56)$, efetuando-se o produto e aplicando-se as restrições, foi obtido

$$
\theta^{\prime} X^{\prime} X_{4} U^{-1} X_{4}^{\prime} X \theta=n m^{2}+\tau *^{\prime} U \tau^{*}
$$

Assim, $(\alpha .67)$ passou a ser

$$
\begin{aligned}
E\left[S Q t^{\prime}\right] & =n m^{2}+\tau^{*} U \tau^{*}+E\left[\varepsilon^{\prime} X_{4} U^{-1} X_{4}^{\prime} \varepsilon\right]-E\left[C_{0} \quad(\alpha .68)\right. \\
& \text { Por sua vez, no desenvolvimento do termo } E\left[\varepsilon^{\prime} X_{4} U^{-1} X_{4}^{\prime} \varepsilon\right],
\end{aligned}
$$
adotou-se $\Pi_{6}=x_{4} U^{-1} x_{4}^{\prime}, \Pi_{7}=x_{4}$ e $\Pi_{8}=U^{-1} x_{4}^{\prime}$,

$$
\therefore \operatorname{TR}\left(X_{4} U^{-1} X_{4}^{\prime}\right)=\operatorname{TR}\left(U^{-1} X_{4}^{\prime} X_{4}\right)=\operatorname{TR}\left(U^{-1}: 1\right)=\operatorname{TR}[I(u)]=u
$$

No entanto, se para a correção $(\alpha .55)$, tratamentos

principais $(\alpha .60)$ e para blocos $(\alpha .65)$ foram obtidas

$$
E\left[\varepsilon^{\prime} \Pi * \varepsilon\right]=[1+(u-1) \rho] \sigma^{2}+f(P),
$$

onde $\Pi *$ é uma das matrizes ali definidas, e $f(P) \vec{e}$ uma função dos pạ râmetros envolvidos, aqui o resultado obtido para o termo que envol ve a variância foi diferente.

Este fato foi devido à estrutura da matriz $x_{4}$, que $\vec{e}$ composta de submatrizes I(u) 


$$
\begin{aligned}
& x_{4}=\left[\begin{array}{c}
I(u) \\
I(u) \\
\ldots \\
I_{(u)}
\end{array}\right] \\
& \text { (urv) (u) }
\end{aligned}
$$

então, do produto $x_{4} X_{4}^{\prime}$ resultou uma matriz do tipo

$$
\begin{aligned}
& X_{4} X_{4}^{\prime}=\left[\begin{array}{cccc}
I_{(u)} & I_{(u)} & \cdots & I_{(u)} \\
I_{(u)} & I_{(u)} & \ldots & I_{(u)} \\
\ldots & \ldots & \cdots & \ldots \\
I_{(u)} & I_{(u)} & \cdots & I_{(u)}
\end{array}\right] \\
& \text { (urv) (urv) }
\end{aligned}
$$

onde, no produto $\frac{1}{\mathrm{rv}} \varepsilon^{\prime} \mathrm{X}_{4} \mathrm{X}_{4}^{\prime} \varepsilon$ para a primeira submatriz $I_{(\mathrm{u})}$, obteve-se (1. parcela):

$$
\begin{gathered}
\frac{1}{\operatorname{rv}}\left[e_{111}, e_{112}, \ldots, e_{11 u}\right]\left[\begin{array}{ccccc}
1 & 0 & 0 & \ldots & 0 \\
0 & 1 & 0 & \ldots & 0 \\
\ldots & \ldots & \ldots & \ldots & \ldots \\
0 & 0 & 0 & \ldots & 1
\end{array}\right]\left[\begin{array}{l}
e_{111} \\
e_{112} \\
\ldots \\
e_{11 u}
\end{array}\right]= \\
=\frac{1}{r v}\left(e_{111}^{2}+e_{112}^{2}+\ldots+e_{11 u}^{2}\right)
\end{gathered}
$$

Assim, em termos de esperança, ficou

$$
\frac{1}{\mathrm{rv}} \operatorname{TR}\left[\mathrm{I}_{(\mathrm{u})}\right] \sigma^{2}=\frac{\mathrm{u}}{\mathrm{rv}} \sigma^{2} \text {, }
$$


94.

então, para as rv submatrizes, is to é, para todas as parcelas do en saio

$$
E\left[\varepsilon^{\prime} X_{4} U^{-1} X_{4}^{1}\right]=u \sigma^{2}
$$

Portanto, $(\alpha .68)$ resultou:

$$
\begin{aligned}
& E\left[S Q t^{\prime}\right]=n m^{2}+\tau^{*} U \tau *+u \sigma^{2}-n m^{2}-[1+(u-1) \rho] \sigma^{2} \\
&=(u-1) \sigma^{2}-(u-1) \rho \sigma^{2}+\tau^{*} U \tau^{*} \\
& \therefore E\left[S Q t^{\prime}\right]=(u-1)(1-\rho) \sigma^{2}+\tau^{*} \cdot U \tau^{*}
\end{aligned}
$$

e, obviamente

$$
\tau * ' U \tau *=r v \sum_{s=1}^{u} t_{s}^{2},
$$

obtendo-se, assim, em $(\alpha .70)$, um resultado idêntico àqueles encontrados na metodologia usual da anālise de variância para experimentos com parcelas subdivididas delineados em blocos (completos) casualizados, fornecida pelos autores que, como CHAKRABARTI (1962) adotam correlação constante entre subparcelas de uma mesma parcela.

De modo análogo aos anteriores:

$$
E\left[Q M t^{\prime}\right]=(1-\rho) \sigma^{2}+\frac{\tau *^{\prime} U \tau *}{u-1}
$$




\subsubsection{5 - Interação $t \times t^{\prime}$}

$$
\begin{aligned}
& \text { Reportando-se a }(\alpha .49) \text {, tomou-se } \\
& \qquad \mathrm{SQd}^{\prime}=\Delta^{\prime} \mathrm{L}^{-1} \Delta-\mathrm{C}_{0}-\tilde{\mathrm{T}}^{\prime} \mathrm{T}-\hat{\tau}^{*} \mathrm{~T}^{*} .
\end{aligned}
$$

Então,

$$
E[S Q \delta]=E\left[\Delta^{\prime} L^{-1} \Delta\right]-E\left[C_{\sigma}^{-}-E\left[\tilde{T}^{\prime} T\right]-E\left[\hat{T}^{\prime} T^{\prime}\right]-(\alpha .71)\right.
$$

com

i) $\Delta^{\prime} L^{-1} A=Y^{\prime} X_{5} L^{-1} X_{5}^{\prime} Y=\left(\theta^{\prime} X^{\prime}+\varepsilon^{\prime}\right) X_{5} L^{-1} X_{5}^{\prime}(X \theta+\varepsilon)$

$$
\therefore E\left[\Delta^{\prime} L^{-1} \Delta\right]=\theta^{\prime} X^{\prime} X_{5} L^{-1} X_{5}^{\prime} X \theta+E\left[\varepsilon^{\prime} X_{5} L^{-1} X_{5}^{\prime} \varepsilon\right]
$$

onde,

$$
\begin{gathered}
\text { i.1) } x_{5}=\left[\begin{array}{c}
I \text { (uv) } \\
I \text { (uv) } \\
\ldots \\
I_{\text {(uv) }}
\end{array}\right] \quad \text { matriz I (uv) para cada repetição, } \\
\text { (urv) (urv) }
\end{gathered}
$$

então, de modo análogo ao desenvolvido na obtenção de ( $\alpha .69)$, obteve-se

$$
E\left[\varepsilon^{\prime} X_{5} L^{-1} X_{5}^{\prime} \varepsilon\right]=u v \sigma^{2}
$$


i. 2) $\quad X^{\prime} X_{5} L^{-1} X_{5}^{\prime} X=\left[\begin{array}{ccccc}n & x & y & z & w \\ x^{\prime} & R & N & P & S \\ y^{\prime} & N^{\prime} & N^{\prime} R^{-1} N & K^{\prime} & V \\ z^{\prime} & P^{\prime} & K^{\prime} & U & H \\ w^{\prime} & S^{\prime} & V^{\prime} & H^{\prime} & L\end{array}\right]$

Assim, dadas as restrições citadas em 4.2, resultou

$$
\begin{aligned}
\theta^{\prime} X^{\prime} X_{5} L^{-1} X_{5}^{\prime} X \theta=n m^{2} & +\tau^{\prime} R \tau+\beta^{\prime} N^{\prime} \tau+\tau^{\prime} N B+B^{\prime} N^{\prime} R^{-1} N B+ \\
& +\tau^{*} U \tau^{*}+\delta^{\prime} H^{\prime} \tau^{*}+\tau^{\prime} H \delta+\delta^{\prime} L \delta
\end{aligned}
$$

$\therefore E\left[\Delta^{\prime} L^{-1} \Delta\right]=u v \sigma^{2}+C_{0}+\tau^{\prime} R \tau+B^{\prime} N^{\prime} \tau+\tau^{\prime} N B+B^{\prime} N^{\prime} R^{-1} N B+$

$$
+\tau *^{\prime} U \tau *+\delta^{\prime} H^{\prime} \tau^{*}+\tau^{*} H \delta+\delta^{\prime} L \delta(\alpha .73)
$$

ii) $\tilde{\tau}^{\prime} \mathrm{T}$

$$
\begin{aligned}
& \text { Usando-se o fato de que por }(\alpha .48 . i) \text {, } \\
& \qquad \tilde{\tau}^{\prime} T+C_{0}=T^{\prime} R^{-1} S L^{-1} \Delta,
\end{aligned}
$$

obteve-se

$$
\tilde{\tau}^{\prime} T=T^{\prime} R^{-1} S L^{-1} \Delta-C_{0},
$$

onde $\mathrm{SL}^{-1} \Delta=\mathrm{T}$,

$$
\begin{aligned}
\therefore \quad \tilde{\tau}^{\prime} T & =T^{\prime} R^{-1} T-C_{0} \\
& =Y^{\prime} X_{2} R^{-1} X_{2}^{\prime} Y-C_{0} \\
& =\left(\theta^{\prime} X^{\prime}+\varepsilon^{\prime}\right) X_{2} R^{-1} X_{2}^{\prime}(X \theta+\varepsilon)-C_{0}
\end{aligned}
$$




$$
\therefore \quad E\left[\tilde{T}^{\prime} T\right]=\theta^{\prime} X^{\prime} X_{2} R^{-1} X_{2}^{\prime} X \theta+E\left[\varepsilon^{\prime} X_{2} R^{-1} X_{2}^{\prime} \varepsilon\right]-E\left[C_{0}\right] \quad(0.74)
$$

onde:

ii.1) $E\left[\varepsilon^{\prime} X_{2} R^{-1} X_{2}^{\prime} \varepsilon\right]=\operatorname{TR}\left[R^{-1} x_{2}^{\prime} X_{2}\right] \cdot[1+(u-1) p] \sigma^{2}$

$$
=\operatorname{TR}\left[I_{(v)}\right] \cdot[1+(u-1) p] \sigma^{2}
$$

$E\left[\varepsilon^{\prime} X_{2} R^{-1} X_{2}^{\prime} \varepsilon\right]=v[1+(u-1) \quad p] \sigma^{2}$

$i i .2) \quad x^{\prime} x_{2} R^{-1} x_{2}^{\prime} X=\left[\begin{array}{ccccc}x R^{-1} x^{\prime} & x & x R^{-1} N & x R^{-1} P & x R^{-1} S \\ x & R & N & P & S \\ N^{\prime} R^{-1} x^{\prime} & N^{\prime} & N^{\prime} R^{-1} N & N^{\prime} R^{-1} P & N^{\prime} R^{-1} S \\ P^{\prime} R^{-1} x^{\prime} & P^{\prime} & P^{\prime} R^{-1} N & P^{\prime} R^{-1} P & P^{\prime} R^{-1} S \\ S^{\prime} R^{-1} x^{\prime} & S^{\prime} & S^{\prime} R^{-1} N & S^{\prime} R^{-1} P & S^{\prime} R^{-1} S\end{array}\right]$

$\therefore \theta^{\prime} X^{\prime} X_{2} R^{-1} X_{2}^{\prime} X \theta=n m^{2}+\tau^{\prime} R \tau+B^{\prime} N^{\prime} \tau+\tau^{\prime} N B+B^{\prime} N^{\prime} R^{-1} N B$

$\therefore E\left[\tilde{\tau}^{\prime} T\right]=(v-1)[1+(u-1) \rho] \sigma^{2}+\tau^{1} \hbar \tau+$

$$
+2 B^{\prime} N^{\prime} \tau+B^{\prime} N^{\prime} R^{-1} N B
$$

iii) $\hat{\tau}^{*} \mathrm{~T}^{*}=\mathrm{T}^{*} \mathrm{U}^{-1} \mathrm{~T} *-\hat{\mathrm{m}} \mathrm{U}^{-1} \mathrm{zT} * \quad($ por $\alpha .13)$

$$
=\mathrm{T} * \mathrm{U}^{-1} \mathrm{~T} *-\mathrm{Co}_{0} \text {. }
$$

Então, usando-se $(4.6 .2 .2 .4)$ e $(\alpha .70)$, obteve-se

$$
E\left[\hat{\tau}^{\prime} T^{*}\right]=E\left[S Q T^{\prime}\right]=(u-1)(1-p) \sigma^{2}+\tau^{*} U \tau^{*}(\alpha .76 *)
$$

Desse modo, $(\alpha .71)$ passou a ser, por $(\alpha .73),(\alpha .57)$, $(\alpha .76)$ e $(\alpha .76 *)$ 


$$
\begin{aligned}
& E[S Q \delta]=(u-1)(v-1)(1-\rho) \sigma^{2}+\delta^{\prime} L \delta \\
\therefore \quad & E[Q M \delta]=(1-\rho) \sigma^{2}+\frac{1}{(u-1)(v-1)} \delta^{\prime} L \delta
\end{aligned}
$$

4.6.3 - Soma de quadrados devida às parcelas

$$
\begin{aligned}
& \text { Tomando-se como em }(\alpha .45 \cdot *) \\
& \qquad S Q \operatorname{Parc}=\frac{1}{u} Y^{\prime} \Phi Y-C_{0}
\end{aligned}
$$

resultou

$$
\begin{gathered}
E[\text { SQParc }]=\frac{1}{u} E\left[Y^{\prime} \Phi Y\right]-E\left[C_{O}\right] \\
\therefore E[S Q P a r C]=\frac{1}{u}\left\{\theta^{\prime} X^{\prime} \Phi X \theta+E\left[E^{\prime} \Phi \varepsilon\right]\right\}-E\left[C_{O}\right]
\end{gathered}
$$

i) Analisando-se o termo $E\left[\varepsilon^{\prime} \phi \varepsilon\right]$, obteve-se, sem perda de generali dade, para a primeira parcela

$$
\left[e_{111}, e_{112}, \ldots, e_{11 u}\right]\left[\begin{array}{ccc}
1 & \ldots & 1 \\
1 & \ldots & 1 \\
\ldots & \ldots & \ldots \\
1 & \ldots & 1
\end{array}\right]\left[\begin{array}{l}
e_{111} \\
e_{112} \\
\ldots \\
e_{114}
\end{array}\right]
$$

então, em termos de esperanças, para essa parcela, resultou

$$
u \sigma^{2}+u(u-1) \rho \sigma^{2}
$$

assim, considerando-se o comportamento análogo para as rv parcelas do experimento:

$$
E\left[E^{\prime} \Phi \varepsilon\right]=\operatorname{urv}[1+(u-1) \rho] \sigma^{2}
$$


ii) No tocante ao termo $\theta^{\prime} X^{\prime} \Phi X \theta$, obteve-se

$$
X^{\prime} \Phi X=\left[\begin{array}{ccccc}
u n & u x & u y & u z & u w \\
u x^{\prime} & u R & u N & u P & u S \\
u y^{\prime} & u N^{\prime} & u A & u K & u V \\
u z^{\prime} & u P^{\prime} & u K^{\prime} & U^{*} & H \\
u w^{\prime} & u S^{\prime} & u V^{\prime} & H^{\prime} & L^{*}
\end{array}\right]
$$

onde

$$
U^{*}=r v_{(u)}{ }^{E}(u)
$$

então $\tau *^{*} U *_{\tau}^{*}=\Phi, e$

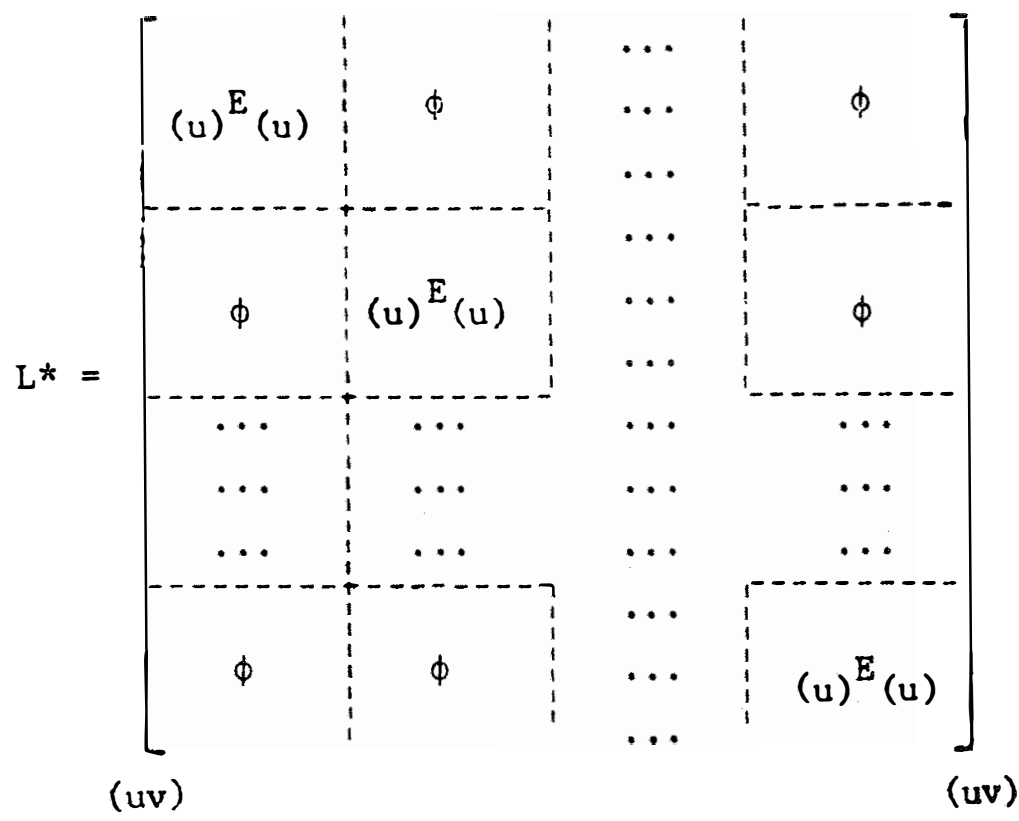

resultando $\delta^{\prime} L * \delta=\phi$.

Então,

$$
\theta^{\prime} X^{\prime} \Phi X \theta=u\left(n m^{2}+\tau^{\prime} R \tau+B^{\prime} A B+2 B^{\prime} N^{\prime} \tau\right) .
$$


Obteve-se, desse modo, para $(\alpha .78)$

$E[S Q P a r c]=\frac{1}{u}\left\{u n m^{2}+u \tau^{\prime} R \tau+u B^{\prime} A B+2 u B^{\prime} N^{\prime} \tau+u r v[1+(u-1) \rho] \sigma^{2}\right\}-$

$$
-\mathrm{nm}^{2}-[1+(u-1) \rho] \sigma^{2}
$$

$\therefore E[S Q P a r c]=(r v-1)[1+(u-1) \rho] \sigma^{2}+\tau^{\prime} R \tau+B^{\prime} A B+$

$$
+2 B^{\prime} N^{\prime} \tau
$$

$\therefore E[Q M P a r c]=[1+(u-1) \quad p] \sigma^{2}+\frac{1}{r v-1}\left\{\begin{array}{c}\tau^{\prime} R \tau+B^{\prime} A B+2 B^{\prime} N^{\prime} \tau\end{array}\right\}$

4.6.4 - Soma de quadrados devida ao total

$$
S Q T=Y^{\prime} Y-C_{O}
$$

onde $Y^{\prime} Y=Y^{\prime} \Phi Y+Y^{\prime}(I-\Phi) Y$

$$
\begin{aligned}
\therefore E[S Q T]= & E\left[Y^{\prime} \Phi Y\right]+E\left[Y^{\prime}(I-\Phi) Y\right]-E\left[C_{\sigma}\right] \\
= & \theta^{\prime} X^{\prime} \Phi X \theta+E\left[\varepsilon^{\prime} \Phi \varepsilon\right]+\theta^{\prime} X^{\prime}(I-\Phi) X \theta+ \\
& +E\left[\varepsilon^{\prime}(I-\Phi) \varepsilon\right]-E\left[C_{\sigma}\right],
\end{aligned}
$$

com

i) $\theta^{\prime} X^{\prime} \Phi X \theta+\theta^{\prime} X^{\prime}(I-\Phi) X \theta=\theta^{\prime} X^{\prime} X \theta=n m^{2}+\tau^{\prime} R \tau+B^{\prime} A B+2 B^{\prime} N^{\prime} \tau+$

$$
+\tau^{*} U \tau *+\delta^{\prime} L \delta
$$

ii) $E\left[\varepsilon^{\prime} \Phi \varepsilon\right]=\operatorname{urv}[1+(u-1) \rho] \sigma^{2}$

iii) $E\left[\varepsilon^{\prime}(I-\Phi) \varepsilon\right]=-\operatorname{urv}(u-1) \rho \sigma^{2}$, pois, com raciocínio análogo 
ao desenvolvido em (4.6.3.i), obteve-se para a primeira parcela

$$
\left[e_{111}, e_{112}, \ldots, e_{11 u}\right]\left[\begin{array}{cccc}
0 & -1 & \ldots & -1 \\
-1 & 0 & \ldots & -1 \\
\ldots & \ldots & \ldots & \ldots \\
-1 & -1 & \ldots & 0
\end{array}\right]\left[\begin{array}{l}
e_{111} \\
e_{112} \\
\ldots \\
e_{11}
\end{array}\right]
$$

(u)

(u)

então, em termos de esperança:

$$
-u(u-1) \rho \sigma^{2}
$$

E, para as rv parcelas do ensaio

$$
E\left[\begin{array}{ll}
\varepsilon^{\prime}(I-\Phi) & \varepsilon
\end{array}\right]=-\operatorname{urv}(u-1) \rho \sigma^{2}
$$

De fato,

$\operatorname{urv}[1+(u-1) \rho] \sigma^{2}-\operatorname{urv}(u-1) \rho \sigma^{2}=\operatorname{urv} \sigma^{2}=E\left[\varepsilon^{\prime} \varepsilon\right]$

Assim, resultou

$$
\begin{aligned}
E[\mathrm{SQT}]= & \operatorname{urv} \sigma^{2}-[1+(u-1) \rho] \sigma^{2}+\tau^{\prime} R \tau+ \\
& +\beta^{\prime} A B+2 B^{\prime} N^{\prime} \tau+\tau^{\prime} \cdot U \tau+\delta^{\prime} L \delta
\end{aligned}
$$

ou

$$
\begin{aligned}
E[\mathrm{SQT}]= & {[(\mathrm{urv}-1)-(u-1) \rho] \sigma^{2}+\tau^{\prime} \mathrm{R} \tau+B^{\prime} \mathrm{A} \beta+} \\
& +2 B^{\prime} \mathrm{N}^{\prime} \tau+\tau^{*} \mathrm{U} \tau^{*}+\delta^{\prime} L \delta
\end{aligned}
$$



4.6.5 - Componentes de variâncias devidos aos efeitos alea tōrios

$$
\text { 4.6.5.1 - Residuo (a) }
$$

$$
\operatorname{SQRes}(a)=S Q P a r c-S Q t(a j)-S Q b
$$

$$
\begin{aligned}
\therefore \quad E[\operatorname{SQRes}(a)]= & E[\operatorname{SQParc}]-E[\operatorname{SQt}(a j)]-[\operatorname{SQb}] \\
= & (\mathrm{rv}-1)[1+(\mathrm{u}-1) \rho] \sigma^{2}+\tau^{\prime} R \tau+ \\
& +\beta^{\prime} A B+2 B^{\prime} N^{\prime} \tau-(\mathrm{v}-1)[1+(\mathrm{u}-1) \rho] \sigma^{2}- \\
& -\tau^{\prime} Q-(\mathrm{a}-1)[1+(\mathrm{u}-1) \rho] \sigma^{2}- \\
& -\tau^{\prime} R \tau+\tau^{\prime} Q-2 B^{\prime} N^{\prime} \tau-B^{\prime} A B
\end{aligned}
$$

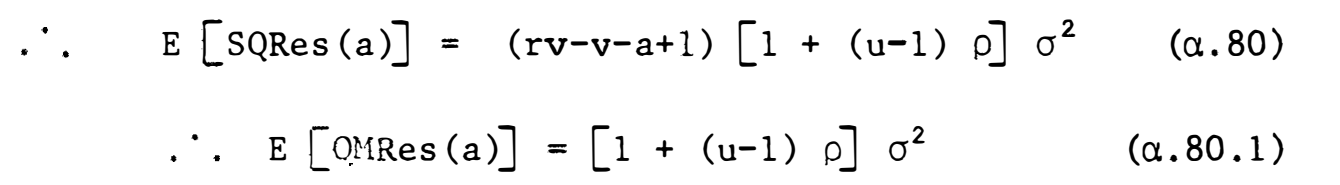

$$
4.6 .5 .2 \text { - Residuo (b) }
$$

$$
\text { SQRes }(b)=S Q T-S Q P a r c-S Q t^{\prime}-S Q \delta
$$

$\therefore \quad \mathrm{E}[\operatorname{SQRes}(\mathrm{b})]=\mathrm{E}[\mathrm{SQT}]-\mathrm{E}[\mathrm{SQParc}]-\mathrm{E}\left[\mathrm{SQt} \mathrm{t}^{\prime}\right]-\mathrm{E}[\mathrm{SQ} \delta]$

$$
\begin{aligned}
= & {[(u r v-1)-(u-1) \rho] \sigma^{2}+\tau^{\prime} R \tau+\beta^{\prime} A B+} \\
& +2 \beta^{\prime} N^{\prime} \tau+\tau^{\prime} U \tau^{*}+\delta^{\prime} L \delta- \\
& -(r v-1)[1+(u-1) \rho] \sigma^{2}-\tau^{\prime} R \tau-\beta^{\prime} A B- \\
& -2 \beta^{\prime} N^{\prime} \tau-(u-1)(1-\rho) \sigma^{2}-\tau^{\prime} U \tau^{*}- \\
& -(u-1)(v-1)(1-\rho) \sigma^{2}-\delta^{\prime} L \delta
\end{aligned}
$$




$$
\begin{aligned}
\therefore \mathrm{E}[\operatorname{SQRes}(b)]= & (u r v-1-r v+1-u+1-u v+v+u-1) \sigma^{2}+ \\
& +(-u+1-u r v+u+r v-1+u-1+u v-v-u+1) \rho \sigma^{2} \\
= & v(u-1)(r-1) \sigma^{2}-v(u-1)(r-1) \rho \sigma^{2} \\
\therefore E[\operatorname{SQRes}(b)]= & v(u-1)(r-1)[1-\rho] \sigma^{2}
\end{aligned}
$$

Assim, ficou

$$
E[Q \operatorname{Res}(b)]=(1-\rho) \sigma^{2}
$$

4.6.6 - Testes de hipōteses e quadros de anāilise

Conforme citado em (2.1.4), nos ensaios com parcelas subdivididas existe geralmente interesse em se testar as três hipóteses bāsicas:

$$
\begin{aligned}
& H_{0}(1): \quad t_{i}=0 ; i=1,2, \ldots, v \\
& H_{0}(2): t_{s}^{\prime}=0 ; \quad s=1,2, \ldots, u \\
& H_{0}(3): \delta_{i s}=0 ; i=1,2, \ldots, v ; s=1,2, \ldots, u,
\end{aligned}
$$

que são, respectivamente, as hipóteses de nulidade para efeitos de tratamentos principais, efeitos de tratamentos secundários e efeitos de interação.

Os erros adequados para cada teste, ficaram evidentes quando se observou a coluna relativa às esperanças dos quadrados médios, no quadro da análise de variância: 


\begin{tabular}{|c|c|c|c|}
\hline $\begin{array}{l}\text { Causas da } \\
\text { Variação }\end{array}$ & $\begin{array}{l}\text { Graus de } \\
\text { liberdade }\end{array}$ & $\begin{array}{l}\text { Somas de } \\
\text { quadrados }\end{array}$ & $\begin{array}{l}\text { Esperança dos } \\
\text { quadrados médios* }\end{array}$ \\
\hline Blocos & $a-1$ & $B^{\prime} A^{-1} B-C_{O}$ & {$[1+(u-1) \rho] \sigma^{2}+f_{1}(\theta)$} \\
\hline Trat $(a j) t$. & $v-1$ & $\hat{\tau}^{\prime} Q$ & {$[1+(\mathrm{u}-1) \rho] \sigma^{2}+\mathrm{f}_{2}(\theta)$} \\
\hline Resíduo (a) & $r v-v-a+1$ & DIFERENÇA & {$[1+(u-1) \rho] \sigma^{2}$} \\
\hline Parcelas & $\mathrm{rv}-1$ & $\frac{1}{\mathrm{u}} \mathrm{Y}^{\prime} \Phi \mathrm{Y}-\mathrm{C}_{\mathrm{O}}$ & \\
\hline Trat. $t^{\prime}$ & $u-1$ & $T * U^{-1} T *-C_{0}$ & $(1-\rho) \sigma^{2}+f_{3}(\theta)$ \\
\hline$t \times t^{\prime}$ & $(u-1)(v-1)$ & $\Delta^{\prime} L^{-1} \Delta-C_{0}-S Q t-S Q t^{\prime}$ & $(1-\rho) \sigma^{2}+f_{4}(\theta)$ \\
\hline Resíduo & $v(u-1)(r-1)$ & DIFERENÇA & $(1-\rho) \sigma^{2}$ \\
\hline Total & urv-1 & $\mathrm{Y}^{\prime} \mathrm{Y}-\mathrm{C}_{\mathrm{O}}$ & \\
\hline
\end{tabular}

(*) $\mathrm{f}_{1}(\theta)=\left\{B^{\prime} \mathrm{A} B+\tau^{\prime} \mathrm{R} \tau-\tau^{\prime} Q-2 B^{\prime} \mathrm{N}^{\prime} \tau\right\} /(\mathrm{a}-1)$

$\mathrm{f}_{2}(\theta)=\left\{\tau^{\prime} \mathrm{Q}\right\} /(\mathrm{v}-1)$

$\mathrm{f}_{3}(\theta)=\left\{\tau *^{\prime} \mathrm{U} \tau *\right\} /(\mathrm{u}-1)$

$\mathrm{f}_{4}(\theta)=\left\{\delta^{\prime} \mathrm{L} \delta\right\} /(\mathrm{u}-1)(\mathrm{v}-1)$

Então, no tocante às três hipóteses bāsicas, o expe rimento com parcelas subdivididas, com tratamentos principais dispos tos em blocos incompletos balanceados, comportou-se de de modo análo go ao seu equivalente em blocos (completos) casualizados.

Assim, ficaram determinados os critérios para os res pectivos testes:

\begin{tabular}{ccc}
\hline Hipóteses & G.L. & F (observado) \\
\hline $\mathrm{H}_{\mathrm{O}}(1)$ & $(\mathrm{v}-1) ;(\mathrm{rv}-\mathrm{v}-\mathrm{a}+1)$ & QMt (aj .)/QMRes (a) \\
$\mathrm{H}_{\mathrm{O}}(2)$ & $(\mathrm{u}-1) ;[\mathrm{v}(\mathrm{u}-1)(\mathrm{r}-1)]$ & QMt ' / QMRes (b) \\
$\mathrm{H}_{\mathrm{O}}(3)$ & $(\mathrm{u}-1)(\mathrm{v}-1) ;[\mathrm{v}(\mathrm{u}-1)(\mathrm{r}-1)]$ & QMo /QMRes (b) \\
\hline
\end{tabular}


No entanto, se for desejävel testar-se uma hipötese adicional, relativa aos efeitos de blocos, então o esquema incomple to passa a ser o agente diferenciador, e o tipo do BIB em questão de ve ser considerado.

Sob esse aspecto, como citado em (2.2.4), PIMENTEL GOMES (1976), dentre outros, recomenda a decomposição da soma de quadrados de blocos conforme o BIB seja do tipo I ou do tipo II, do seguinte modo:

$$
\begin{aligned}
& \text { TIPO I: SQBlocos = SQRepetições + SQBlocos/Repetições } \\
& \text { TIPO II: SQBlocos = SQGrupos + SQBlocos/Grupos }
\end{aligned}
$$

com:

$$
\begin{aligned}
\text { SQRepetições } & =\frac{1}{v} \sum_{d} R_{d}^{2}-C_{0} \\
\text { SQGrupos } & =\frac{1}{f \cdot v_{g}} \sum_{g}^{2}-C_{0}
\end{aligned}
$$

onde, $R_{d}, d=1, \ldots, f, \ldots, r$ representa o total da $d$-ésima repetição; $G_{g}^{a} ; g=1, \ldots, h$ representa o total do g-ésimo grupo de repetições;

f representa o nümero de repetições por grupo.

Neste estudo, como cada tratamento principal está ainda repetido em $\underline{u}$ subparcelas, adotou-se 


$$
\begin{aligned}
& \text { SQRepetições }=\frac{1}{u v} \sum_{d} R_{d}^{2}-C_{0}
\end{aligned}
$$

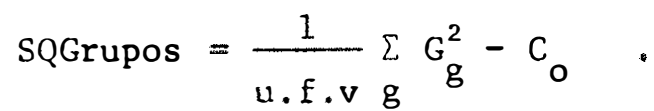

Para os testes das hipóteses adicionais,

$$
H_{0}(4): \quad b_{p} / R_{d}=0 ; p=1, \ldots, a^{*} ; d=1, \ldots, r
$$

onde, $a^{*}=\frac{a}{r}$ é o número de blocos por repetição,

$$
H_{0}(5): \quad b_{p^{\prime}} / G_{g}=0 ; p^{\prime}=1, \ldots, a^{*^{\prime}} ; g=1, \ldots, h
$$

onde $\mathrm{a}^{*^{\prime}}=\frac{\mathrm{a}}{\mathrm{h}}$ é o número de blocos por grupo.

Tomou-se o resíduo (a) como polo de referência, resultando os seguintes critérios:

\begin{tabular}{ccc}
\hline Hipóteses & G.L. & F (observado) \\
\hline $\mathrm{H}_{\mathrm{O}}(4)$ & $(\mathrm{a}-\mathrm{r}) ;(\mathrm{rv}-\mathrm{v}-\mathrm{a}+1)$ & $(\mathrm{QMb} / \operatorname{Rep}) /[\mathrm{QMRes}(\mathrm{a})]$ \\
$\mathrm{H}_{\mathrm{O}}(5)$ & $(\mathrm{a}-\mathrm{h}) ;(\mathrm{rv}-\mathrm{v}-\mathrm{a}+1)$ & $(\mathrm{QMb} / \mathrm{Gru}) /[\mathrm{QMRes}(\mathrm{a})]$ \\
\hline
\end{tabular}

que corresponderam exatamente aos resultados apresentados por PIMEN TEL GOMES (1976) para ensaios em blocos incompletos balanceados.

De modo análogo, pode haver interesse em se testar, ainda, efeitos de repetições e efeitos de grupos

$$
\begin{array}{ll}
H_{0}(6): R_{d}=0 ; & d=1, \ldots, r \\
H_{0}(7): & G_{g}=0 ; g=1, \ldots, h
\end{array}
$$


Para essas hipóteses foram obtidos os seguintes critérios:

\begin{tabular}{ccc}
\hline Hipóteses & G.L. & F (observado) \\
\hline$H_{0}(6)$ & $(r-1) ;(r v-v-a+1)$ & QMRep/QMRes (a) \\
$H_{0}(7)$ & $(g-1) ;(r v-v-a+1)$ & QMGru/QMRes (a) \\
\hline
\end{tabular}

De fato, vejamos como foram obtidos os critérios para o experimento citado no exemplo inicial (BIB tipo I), e de modo análogo, os critérios para o teste de $H_{0}(5)$ e $H_{0}(7)$, de um experimento com parcelas subdivididas em BIB tipo II,

$$
\text { SQRep } \left.=\frac{1}{u v} \sum_{d} R_{d}^{2}-C_{0} . \quad \text { (por } \alpha .82\right)
$$

Como as hipóteses de maior interesse em ensaios do tipo 'Split-plot"sao, geralmente, relacionadas aos tratamentos principais, $H_{0}(1)$, aos tratamentos secundários, $H_{0}(2)$, e à interação, $H_{0}(3)$, não se cogitou em acrescentar ao modelo matemático descrito em (4.2), parâmetros relativos às repetições ou grupos.

$$
\text { Supôs-se, no entanto, para os testes de } \mathrm{H}_{\mathrm{o}}(4) \text { e. } \mathrm{H}_{\mathrm{o}}(6) \text {, }
$$
a existência do parâmetro $R_{d}$, relativo à d-ésima repetição.

Sob esse aspecto, à $R_{d}$ correspondeu a submatriz $x_{6}$, que foi anexada à já existente matriz $x$ do modelo citado em (4.2), resultando 


$$
\mathrm{x}^{*}=\left[\mathrm{x}_{1}: \mathrm{x}_{2}: \mathrm{x}_{3}: \mathrm{x}_{4}: \mathrm{x}_{5}: \mathrm{x}_{6}\right]
$$

onde, $x_{1}, x_{2}, x_{5}, x_{4}$ e $x_{5}$ continuaram sendo as mesmas submatrizes de $(4.3), e$

$$
x_{6}=\left[\begin{array}{cccc}
1 & 0 & \ldots & 0 \\
\ldots & \ldots & \ldots & \ldots \\
1 & 0 & \ldots & 0 \\
0 & 1 & \ldots & 0 \\
\ldots & \ldots & \ldots & \ldots \\
0 & 1 & \ldots & 0 \\
\ldots & \ldots & \ldots & \ldots \\
\ldots & \ldots & \ldots & \ldots \\
0 & 0 & \ldots & 1 \\
\ldots & \ldots & \ldots & \ldots \\
0 & 0 & \ldots & 1
\end{array}\right]
$$

Através do uso de $X_{6},(\alpha .82)$ passou a ser

$$
\text { SQRep }=\frac{1}{u v} R^{*} R *-C_{0},
$$

onde $R *=X_{6}^{1} Y$, então

$$
\begin{aligned}
E[\text { SQRep }] & =\frac{1}{u v} E\left[Y^{\prime} X_{6} X_{6}^{\prime} Y\right]-E\left[C_{0}\right] \\
& =\frac{1}{u v}\left\{\theta^{\prime} X^{\prime} X_{6} X_{6}^{\prime} X \theta+E\left[\varepsilon^{\prime} X_{6} X_{6}^{\prime} \varepsilon\right]\right\}-E\left[C_{0}\right]
\end{aligned}
$$




$$
\therefore E[\operatorname{SQRep}]=\frac{1}{u v} f(\theta)+\frac{1}{u v} E\left[\varepsilon^{\prime} X_{6} X_{6}^{\prime} \varepsilon\right]-E\left[C_{0}\right], \quad(\alpha .84)
$$

onde $f(\theta)$ é uma função não negativa dos parâmetros, como as descritas em 4.6.6.

$$
\text { No tocante à parte aleatória, obteve-se }
$$

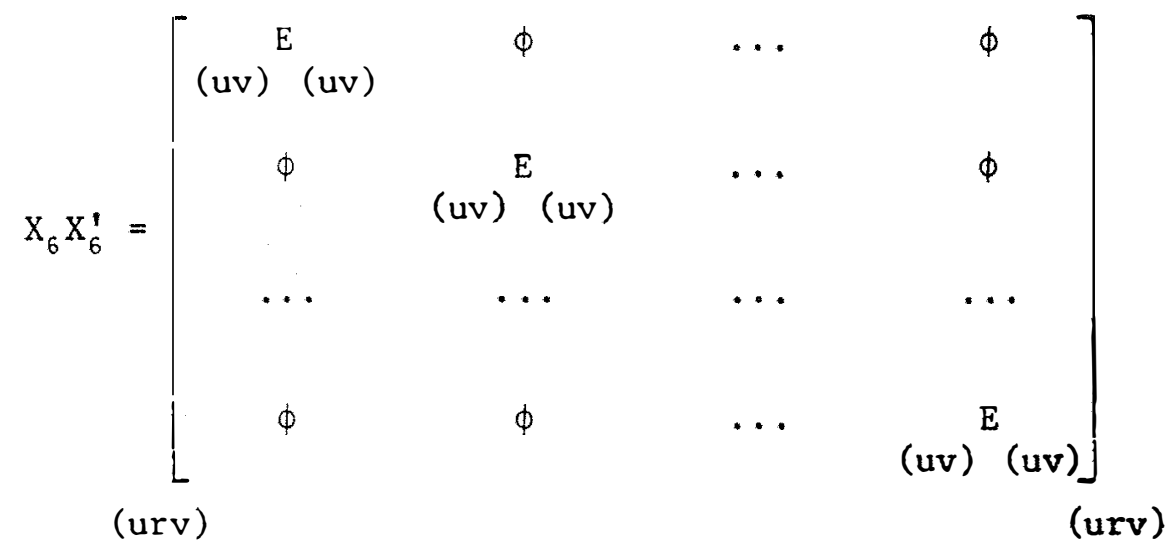

onde a cada repetição correspondeu uma submatriz (uv) (uv)

Assim, para a primeira repetição, sem perda de gene-

ralidade, foi obtido:

$$
\left[\begin{array}{lllllllll}
e_{111} & \cdots & e_{11 u} & e_{211} & \cdots & e_{214} & \cdots & \cdots & e_{v a * u}
\end{array}\right]\left[\begin{array}{ccc}
1 & \ldots & 1 \\
\vdots & \cdots & \vdots \\
1 & \ldots & 1
\end{array}\right]\left[\begin{array}{c}
e_{111} \\
\vdots \\
e_{v a * u}
\end{array}\right]
$$

que, em termos de esperança, resultou

$$
\mathrm{ua} * \mathrm{k}[1+(\mathrm{u}-1) 0] \sigma^{2}=\mathrm{u} \frac{\mathrm{a}}{\mathrm{r}} \mathrm{k}[1+(\mathrm{u}-1) \mathrm{j}] \sigma^{2}
$$

e, desse modo, para as $\underline{r}$ repetições do experimento, onde ak $=r v$ 


$$
E\left[\varepsilon^{\prime} x_{6} x_{6}^{\prime} \varepsilon\right]=u r v[1+(u-1) \rho] \sigma^{2}
$$

então, a parte aleatória de $(\alpha .84)$, ficou:

$$
\begin{aligned}
& \frac{1}{u v} E\left[\varepsilon^{\prime} X_{6} X_{6}^{\prime} \varepsilon\right]-E\left[C_{\sigma}\right]=(r-1)[1+(u-1) \rho] \sigma^{2} \\
\therefore \quad & E[\operatorname{SQRep}]=(r-1)[1+(u-1) \rho] \sigma^{2}+f(\theta) \quad(\alpha .84 .1)
\end{aligned}
$$

e

$$
E[\mathrm{QMRep}]=[1+(\mathrm{u}-1) \rho] \sigma^{2}+\frac{1}{\mathrm{r}-1} \mathrm{f}(\theta) \quad(\alpha .84 .2)
$$

tornando-se, assim, justificado o critério adotado para o teste de $H_{0}(6)$

Tomando-se, então, como em PIMENTEL GOMES (1976)

$$
\mathrm{SQb} / \operatorname{Rep}=\mathrm{SQb}-\mathrm{SQReP},
$$

obteve-se

$$
\begin{aligned}
E[\mathrm{SQb} / \mathrm{Rep}]=(\mathrm{a}-1)[1 & +(\mathrm{u}-1) \rho] \sigma^{2}+\mathrm{f}_{1}(\theta) \\
& -(\mathrm{r}-1)[1+(\mathrm{u}-1) \rho] \sigma^{2}-\mathrm{f}(\theta)
\end{aligned}
$$$$
\therefore \quad E[S Q b / \operatorname{Rep}]=(a-r)[1+(u-1) \rho] \sigma^{2}+f *(\theta) \cdot(\alpha .85)
$$

Obviamente, em se tratando de BIB, ocorre sempre a>r e, desse modo:

$$
E[\mathrm{QMb} / \mathrm{Rep}]=[1+(\mathrm{u}-1) \rho] \sigma^{2}+\frac{1}{\mathrm{a}-\mathrm{r}} \mathrm{f} *(\theta)
$$

comprovando-se, assim, a validade do teste para $\mathrm{H}_{\mathrm{O}}(4)$.

Então, para ensaios com parcelas subdivididas com tra tamentos principais dispostos em blocos incompletos balanceados do 
tipo I, para os testes de $\mathrm{H}_{0}(1), \mathrm{H}_{0}(2), \mathrm{H}_{0}(3), \mathrm{H}_{0}(4)$ e $\mathrm{H}_{0}(6)$, obteve-se o seguinte quadro de anālise:

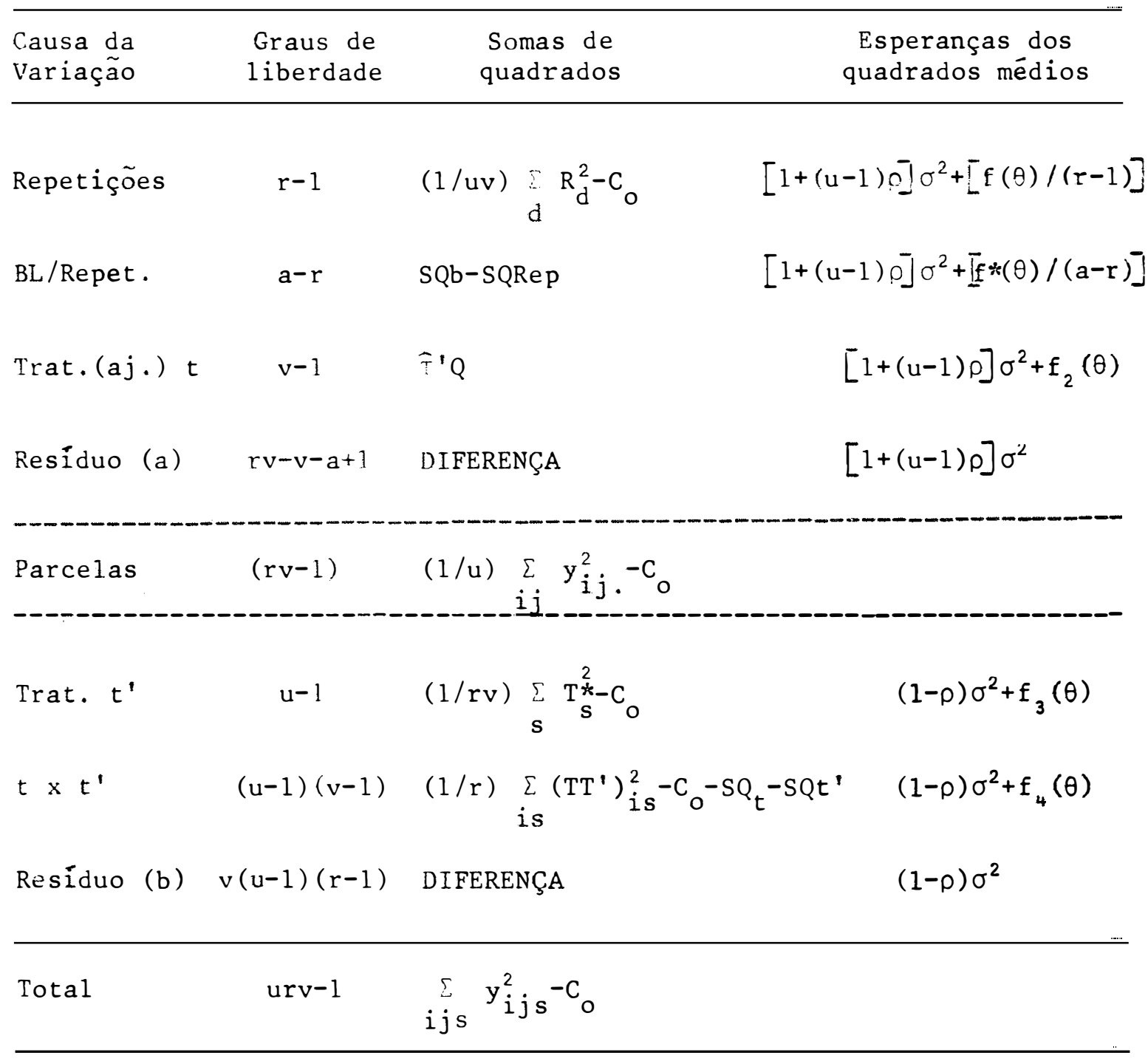

no qual observou-se que a "parte superior", correspondente aos tratamentos principais, comportou-se como numa análise de experimentos em blocos incompletos balanceados. Por sua vez, a "parte inferior", correspondente aos tratamentos secundários, portou-se como num en- 
saio em parcelas subdivididas em blocos (completos) casualizados, in

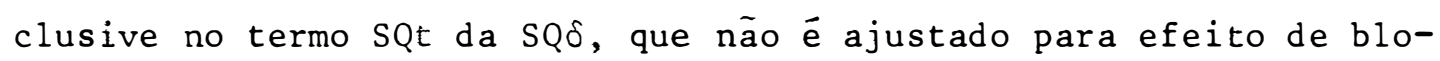
cos.

De modo análogo, obteve-se o quadro da análise da va riância para o caso de "Split-plot" em BIB tipo II, que mostrou-se idêntico ao caso anterior, com exceção do desdobramento da SQblocos, resultando então, para a "parte superior":

\begin{tabular}{|c|c|c|c|}
\hline $\begin{array}{l}\text { Causa da } \\
\text { Variação }\end{array}$ & $\begin{array}{l}\text { Graus de } \\
\text { liberdade }\end{array}$ & $\begin{array}{l}\text { Somas de } \\
\text { quadrados }\end{array}$ & $\begin{array}{l}\text { Esperanças dos } \\
\text { quadrados médios }\end{array}$ \\
\hline Grupos & $g-1$ & $(1 / u \cdot f \cdot v) \sum_{g} G_{g}^{2}-C_{0}$ & {$[1+(u-1) \rho] \sigma^{2}+f *(\theta)$} \\
\hline BL/Grupos & $a-g$ & SQb-SQGru & {$[1+(u-1) \rho] \sigma^{2}+f_{s}(\theta)$} \\
\hline Trat. $(a j \cdot) t$ & $v-1$ & $\hat{\tau}^{\prime} Q$ & {$[1+(u-1) \rho] \sigma^{2}+f_{2}(\theta)$} \\
\hline Resíduo (a) & $r v-v-a+1$ & DIFERENÇA & {$[1+(u-1) \rho] \sigma^{2}$} \\
\hline Parcelas & $\mathrm{rv}-1$ & $(1 / u) \sum_{i j} y_{i j}^{2} \cdot-C_{0}$ & \\
\hline
\end{tabular}

onde se verificaram os critérios adotados para os testes de $\mathrm{H}_{\mathrm{O}}(5)$ e $\mathrm{H}_{\mathrm{O}}(7)$.

Por outro lado, segundo PIMENTEL GOMES (1976) e LEAL (1979), dentre outros, quando a interação tt' é significativa, o es quema da análise de variância deve ser modificado,pois este fato po de ser um indicio de que os tratamentos principais comportam-se de 
modos diferentes em relação aos tratamentos secundärios. Assim, recomendam que seja estudado o efeito dos tratamentos secundārios den tro de cada tratamento principal, isoladamente.

Sob esse aspecto, se $\mathrm{H}_{\mathrm{o}}$ (3) resulta significativa, en tão $H_{0}(2)$ deve ser decomposta em $\underline{v}$ subhipöteses do tipo:

$$
\begin{array}{cc}
H_{0}(2.1): & t^{\prime} / t_{1}=0 \\
H_{0}(2.2): & t^{\prime} / t_{2}=0 \\
\ldots & \cdots \\
H_{0}(2 . v): & t^{\prime} / t_{v}=0
\end{array}
$$

com o seguinte critério para os respectivos testes:

\begin{tabular}{ccc}
\hline Hipóteses & G.L. & F (observado) \\
\hline $\mathrm{H}_{\mathrm{O}}(2.1)$ & $(1) ;[\mathrm{v}(\mathrm{u}-1)(\mathrm{r}-1)]$ & $\left(\mathrm{QMt}_{\mathrm{s}}^{\prime} / \mathrm{t}_{1}\right) /[\mathrm{QMRes}(\mathrm{b})]$ \\
$\ldots$ & $\ldots$ & $\ldots$ \\
$\mathrm{H}_{\mathrm{o}}(2 . \mathrm{v})$ & $(1) ;\lfloor\mathrm{v}(\mathrm{u}-1)(\mathrm{r}-1)\rfloor$ & $\left(\mathrm{QMt}_{\mathrm{s}}^{\prime} / \mathrm{t}_{\mathrm{v}}\right) /[\mathrm{QMRes}(\mathrm{b})]$
\end{tabular}

onde,

$$
S Q t^{\prime} / t_{i}=\frac{1}{r}\left[\sum_{s=1}^{u} y_{i . s}^{2}-\frac{\left(\sum y_{i . s}\right)^{2}}{u}\right]
$$


Assim, a adoção desse procedimento modificaria apenas a "parte inferior" dos quadros de análise, que passaria a ser:

\begin{tabular}{cc}
\hline Causa da Variaçäo & Graus de liberdade \\
$t^{\prime} / t_{1}$ & $u-1$ \\
$t^{\prime} / t_{2}$ & $u-1$ \\
$\ldots$ & $\ldots$ \\
$t^{\prime} / t_{v}$ & $u-1$ \\
Residuo (b) & $v(u-1)(r-1)$ \\
\hline Total & urv-1
\end{tabular}

4.6.7 - Comparações mültiplas

Considerando a possibilidade de rejeição de ao menos uma das hipóteses de nulidade, formularam-se as regras para os testes de contrastes entre efeitos dos parâmetros envolvidos.

Naturalmente são utilizáveis quaisquer dos testes usuais para comparações mültiplas. Neste estudo, optou-se pelo teste Tukey, por sua versatilidade e grande aplicação na estatística expe rimental. Ademais, foram estudados os quatro casos clássicos, citados em PIMENTEL GOMES (1976). 


\subsubsection{1 - Comparação entre efeitos estimados de tra tamentos principais}

Considerou-se o critério do teste de Tukey, citadoem PIMENTEL GOMES (1976)

$$
\text { D.M.S. }=q \sqrt{(172) V(\gamma)}
$$

onde D.M.S. è a diferença mínima significativa, q é o valor da amplitude total estudentizada e $\hat{V}(\hat{\gamma})$ é a variância estimada do contraste estimado, $\hat{\gamma}$, entre efeitos médios.

$$
\text { Então, para um contraste } \hat{\gamma}_{i}\left(i, i^{\prime}\right)=\hat{m}_{i}-\hat{m}_{i}{ }^{\prime} \text {, resul- }
$$

tou, segundo $(\alpha .25)$

$$
\text { D.M.S. }=q \sqrt{\prime(k / \lambda u v) ~ Q ! R e s(a)} ; q_{\alpha, v, r v-r-a+1}
$$

que è um valor idêntico àqueles encontrados na literatura, para a diferença mínima significativa entre dois efeitos de tratamentos em ensaios com blocos incompletos balanceados.

\subsubsection{2 - Comparação entre efeitos estimados de tratamentos secundārios}

$$
\text { Para } \hat{\gamma}_{2}\left(s, s^{\prime}\right)=\hat{m}_{s}-\hat{m}_{s^{\prime}} \text {, com base em }(\alpha .33) \text { obteve- }
$$

se

$$
\text { D.M.S. }=q \sqrt{(17 \overline{r v}) \text { QMRes }}(\bar{b})^{-} ; q_{\alpha, u, v(u-1)(r-1)}
$$

que é um resultado idêntico àqueles encontrados na literatura para a diferença mínima significativa entre dois efeitos de tratamentos 
116.

secundärios em ensaios com parcelas subdivididas em blocos (complem tos) casualizados.

4.6.7.3 - Comparação entre efeitos estimados de tratamentos secundārios, dentro do i-ēsimo tratamento principal

Tomando-se o contraste

$$
\bar{\gamma}_{3, i}\left(s, s^{\prime}\right)=\hat{m}_{i s}-\hat{m}_{i s^{\prime}}
$$

obteve-se, segundo (a.39):

$$
\begin{gathered}
\text { D.M.S. }=q \sqrt{(1 / r)\left(1-\frac{1}{v}\right) \operatorname{QMRes}(b)} \\
q_{\alpha, u, v(u-1)(r-1)}
\end{gathered}
$$

Este valor não foi integralmente encontrado na literatura pesquisada. No entanto, CHAKRABARTI (1962) demonstra que a variância para contrastes do tipo de $\gamma_{3}$ para "Split-plot" em blocos (completos) casualizados, é a mesma obtida neste estudo em ( $\alpha .39$ ). Além disso, recomenda que para o teste de tais contrastes seja usado o quadrado médio do erro (b). 
117.

4.6.7.4 - Comparação entre efeitos estimados de tra tamentos principais, dentr do suësimo tratamento secundärio

Tomando-se o contraste

$$
\hat{\gamma}_{4, S}\left(i, i^{\prime}\right)=\hat{m}_{i s}-\hat{m}_{i^{\prime} s}
$$

resultou, segundo $(\alpha .41)$

$$
\begin{gathered}
\text { D.M.S. }=q \sqrt{(1 / r)\left(1-\frac{1}{u}\right) \operatorname{QMRes}(b)} \\
q_{\alpha, u, v(u-1)(r-1)}
\end{gathered}
$$

Este resultado foi contrastante com aquele encontrado por PIMENTEL GOMES (1976), que apresenta como variäncia para con trastes como $Y_{4}$, uma combinação linear entre os resíduos (a) e (b), quando o experimento em parcelas subdivididas é delineado em blocos (completos) casualizados. Em seu estudo, o autor apresenta um valor aproximado, $n^{\prime}$, derivado da fórmula de Satterthwaite, para os graus de liberdade relativos à combinação linear dos resíduos.

\section{7 - Testes sobre a Estrutura da Matriz de Covariāncias}

De acordo com GILL (1978) e LEAL (1979), dentre outros, a adequação do modelo proposto em (4.2), aos dados experimentais, está diretamente associada à realização das pressuposições so bre as matrizes de covariâncias entre tratamentos secundärios den- 
tro de cada tratamento principal, alēm das tradicionais hipóteses de normalidade, independência, aditividade, etc.

Assim, se não se verificam as hipöteses de homogenei dade e de uniformidade para as matrizes de covariâncias, esses auto res recomendam como alternativa ao modelo univariado, o uso das téc nicas da análise multivariada, mais especificamente a anālise de per fil, conforme amplamente abordado em LEAL (1979).

Com base em tais resultados, julgou-se recomendável que se apresentassem testes sobre a estrutura dessas matrizes que, segundo entendeu-se, devem ser parte integrante do processo de análise descrito neste estudo.

4.7.1 - Teste de homogeneidade para as matrizes de covariān cias

Testes dessa natureza têm por objetivo verificar se as $\underline{v}$ matrizes de covariâncias ${ }_{(u)}{ }^{Z_{i}}(u)$ podem ser representadas por uma única matriz $Z$, comum aos $\underline{v}$ tratamentos principais.

Dentre os mais usuais, optou-se, neste estudo, pelo critério de Box (1950), descrito por DANFORD et alii (1960) e GILL (1978), entre outros,

$$
\mathrm{H}_{0}: Z_{1}=Z_{2}=\ldots=Z_{\mathrm{v}}=Z \text {, }
$$

toma-se o seguinte critērio:

$$
g_{3}=(r-1)\left[v \text { \&n }|G|-\sum_{i=1}^{v} \text { \&n }\left|G_{i}\right|\right]
$$


onde, $\quad r=$ número de repetições por tratamento principal;

$$
\begin{aligned}
& v=\text { número de tratamentos principais; } \\
& G=\left(g_{s s},\right) \bar{e} \text {, sob a hipótese de nulidade, uma estimativa não } \\
& \text { tendenciosa da matriz de covariâncias comum } \mathbb{Z} \text {; } \\
& G_{i}=\left(g_{S s^{\prime}, i}\right) \bar{e} \text { a estimativa não tendenciosa para } \mathscr{Z}_{i}, i=1, \ldots, v \text {; } \\
& \text { a matriz de covariâncias entre tratamentos secundários, } \\
& \text { para o i-ésimo tratamento principal. }
\end{aligned}
$$

Ademais :

$$
\begin{gathered}
\ell n=\text { logarítmo neperiano, } \\
||=\text { determinante } \\
g_{s s^{\prime}}=\frac{1}{n-v} \sum_{i, j}\left(y_{i j k}-\bar{y}_{. j k}\right)\left(y_{i j k^{\prime}}-\bar{y}_{\cdot j k^{\prime}}\right) \\
g_{s s^{\prime}, i}=\frac{1}{r-1} \sum_{i}\left(y_{i j k}-\bar{y}_{. j k}\right)\left(y_{i j k^{\prime}}-\bar{y}_{\cdot j k^{\prime}}\right)
\end{gathered}
$$

Obs.: Aqui, G não se refere ao total geral.

Se os valores dos determinantes são próximos de zero, GILL (1978) recomenda que sejam usados logarítmos em base 10 e que se multiplique $g_{3}$ por 2,3026 .

A distribuição probabilística de $g_{3}$, não tem interes se prático para este estudo, no entanto, $\left(1-g_{1}\right) g_{3}$ tem distribuição aproximada de um $\chi^{2}$ com $f_{1}$ graus de liberdade, onde

$$
g_{1}=[(v+1) /(n-v)]\left(2 u^{2}+3 u-1\right) /[6(u+1)]
$$


e

$$
\mathrm{f}_{i}=[\mathrm{u}+\mathrm{u}(\mathrm{u}-1) / 2](\mathrm{v}-1)
$$

pois, dentro de cada tratamento existem u variâncias e u(u-1)/2 covariâncias diferentes, conforme visto no comentārio de $(\alpha .54)$, dentre outros.

$$
\text { Então, se }\left(1-g_{1}\right) g_{3}<\chi_{\alpha, f_{1}}^{2} \text {, aceita-se } H_{0} \text {, caso }
$$
contrário, conclui-se pela existência de ao menos um par $Z_{i} \neq Z_{i^{\prime}}$, para algum $i \neq i '$.

Por outro lado, essa aproximação é considerada adequada (MORRISON, 1976), se v<5, u<5 e rv > 20. Para GILL(1978), se $v \geq 5$ ou $u \geq 5$, ou ambos, uma melhor aproximação seria obtida através da distribuição $F$, com

$$
g_{3}\left[\left(1-g_{1}\right)-\left(f_{1} / f_{2}\right)\right] / f_{1} \cap F_{\alpha, f_{1}, f_{2}}
$$

onde, $f_{2}=\left(f_{1}+2\right) /\left(g_{2}-g_{1}^{2}\right)$

$$
g_{2}=\left(v^{2}+v+1\right)\left(u^{2}+u-2\right) /\left[6(n-v)^{2}\right]
$$

4.7.2 - Teste de uniformidade da matriz comum de covariāncias

Segundo LEAL (1979), a pressuposição da uniformidade da matriz comum de covariâncias pode ser testada através do critério de Wilks (1946), modificado por Box em 1949.

A hipótese de nulidade a ser testada ë 


$$
H_{0}: Z=Z *
$$

onde $Z *$ é uma matriz uniforme, definida de modo anālogo à matriz $W$ em $(\alpha .18)$.

$$
\begin{gathered}
0 \text { critério do teste é dado por } \\
\qquad g_{4}=\frac{|G|}{\left|G^{*}\right|}
\end{gathered}
$$

com

$$
G^{*}=\left(g_{s s^{\prime}}^{*}\right)=\left\{\begin{array}{l}
(1 / u) \sum_{s=1}^{u} g_{s s^{\prime}}, \text { se } s=s^{\prime} \\
1 /[u(u-1)] \sum_{s \neq s^{\prime}}^{u} g_{s s^{\prime}}
\end{array}\right.
$$

Determina-se, então, $g_{5}=-(n-v)$ ln $g_{4}$ e, sob a hipótese de nulidade $\left(1-g_{6}\right) g_{5}$ é aproximadamente distribuỉda segundo um $x^{2}$ com $f_{3}$ graus de liberdade, onde, segundo LEAL (1979),

$$
\begin{aligned}
& g_{6}=\frac{u(u+1)^{2}(2 u-3)}{6(n-v)(u-1)\left(u^{2}+u-4\right)} \\
& f_{s}=\left(u^{2}+u-4\right) / 2
\end{aligned}
$$

$$
\text { Desta feita, rejeita-se } H_{0} \text {, se }\left(1-g_{6}\right) g_{5}>x_{\alpha, f_{3}}^{2}
$$


122.

5. ILUSTRAÇAO DO METODO PROPOSTO

Conforme comentado em (3), julgou-se adequada a ilus tração dos resultados obtidos neste estudo. Assim, com base nos dados ali descritos (Tabela 1) foram efetuados os cálculos inerentes às fórmulas demonstradas e entendidas como mais importantes.

Inicialmente buscou-se informações sobre o cumprimento das exigências do modelo, através dos testes de homogeneidade e uniformidade das matrizes de covariâncias. A seguir foram ilustrados os resultados. 
5.1 - Testes Sobre a Estrutura das Matrizes de Covariāncias

5.1.1 - Teste de homogeneidade das matrizes de covariancias para todos os tratamentos principais

Denominando-se por $G_{i}, i=1, \ldots, 6$, as estimativas das matrizes de covariâncias $Z_{i}$ para os 6 tratamentos principais, obtiveram-se, com base em $(4.7 .1)$ :

$$
\begin{aligned}
& G_{1}=\left[\begin{array}{ccc}
7,54 & 5,71 & 5,13 \\
5,71 & 6,52 & 5,83 \\
5,13 & 5,83 & 6,18
\end{array}\right] \quad \begin{array}{l}
\therefore\left|G_{1}\right|=16,0061 \\
\text { e } \ln \left|G_{1}\right|=2,7730
\end{array} \\
& G_{2}=\left|\begin{array}{lll}
9,62 & 2.00 & 3,93 \\
2,00 & 5,14 & 3,84 \\
3,93 & 3,84 & 9,19
\end{array}\right| \quad \begin{array}{l}
\quad \therefore\left|G_{2}\right|=256,7814 \\
\text { e } \ln \left|G_{2}\right|=5,5482
\end{array} \\
& G_{3}=\left[\begin{array}{rrr}
9,41 & 0,59 & 1,87 \\
0,59 & 1,35 & -0,30 \\
1,87 & -0,30 & 9,30
\end{array}\right] \quad \begin{array}{l}
\quad \therefore\left|G_{3}\right|=108,6755 \\
\text { e } \ln \left|G_{3}\right|=4,6884
\end{array} \\
& G_{4}=\left[\begin{array}{lll}
7,90 & 1,99 & 2,40 \\
1,99 & 7,37 & 1,18 \\
2,40 & 1,18 & 5,71
\end{array}\right] \quad \begin{array}{l}
\quad \therefore\left|G_{4}\right|=267,6614 \\
\text { e } \ln \left|G_{4}\right|=5,5897
\end{array}
\end{aligned}
$$


124.

$$
\begin{aligned}
& G_{5}=\left[\begin{array}{rrr}
5,69 & 6,80 & 7,03 \\
6,80 & 20,69 & 10,50 \\
7,03 & 10,50 & 11,75
\end{array} \mid\right. \\
& \therefore\left|G_{5}\right|=194,0047 \\
& \text { e } \ln \left|G_{5}\right|=5,2679 \\
& G_{6}=\left[\begin{array}{lll}
9,37 & 0,75 & 6,51 \\
0,75 & 1,97 & 0,91 \\
6,51 & 0,91 & 7,73
\end{array}\right] \\
& \therefore\left|G_{6}\right|=55,9772 \\
& e \ln \left|G_{6}\right|=4,0249
\end{aligned}
$$

Desse modo, a estimativa $G$ para a matriz comum de co variâncias $Z$, ficou:

$$
G=\left[\begin{array}{lll}
8,255 & 2,973 & 4,478 \\
2,973 & 7,173 & 3,660 \\
4,478 & 3,660 & 8,310
\end{array}\right] \quad \begin{aligned}
& \text { e } \ln |G|=5|G|=261,6459 \\
&
\end{aligned}
$$

Então, para o teste de

$$
\begin{aligned}
& H_{0}: Z_{1}=Z_{2}=\ldots=Z_{6}=Z \\
& H_{a}: Z_{i} \neq Z_{i^{\prime}}, \text { para algum par } i, i^{\prime} \quad\left(i \neq i^{\prime}\right)
\end{aligned}
$$

foram obtidos, conforme (4.7.1).

$$
\begin{aligned}
& g_{3}=9\left(6 \ln |G|-\sum_{i=1}^{6} \ln \left|G_{i}\right|\right)=49,5891 \\
& g_{1}=\frac{7}{54} \cdot \frac{26}{24}=0,1404 \\
& \therefore\left(1-g_{1}\right) g_{3}=\chi_{o b s}^{2}=42,62
\end{aligned}
$$




$$
\begin{gathered}
f_{1}=\left(3+\frac{3.2}{2}\right) 5=30 \mathrm{~g} \cdot 1 . \\
\text { Assim, como } x_{\text {obs }}^{2}=42,62<x_{\text {tab }}^{2}=x_{5 \%, 30}^{2}=43,77 \text {, não }
\end{gathered}
$$

houveram evidências que indicassem a rejeição de H. $_{0}$

Adotou-se, então, a matriz comum de covariâncias $\Sigma$, estimada pela matriz G, para representar as covariâncias entre tratamentos secundārios, dentro de cada tratamento principal.

\subsection{2 - Teste de uniformidade da matriz comum de covariân- cias}

Reportando-se a (4.7.2), determinou-se a estimativa $G^{*}$, da matriz uniforme $Z^{*}$ :

$$
G^{*}=\left[\begin{array}{lll}
7,9127 & 3,7037 & 3,7037 \\
3,7037 & 7,9127 & 3,7037 \\
3,7037 & 3,7037 & 7,9127
\end{array}\right] \quad \therefore\left|G^{*}\right|=271,4060
$$

Assim, para o teste de

$$
\begin{aligned}
& \mathrm{H}_{0}: Z=Z^{*} \\
& \mathrm{H}_{\mathrm{a}}: Z \neq Z^{*}
\end{aligned}
$$

foram obtidos:

$$
\begin{aligned}
& g_{4}=\frac{|G|}{|G *|}=0,9640 \quad \therefore \quad \ln g_{4}=-0,0367 \\
& g_{5}=-(n-v) \text { ln } g_{4}=1,9818 \\
& g_{6}=\frac{144}{5184}=0,0278
\end{aligned}
$$




$$
\begin{gathered}
\therefore\left(1-g_{t}\right) g_{5}=x_{o b s}^{2}=1,9267 \\
\mathrm{f}=\frac{8}{2}=4.1 . \\
\text { Entao, como } x_{\text {obs }} \cong 1,93<x_{t a b}^{2}=x_{5 \%, 4}^{2}=9,49, \text { não }
\end{gathered}
$$
houveram evidências que induzissem à rejeição de $H_{0}$.

Adotuu-se, entào, a estimativa $G^{*}$, da matriz uniforme de covariâncias $z^{*}$, para todos os tratamentos.

\section{2 - Equaçōes Noma is}

Verificadas as exigências do modelo matemático, determinou-se o sistema de equações normais, conforme $(\alpha .1)$

$$
\left[\begin{array}{ccccc}
\mathrm{n} & \mathrm{x} & \mathrm{y} & \mathrm{z} & \mathrm{w} \\
\mathrm{x}^{\prime} & \mathrm{R} & \mathrm{N} & \mathrm{P} & \mathrm{S} \\
\mathrm{y}^{\prime} & \mathrm{N}^{\prime} & \mathrm{A} & \mathrm{K} & \mathrm{V} \\
\mathrm{z}^{\prime} & \mathrm{P}^{\prime} & \mathrm{K}^{\prime} & \mathrm{U} & \mathrm{H} \\
\mathrm{w}^{\prime} & \mathrm{S}^{\prime} & \mathrm{V}^{\prime} & \mathrm{H}^{\prime} & \mathrm{L}
\end{array}\right]\left[\begin{array}{c}
\hat{\mathrm{m}} \\
\hat{\mathrm{T}} \\
\hat{\mathrm{B}} \\
\hat{\tau} \\
\hat{\delta}
\end{array}\right]=\left[\begin{array}{c}
\mathrm{G} \\
\mathrm{T} \\
\mathrm{B} \\
\mathrm{T} \\
\Delta
\end{array}\right]
$$

onde, para $i=1, \ldots, v=6$ tratamentos principais,

$j=1, \ldots, a=15$ blocos,

$\mathrm{s}=1, \ldots, \mathrm{u}=3$ tratamentos secundārios,

$r=10$ repetições por tratamento principal,

$k=4$ parcelas por bloco, 
$\mathrm{n}=u r v=180$

$$
\begin{aligned}
& x=30 \cdot \frac{E}{(1)^{(6)}} \\
& y=12 . \\
& \text { (1) (15) } \\
& z=60 . \quad{ }_{\text {(1) }} \text { (3) } \\
& w=10 \cdot \frac{E}{(1)(18)} \\
& R=30 \cdot I_{(6)} \\
& P=10 \cdot \frac{E}{(6)(3)} \\
& A=12 \cdot I_{(15)} \\
& K=4 \cdot \frac{E}{(15)(3)} \\
& U=60 \cdot I_{(3)} \\
& L=10 \cdot \frac{E}{(18)(18)} \\
& \mathrm{H}=\left[\begin{array}{ll:l}
10 & \left.\mathrm{I}_{(3)}: 10 \cdot \mathrm{I}_{(3)}: \ldots: 10 \cdot \mathrm{I}_{(3)}\right]
\end{array}\right.
\end{aligned}
$$

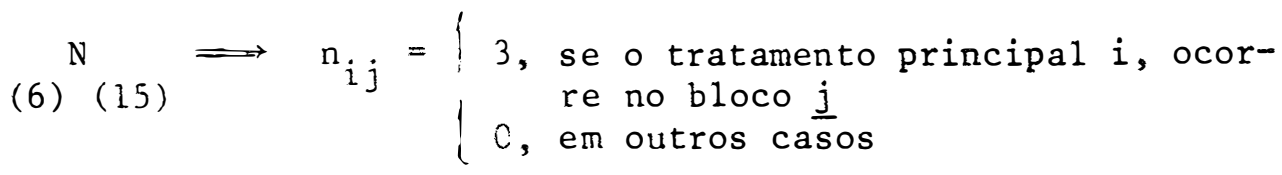

$$
\begin{aligned}
& S=\left[\begin{array}{cccccccccc}
10 & 10 & 10 & 0 & 0 & 0 & \ldots & 0 & 0 & 0 \\
0 & 0 & 0 & 10 & 10 & 10 & \ldots & 0 & 0 & 0 \\
\ldots & \ldots & \ldots & \ldots & \ldots & \ldots & \ldots & \ldots & \ldots & \ldots \\
0 & 0 & 0 & 0 & 0 & 0 & \ldots & 10 & 10 & 10
\end{array}\right] \\
& \text { (6) }
\end{aligned}
$$




$$
\begin{aligned}
& \begin{array}{lllllllllllll}
11 & 12 & 13 & 21 & 22 & 23 & 31 & 32 & 33 & \ldots & 61 & 62 & 63
\end{array} \\
& \left.\begin{array}{lllllllllllllll}
1 & 1 & 1 & 1 & 1 & 1 & 1 & 1 & 1 & 1 & \ldots & 0 & 0 & 0
\end{array}\right]
\end{aligned}
$$

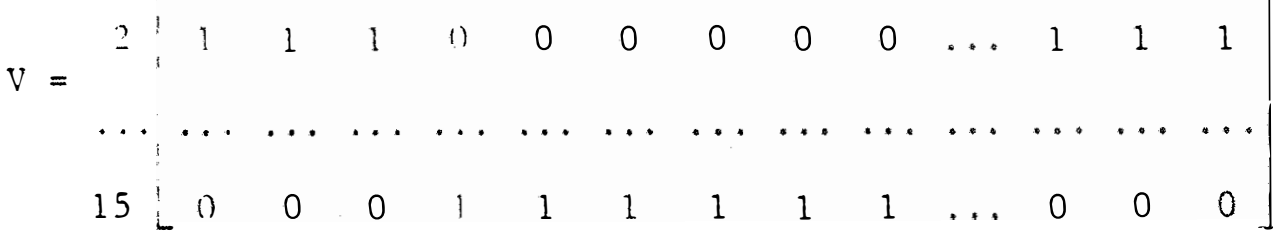

$$
\begin{aligned}
& \hat{T}=\left[\begin{array}{c}
\hat{t}_{1} \\
\hat{t}_{2} \\
\cdots \\
\hat{t}_{6}
\end{array}\right] \\
& \widehat{B}=\left[\begin{array}{c}
b_{1} \\
b_{2} \\
\cdots \\
\sigma_{15}
\end{array}\right] \\
& \hat{\tau}^{*}=\left[\begin{array}{c}
\hat{t}_{1}^{\prime} \\
\hat{E}_{1}^{\prime} \\
\hat{E}_{3}^{\prime}
\end{array}\right] \\
& \hat{\delta}=\left[\begin{array}{c}
t \hat{t}_{11}^{\prime} \\
\hat{t} \hat{t}_{12}^{\prime} \\
\hat{t t_{13}^{\prime}} \\
\cdots \\
\cdots \\
\hat{t} \hat{t}_{61}^{\prime} \\
t t_{62}^{\prime} \\
\hat{t} \hat{t}_{63}^{\prime}
\end{array}\right] \\
& G=3190,3
\end{aligned}
$$


129.

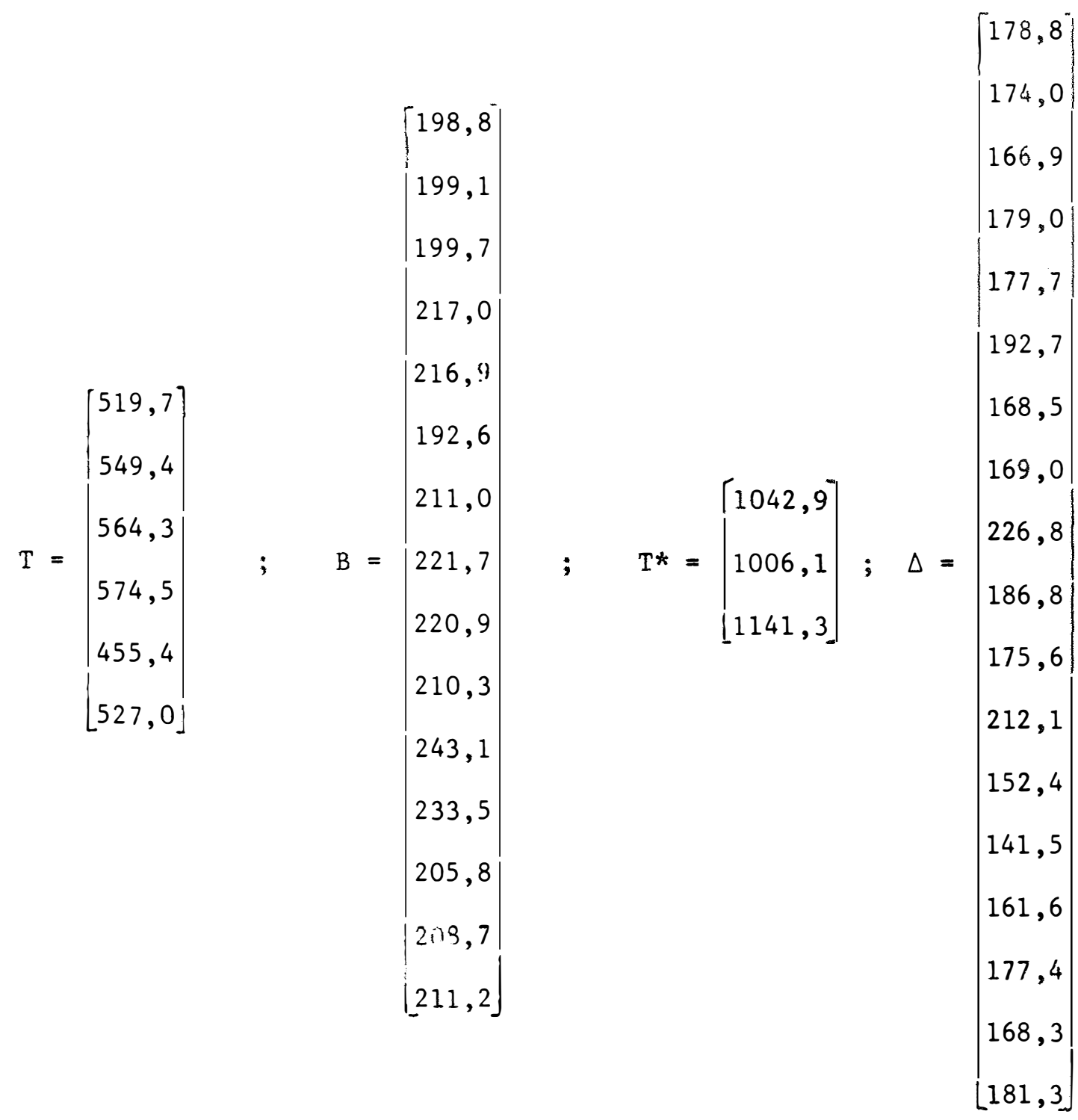




\section{3 - Solução do Sistema}

5.3.1 - Efeitos estimados de tratamentos principais

$$
\text { Reportando-se a }(\alpha .12) \text {, para a solução } \hat{\tau}=M^{-1} Q \text {, ob- }
$$

teve-se

$$
M^{-1}=\frac{1}{27} I_{(6)} \quad Q=\left[\begin{array}{r}
-13,400 \\
18,125 \\
29,975 \\
46,075 \\
-74,900 \\
-5,825
\end{array}\right] ; \sum_{i=1}^{6} Q_{i}=0
$$

$$
\therefore \quad \hat{\tau}=\left[\begin{array}{r}
-0,4965 \\
0,6711 \\
1,1101 \\
1,7063 \\
-2,7743 \\
-0,2159
\end{array}\right] ; \sum_{i=1}^{6} \hat{t}_{i}=0
$$

5.3.2 - Efeitos estimados de tratamentos secundārios

De acordo com $(\alpha .4)$, obteve-se

$$
\hat{\mathrm{m}}=17,7239 \mathrm{~h}
$$

e, segundo $(\alpha .13)$ ou $(\alpha .14)$, resultou: 


$$
\hat{\tau} *=\left[\begin{array}{c}
-0,3422 \\
-0,9555 \\
1,2977
\end{array}\right] ; \quad \sum_{s=1}^{3} \hat{t}_{s}^{\prime}=0 .
$$

\section{3 .3 - tfeitos estimados dos pares $\left(t t^{\prime}\right)_{\text {is }}$}

Segundo (a.16), foram obtidas as estimativas dos efei tos de tratamentos principais de modo usual, isto é, sem considerar-se o ajuste para efeitos estimados de blocos, obtendo-se:

$$
\tau=\left[\begin{array}{r}
-0,4006 \\
0,5894 \\
1,0861 \\
1,4261 \\
-2,5439 \\
-0,1572
\end{array}\right] ; \quad \sum_{i=1}^{6} \tilde{t}_{i}=0
$$

Assim, através das estimativas $\tilde{t}_{i}$, determinaram-se os componentes do vetor $\hat{\delta}$ :

$$
\begin{aligned}
& \hat{t t_{11}}=0,8989 \\
& \hat{E}_{j_{2}}^{\prime}=7,0321 \\
& t \hat{t}_{13}^{\prime}=-1,9310 \\
& \hat{t}_{21}^{\prime}=-0,0711 \\
& \hat{t}_{22}^{\prime}=0,4122 \\
& \hat{t t_{23}^{\prime}}=-0,3411 \\
& \hat{t}_{31}^{\prime}=-1,6178 \\
& \hat{t}_{32}^{\prime}=-0,9545 \\
& \hat{E t_{3}} \hat{3}_{3}=2,5723 \\
& \hat{t t_{41}^{\prime}}=-0,1278 \\
& \hat{E t_{42}^{\prime}}=-0,6345 \\
& t t_{43}^{\prime}=0,7623 \\
& \hat{t t_{51}^{\prime}}=0,4022 \\
& t \hat{t}_{5_{2}}^{\prime}=-0,0745 \\
& t t_{53}^{\prime}=-0,3177 \\
& \overline{t E}_{b 1}^{\prime}=0,5155 \\
& t \hat{t}_{62}^{\prime}=Q, 2188 \\
& t t_{63}^{\prime}=-0,7343
\end{aligned}
$$


onde observou-se

$$
\begin{aligned}
& \sum_{i}\left(t t^{\prime}\right)_{\text {is }}=0, \forall s \\
& \sum_{s}\left(t t^{\prime}\right)_{\text {is }}=0, \forall i \\
& \sum_{i, s}\left(t t^{\prime}\right)_{\text {is }}=0
\end{aligned}
$$

5.4 - Anälise de Variāncia

5.4.1 - Obtenção das somas de quadrados

Reportando-se a (4.6), foram determinadas:

$$
\begin{aligned}
& \operatorname{SQt}(\operatorname{aj} .)=\hat{\tau}^{\prime} Q=339,7625 \\
& \operatorname{SQb}=B^{\prime} A^{-1} B-C_{0}=\frac{1}{12} \sum_{j=1}^{15} B_{j}^{2}-C_{0}
\end{aligned}
$$

onde $C_{0}=\frac{(3190,3)^{2}}{180}=56544,5220$

$$
\begin{aligned}
\mathrm{SQb} & =213,9050 \\
\text { SQGrupos } & =\frac{1}{36} \sum_{\mathrm{g}=1}^{5} \mathrm{G}_{\mathrm{g}}^{2}-\mathrm{C}_{\mathrm{o}}=126,3940 \\
\mathrm{SQb} / \mathrm{Gru} & =213,9050-126,3940=87,5110 \\
\mathrm{SQParc} & =\frac{1}{3} \sum_{i j} \mathrm{y}_{i j}^{2} .-\mathrm{C}_{\mathrm{o}}=1133,9340
\end{aligned}
$$


$\operatorname{SQReS}(a)=580,2665$

$$
S Q t^{\prime}=T^{*} U^{-1} T *-C_{0}=\frac{1}{60} \sum_{S} T_{S}^{* 1}-C_{0}
$$

$\therefore \quad$ SQt $^{\prime}=162,8630$

$$
\begin{aligned}
\mathrm{SQ} \delta & =\Delta^{\prime} \mathrm{L}^{-1} \Delta-\mathrm{C}_{0}-\mathrm{SQt}-\mathrm{SQ} \mathrm{t}^{\prime} \\
& =\frac{1}{10} \sum_{\mathrm{i}, \mathrm{S}}\left(\mathrm{TT}^{\prime}\right)_{i \mathrm{~S}}^{2}-\mathrm{C}_{0}-\mathrm{SQt}-\mathrm{SQt^{ \prime }} \\
& =181,6670 \\
\mathrm{SQT} & =\mathrm{Y}^{\prime} \mathrm{Y}-\mathrm{C}_{\mathrm{O}}=1937,4400
\end{aligned}
$$

$\operatorname{SQReS}(b)=458,9760$

5.4 .2 - Testes de hipōteses e quadros de anāilise

Obteve-se, então, o seguinte quadro para a análise de variância:

\begin{tabular}{lccrc}
\hline $\begin{array}{l}\text { Causa da } \\
\text { Variação }\end{array}$ & $\begin{array}{c}\text { Graus de } \\
\text { liberdade }\end{array}$ & $\begin{array}{c}\text { Somas de } \\
\text { quadrados }\end{array}$ & $\begin{array}{c}\text { Quadrados } \\
\text { médios }\end{array}$ & F(observado) \\
\hline Grupos & 4 & 126,3940 & 31,5985 & 2,25 \\
BL/Gru & 10 & 87,5110 & 8,7511 & 0,62 \\
Trat. (aj.) : $t$ & 5 & 339,7625 & 67,9525 & $4,83 * *$ \\
Residuo (a) & 40 & 580,2665 & 14,0667 & \\
\hline Parcelas & $(59)$ & $(1133,9340)$ & & \\
\hline Trat.: t & 2 & 162,8630 & 81,4315 & $19,16 * *$ \\
tx t & 10 & 181,6670 & 18,1667 & $4,27 * \star$ \\
Residuo (b) & 108 & $458 ; 9760$ & 4,2498 & \\
\hline Total & 179 & 1937,4400 & & \\
\hline
\end{tabular}


que forneceu evidências para a rejeição de $\mathrm{H}_{0}(1), \mathrm{H}_{\mathrm{O}}(2)$ e $\mathrm{H}_{\mathrm{O}}(3) ;$ ao nivel de $1 \%$ de probabilidade. Concluiu-se, então, pela existência de efeito diferencial significativo entre tratamentos principais, entre tratamentos secundários e efeito significativo da interação, respectivamente.

De acordo com (4.6.6), a rejeição de $H_{0}(3)$ motivou, segundo PIMENTEL GOMES (1976), a aplicação dos testes para $\mathrm{H}_{0}(2 . i)$, resultando:

\begin{tabular}{lcrrr}
\hline $\begin{array}{l}\text { Causa da } \\
\text { Variação }\end{array}$ & $\begin{array}{c}\text { Graus de } \\
\text { liberdade }\end{array}$ & $\begin{array}{c}\text { Somas de } \\
\text { quadrados }\end{array}$ & $\begin{array}{c}\text { Quadrados } \\
\text { médios }\end{array}$ & F(observado) \\
\hline Grupos & 4 & 126,3940 & 31,5985 & 2,25 \\
Bl/Gru & 10 & 87,5110 & 8,7511 & 0,62 \\
Trat. (aj.) : $t$ & 5 & 339,7625 & 67,9525 & $4,83 * *$ \\
Residuo (a) & 40 & 580,2665 & 14,0667 & \\
\hline Parcelas & $(59)$ & $(1133,9340)$ & & \\
\hline$t^{\prime} / t_{1}$ & 2 & 7,1687 & 3,5843 & 0,84 \\
$t^{\prime} / t_{2}$ & 2 & 13,8130 & 6,9065 & 1,62 \\
$t^{\prime} / t_{3}$ & 2 & 224,6660 & 112,3330 & $26,43 * *$ \\
$t^{\prime} / t_{4}$ & 2 & 69,9260 & 34,9630 & $8,23 * *$ \\
$t^{\prime} / t_{5}$ & 2 & 20,0540 & 10,0270 & 2,36 \\
$t^{\prime} / t_{6}$ & 2 & 8,9007 & 4,4503 & 1,05 \\
Residuo (b) & 108 & 458,9760 & 4,2498 & \\
\hline Total & 179 & 1937,4400 & & \\
\hline
\end{tabular}

rejeitando-se, desse modo, as hipöteses $H_{0}(2.3)$ e $H_{0}(2.4)$, ao nivel de $1 \%$ de probabilidade. 
135.

5.5 - Comparaçoes Múltiplas

Segundo (4.6.7), dadas as rejeiçöes das três hipóteses básicas: $\mathrm{H}_{\mathrm{O}}(1), \mathrm{H}_{\mathrm{O}}(2)$ e $\mathrm{H}_{\mathrm{O}}(3)$, foram efetuadas as comparações múltiplas, como a seguir.

5.5.? - Entre médias de efeitos estimados de tratamentos principais

Foram tomados os contrastes $\hat{\gamma}_{1}\left(i, i^{\prime}\right)=\hat{m}_{i}-\hat{m}_{i}$, e de terminadas, segundo (x.86)

$$
\begin{aligned}
& \text { D.M.S. }(5 \%)=(4,23)[(4)(14,0667) /(108)]^{1 / 2}=3,05 \\
& \text { D.M.S. }(1 \%)=(5,11)[(4)(14,0667) /(108)]^{1 / 2}=3,69
\end{aligned}
$$

resultando:

$$
\begin{aligned}
& \hat{y}(1,2)=0,99 \\
& \hat{r}(1,3)=1,49 \quad \hat{v}_{1}(2,3)=0,50 \\
& \therefore(1,4)=1,83 \quad \hat{\gamma}_{1}(2,4)=0,84 \quad \hat{\gamma}_{1}(3,4)=0,34 \\
& \therefore(1,5)=2,14 \hat{\gamma}_{1}(2,5)=3,13 * \hat{\gamma}_{1}(3,5)=3,63 * \hat{\gamma}_{1}(4,5)=3,97 * * \\
& (1,6)=0,25 \quad \hat{Y}_{1}(2,6)=0,74 \quad \hat{Y}_{1}(3,6)=1,24 \quad \hat{Y}_{1}(4,6)=1,58 \quad \hat{Y}_{1}(5,6)=2,39 \\
& 5.5 .2 \text { - Entre médias de efeitos estimados de tratamentos } \\
& \text { secundários } \\
& \text { Para os contrastes } \hat{\gamma}_{2}\left(s, s^{\prime}\right)=\hat{m}_{s}=\hat{m}_{s}^{\prime} \text {, foram obti- } \\
& \text { das, segundo }(\alpha .87) \text { : }
\end{aligned}
$$


136.

$$
\begin{aligned}
& \text { D.M.S. }(5 \%)=3,37[(4,2498) /(60)]^{1 / 2}=0,90 \\
& \text { D.M.S. }(1 \%)=4,22[(4,2498) /(60)]^{1 / 2}=1,12
\end{aligned}
$$

resultando

$$
\begin{aligned}
& \hat{Y}_{:(1,2)}=0,61 \\
& \hat{\gamma}_{i}(1,3)=1,64 * * \quad \hat{\gamma}_{2}(2,3)=2,25 * *
\end{aligned}
$$

5.5 .3 - Entre médias de efeitos estimados de tratamentos secundários, dentro do i-ésimo tratamento principa 1

$$
\text { Para os contrastes do tipo } \hat{\gamma}_{3, i}\left(s, s^{\prime}\right)=\hat{m}_{i s}-\hat{m}_{i s} \text { ' }
$$

foram obtidas, segundo ( $(.88)$ :

$$
\begin{aligned}
& \text { D.M.S. }(5 \%)=3,37\left[(1 / 10)\left(1-\frac{1}{6}\right)(4,2498)\right]^{1 / 2}=2,00 \\
& \text { D.M.S. }(1 \%)=4,22\left[(1 / 10)\left(1-\frac{1}{6}\right)(4,2498)\right]^{1 / 2}=2,51
\end{aligned}
$$

resultando:

$$
\begin{array}{lll}
\hat{\gamma}_{3,1}(1,2)=0,38 & \hat{\gamma}_{3,1}(1,3)=1,09 & \hat{\gamma}_{3,1}(2,3)=0,71 \\
\hat{\gamma}_{3,2}(1,2)=0,13 & \hat{\gamma}_{3,2}(1,3)=1,37 & \hat{\gamma}_{3,2}(2,3)=1,50 \\
\hat{\gamma}_{3,3}(1,2)=0,05 & \hat{\gamma}_{3,3}(1,3)=5,75 * * & \hat{\gamma}_{3,3}(2,3)=5,78 * * \\
\hat{\gamma}_{3,4}(1,2)=1,12 * * & \hat{\gamma}_{3,4}(1, \dot{i})=2,53 * * & \hat{\gamma}_{3,4}(2,3)=3,65 * *
\end{array}
$$




$$
\begin{aligned}
& i=5 \\
& \hat{\gamma}_{3,5}(1,2)=1,09 * \quad \hat{\gamma}_{3,5}(1,3)=0,91 * \quad \hat{\gamma}_{3,5}(2,3)=2,00 * * \\
& i=6 \\
& \hat{\gamma}_{3,6}(1,2)=0,91 * \quad \hat{\gamma}_{3,6}(1,3)=0,39 \quad \hat{\gamma}_{3,6}(2,3)=1,30 *
\end{aligned}
$$

5.5.4 - Entre médias de efeitos estimados de tratamentos principais dentro do s-ésimo tratamento secundārio

Para os contrastes $\hat{\gamma}_{4, s}\left(i, i^{\prime}\right)=\hat{m}_{i s}-\hat{m}_{i^{\prime} s}$, foram ob tidas, segundo $(\alpha .89)$ :

$$
\begin{aligned}
& \text { D.M.S. } \left.(5 \%)=4,11\left[(1 / 10)\left(1-\frac{1}{3}\right) 4,2498\right)\right]^{1 / 2}=2,19 \\
& \text { D.M.S. } \left.(1 \%)=4,90\left[(1 / 10)\left(1-\frac{1}{3}\right) 4,2498\right)\right]^{1 / 2}=2,61
\end{aligned}
$$

resultando:

$$
s=1
$$

$$
\begin{aligned}
& \hat{Y}_{4,1}(1,2)=0,02 \\
& \hat{r}_{1,1}^{4}(1,3)=1,03 \quad \hat{y}_{4}(2,3)=1,05 \\
& \hat{r}^{* 11}(1,4)=0,80 \quad \hat{r}^{* \prime \prime}(2,4)=0,78 \quad \hat{\gamma}_{4,1}(3,4)=1,83
\end{aligned}
$$

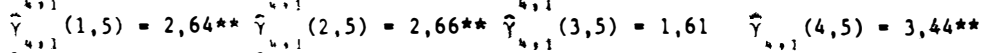

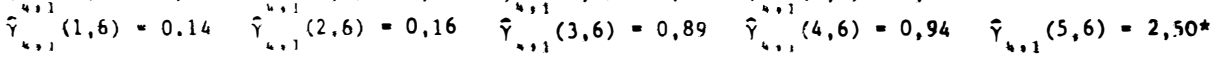

$$
\begin{aligned}
& s=2 \\
& \hat{Y}_{1,2}(1,2)=0,37 \\
& \hat{r}_{4,2}^{\prime \prime 2}(1,3)=0,50 \quad \hat{r}_{4, .}(2,3)=0,87 \\
& \hat{\gamma}_{6,2}(1,4)=0,16 \quad \hat{\gamma}_{1,2}^{4,2}(2,4)=0,21 \quad \hat{\gamma}_{4.2}(3,4)=0,66
\end{aligned}
$$

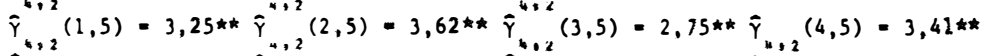

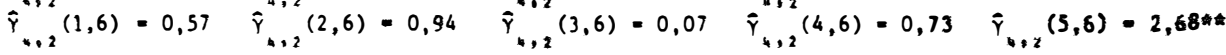

$$
\begin{aligned}
& s=3 \\
& \hat{Y}_{1,3}(1,2) \cdot 2,58 * \\
& \hat{Y}_{\ldots, 3}(1,3)=5,99 * k \hat{Y}_{4,3}(2,3)=3,41 * * \\
& \hat{Y}^{* 1,3}(1,4)=4,52 * \hat{Y}^{6,3}(2,4)=1,94 \quad \hat{Y}_{1},(3,4)=1,47
\end{aligned}
$$

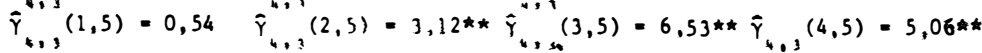

$$
\begin{aligned}
& \hat{\gamma}_{\ldots, 3}(1,6)=1,44 \quad \hat{\gamma}_{4,3}(2,6)=1,14 \quad \hat{\gamma}_{\ldots,}(3,6)=4,55 * \hat{\gamma}_{\ldots,}(4,6)=3,08 * \star \hat{\gamma}_{\ldots 3}(5,6)=1,98
\end{aligned}
$$


138.

6. CONCLUSOEES

Considerando-se os objetivos deste estudo, citados em (1.2) julgou-se desejável que as conclusões se dessem em termos de comparações dos resultados aqui obtidos, com aqueles encontrados na literatura para ensaios em blocos incompletos balanceados e/ou ensaios com parcelas subdivididas em blocos (completos) casualizados.

Sob esse aspecto, de modo geral concluiu-se

6.1 - Em Relação ao Modelo Matemático

O modelo matemático tradicionalmente utilizado para experimentos com parcelas subdivididas em blocos (completos) casualizados, foi adotado para o delineamento proposto neste estudo, sem apresentar desvantagens aparentes, mormente quando se desejou testar as hipóteses básicas de nulidade sobre os tratamentos princi- 
pais, os tratamentos secundários e a interação t $t^{\prime}$.

6.2 - Em Relação ao Sistema de Equações Norma is

O sistema de equações normais, obtido através do método dos mínimos quadrados:

i) portou-se de modo análogo aos ensaios em blocos incompletos balanceados, quando das estimativas para efeitos de tratamentos principais ajustados para efeitos de blocos;

ii) portou-se como nos ensaios com parcelas subdivididas em blo cos (completos) casualizados, quando das estimativas para efeitos de tratamentos secundārios e interação.

6.3 - Em Relação às Variāncias das Funções Lineares Estimāveis

O estudo das variâncias das funções lineares estimáveis, atravēs das matrizes de dispersão determinadas neste estudo, para os parâmetros básicos, realçou as conclusões de 6.2 , isto é:

i) a variância obtida para contrastes entre médias de tratamentos principais, mostrou-se semelhante àquelas obtidas para contrastes entre médias de tratamentos, nos ensaios em blocos incompletos balanceados;

ii) as variāncias obtidas para contrastes entre médias de tratamentos secundārios, entre médias de tratamentos secundā- 
rios "dentro" de um mesmo tratamento principal e entre mëdias de tratamentos principais "dentro" de um mesmo tratamento secundārio, foram semelhantes àquelas encontradas na bibliografia dos ensaios com parcelas subdivididas em blocos (completos) casualizados.

\section{4 - Em Relação à Anālise de Variāncia}

\subsection{1 - Quadro da anālise}

A anālise de variância para ensaios como o aqui proposto, mostrou-se divisivel em duas anālises independentes:

i) a primeira envolvendo blocos, tratamentos principais e o re sỉduo (a). Nesta anālise, que é semelhante às análises de ensaios em blocos incompletos balanceados, deve ser conside rado o tipo de BIB envolvido;

ii) a segunda, envolvendo tratamentos secundários, a interação tt' e o resíduo (b), que é semelhante às suas correspondentes em ensaios com parcelas subdivididas em blocos (completos) casualizados, inclusive na determinação da SQtxt'.

\section{4 .2 - Testes de hipōteses}

Os testes das três hipöteses básicas das análises de ensaios em parcelas subdivididas, portaram-se como de modo usual, no tocante aos resíduos adequados, inclusive para os efeitos de trata- 
mentos principais ajustados para efeitos de blocos.

Os testes das hipóteses adicionais, com respeito às repetições ou aos grupos, induzidas pelo uso do delineamento incompleto, portaram-se como nos ensaios em blocos incompletos balanceados.

\section{5 - Em Relação às Comparações Mūttiplas}

o uso do teste de Tukey, com base nas variâncias dos contrastes citados em (6.3), permitiu concluir pela coerência com os resultados anteriores: o procedimento usado para as comparações múltiplas entre tratamentos principais mostrou-se análogo ao procedimento usual dos ensaios em blocos incompletos balanceados, enquan to que as demais comparações seguiram os princípios dos ensaios com parcelas subdivididas em blocos (completos) casualizados.

Ademais, face aos resultados obtidos, concluiu-se que se $a=r$, onde a representa o número de blocos e $\underline{\underline{r}}$ o número de repeti ções, recai-se sobre a teoria dos ensaios com parcelas subdivididas em blocos (completos) casualizados, que ficaram caracterizados como casos particulares do delineamento proposto. 
BRADLEY, R.A.; R.E. WALPOLE e C.Y. KRAMER, 1960. Intra and Inter Block Analysis for Factorials in Incomplete Block Designs. Biometrics, Raleigh, 16: 566-581.

CALZADA BENZA, J., 1970. Métodos Estadísticos para la Investigación. 3. ed., Editorial Jurídica, Lima, Peru, 643 pp.

CHAKRABARTI, M.C., 1962. Mathematics of Design and Analysis of Experiments. 1 a ed. Asia Publishing House, Londres, 120 pp.

COCHRAN, W.G., 1951. Testing a Linear Relation Among Variances. Biometrics, Raleigh, 7: 17-32.

COCHRAN, W.G. e G.M. COX, 1976. Diseños Experimentales. 3. ed. Trillas, México, 661 pp.

DANFORD, M.B.; H.M. HUGHES e R.C. MCNEE, 1960. On the Analys is of Repeated-Measurements Experiments. Biometrics, Raleigh, 16: 547-565 . 
DEY, A., 1975. A Note on Balanced Designs. Sankyã, Calcutá, 37: 461-462.

DINIZ, U.D., 1980. Análise de Experimentos com Parcelas Medidas Su cessivamente no Tempo. ESALQ/USP, Piracicaba, 104 pp. (Tese de Doutoramento).

FEDERER, W.T., 1955. Experimenta1 Design. 1. ed., McMillan Co., Nova York, 544 pp.

GILL, L.G., 1978. Design and Analysis of Experiments in the Animal. and Medical Sciences. The Iowa State University Press. 1. ed. Ames, 3 vol. $882 \mathrm{pp}$.

GILL, J.L. e H.D. HAFS, 1971. Analysis of Repeated Measurements of Animals. J.An. Sci., Albany, 33: 331-336.

GOULDEN, C.H., 1939. Methods of Statistical Analysis. 1. ed. Wiley, Nova York, 277 pp.

GRAYBILL, F.A., 1976. Theory and Application of the Linear Model. 1. ed., Duxbury Press, Massachusetts, 703 pp.

HARTER, H.L., 1961. On the Analysis of Split-Plot Experiments. Biometrics, Raleigh, 18: 144-149.

HICKS, C.R., 1973. Fundamental Concepts in the Design of Experi-. ments. $2^{\text {a }}$ ed. Holt, Rinehart an Winston Co., Nova York, 349 pp.

KEMPTHORNE, O., 1950. The Design and Analysis of Experiments. 1. ed. Wiley, Nova York, 473 pp. 
LEAL, M.L.S., 1979. Análise de Dados Experimentais com Medidas Repetidas. Universidade Federal de Brasilia, Brasilia, 99 pp. (Dis sertação de Mestrado).

LEONARD, W.H. e A.G. CLARK, 1939. Field Plot Technique. Burgess. Minneapolis, 288 pp.

LITTLE, T.M. e F.J. HILlS, 1972. Statistical Methods in Agricultural Research. $1{ }^{a}$ ed., Univ. of California Press, Davis, 242 pp.

LI, S.H. e J.H. KLOTZ, 1978. Components of Variance Estimation for the Split-Plot Design. J.A.S.A., 73: 147-152.

MORRISON, D.F., 1976. Multivariate Statistical Methods. 2\% ed. McGraw Hill, Nova York, 415 pp.

PIMENTEL GOMES, F., 1967. The Solution of Normal Equations of Experiments in Incomplete Blocks. Ciência e Cultura, São Paulo, 20: $733-746$.

PIMENTEL GOMES, F., 1976. Curso de Estatistica Experimental. 6?. ed. Nobel, Piracicaba, 430 pp.

PURI, P.D., 1975. A Note on Efficiency Balanced Design. Sankyãa, Calcutá, 37: 457-460.

RAGHAVARAO, D., 1971. Combinatorial Problems in Design of Experiments. 1. ed. Willey, Nova York, 386 pp.

RAO, R., 1967. Least Squares Theory Using An Estimated Dispersion Matrix and Its Application to Measurement of Signals. Fifth Berk. Symp. V. 1 .

REES, D.H., 1969. The Analysis of Variance of Some Non Ortogonal Designs With Split-Plots. Biometrika, Londres, 56: 43-54. 
ROBINSON, J., 1966. Balanced Incomplete Block Designs with Double Grouping of Blocks Into Replications. Biometrics, Raleigh, 22: $368-373$.

ROBINSON, J., 1967. Incomplete Split Plot Designs. Biometrics, Raleigh, 23: 793-802.

SCHEFFE, H., 1959. The Analys is of Variance. 1. ed., Willey, Nova York, $477 \mathrm{pp}$.

SEARLE, S.R., 1971. Linear Models. 1. ed., Willey, Nova York, 531 pp.

SNEDECOR, G.W. e W.G. COCHRAN, 1969. Statistical Methods. 6. ed. The Iowa State University Press, Ames, 593 pp.

STEEL, R.G.D. e J.H. TORRIE, 1960. Principles and Procedures of Statistics. 1. ed. McGraw-Hill, Londres, $481 \mathrm{pp}$.

TAYLOR, J., 1950. The Comparison of Pairs of Treatments in Split-Plot Experiments. Biometrika, Londres, 37: 443-444.

TAYLOR, W.B., 1957. Incomplete Block Designs with row balanced and recovery of inter-block information. Biometrics, Raleigh, 13:1-12. 